\title{
Active-Constraint Variable Ordering Schemes for Faster Feasibility of Mixed Integer Linear
}

\section{Programs}

by

Jagat Patel, B. Eng.

A thesis submitted to the Faculty of Graduate Studies and Research in partial fulfillment of the requirements for the degree of

Master of Applied Science (M. A. Sc.) in Electrical Engineering

Ottawa-Carleton Institute of Electrical and Computer Engineering Faculty of Engineering

Department of Systems and Computer Engineering

Carleton University

Ottawa, Ontario K1S 5B6, Canada.

September 16, 2002

(C) 2002, Jagat Patel 
National Library

of Canada

Acquisitions and Bibliographic Services

395 Wellington Street Ottawa ON KIA ONA Canada
Bibliothèque nationale

du Canada

Acquisitions et services bibliographiques

395 , rue Welington Ottawa ON KIA ON4 Canada
Your whe Volre raterence

Our the Notre risterence
The author has granted a nonexclusive licence allowing the National Library of Canada to reproduce, loan, distribute or sell copies of this thesis in microform, paper or electronic formats.

The author retains ownership of the copyright in this thesis. Neither the thesis nor substantial extracts from it may be printed or otherwise reproduced without the author's permission.
L'auteur a accordé une licence non exclusive permettant à la Bibliothèque nationale du Canada de reproduire, prêter, distribuer ou vendre des copies de cette thèse sous la forme de microfiche/film, de reproduction sur papier ou sur format électronique.

L'auteur conserve la propriété du droit $d^{\prime}$ 'auteur qui protège cette thèse. Ni la thèse ni des extraits substantiels de celle-ci ne doivent être imprimés ou autrement reproduits sans son autorisation. 
The undersigned hereby recommend to

The Faculty of Graduate Studies and Research

acceptance of the thesis,

\title{
Active-Constraint Variable Ordering Schemes for Faster Feasibility of Mixed Integer Linear Programs
}

\author{
submitted by \\ Jagat Patel,
}

In partial fulfillment of the requirements

for the degree of Master of Applied Science

Show. Chinum

Professor John W. Chinneck, Thesis Supervisor
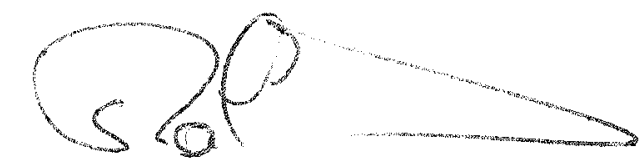

Chairman, Department of Systems and Computer Engineering

Carleton University

September 16, 2002 


\begin{abstract}
Many applications in a wide variety of fields are modeled as Mixed Integer Linear Programming (MILP) problems. Branch and Bound (B\&B) based on LP relaxation and its variations are commonly used to solve general MILPs. In recent years, branching variable selection and node selection schemes have been revisited and several proposed improvements have been shown to be effective. The focus of this thesis is on these two important aspects of the branch and bound algorithm. Dynamic branching variable selection schemes based on the active constraints in the LP relaxation solution are proposed (Active Constraint Schemes). These methods are effective in finding the first feasible solution faster. Different node selection schemes are proposed that are based on the closeness of the B\&B node to integer feasibility. The Depth First Search node selection scheme has better performance compared to these proposed node selection schemes for finding the first integer feasible solution of the MILP problems faster. The times to feasibility of MILPs for the Active Constraint Schemes are compared to those of the CPLEX 6.5 MILP solver [ILOG Corporation 1999c] and OSLMSLV 3.0 [IBM Corporation 1997]).
\end{abstract}




\section{Acknowledgements}

I am very thankful to my thesis supervisor, Prof. John Chinneck (Systems and Computer Eng. Dept., Carleton University) for his constant guidance, encouragement and support in this research. I would like to thank him for answering my never ending questions, patiently listening to my thoughts, believing in me and pointing me in right direction. I would also like to thank him and the department for providing me with the necessary resources needed for this research. I am thankful to Narendra Mehta (Systems and Computer Eng. Dept., Carleton University) for his help on computer resources related problems during this research. I am thankful to ILOG Corporation and IBM Corporation for allowing their MLP solvers to be used for this research. My sincere thanks to Ed Klotz (ILOG Corporation) for his support on CPLEX 6.5 MILP solver [ILOG Corporation 1999c]. Finally I would like to thank my family and friends for their encouragement, support and understanding during my studies. 


\section{A BRIEF OVERVIEW OF BRANCH AND BOUND METHODS}

TO SOLVE MILPS..............................................................................................................

2.1 BRANCH AND BOUND METHOD BASED ON LP RELAXATION SOLUTION …................................

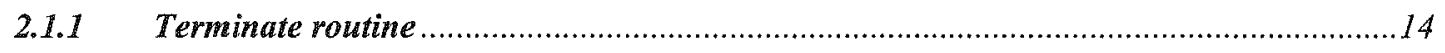

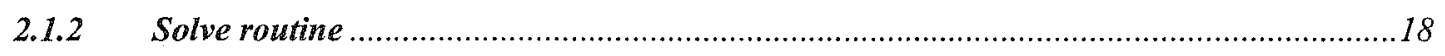

2.1.3 Prune routine and its effect on finding the optimal solution ......................................19

2.1.4 Select routine and its effect on finding the feasible/optimal solution ............................22

2.1.5 Branch routine and its effect on finding the feasible/optimal solution ..........................23

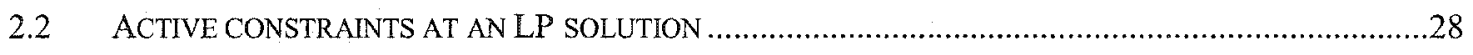

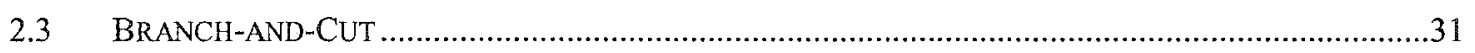

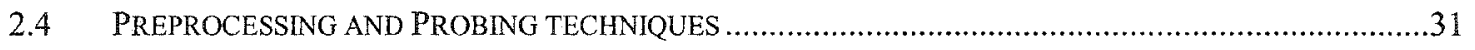

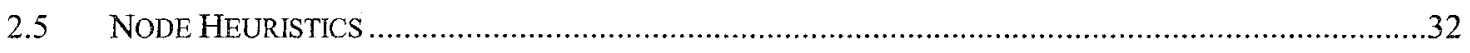




\section{BRANCHING VARIABLE SELECTION METHODS: STATE OF}

THE ART .033

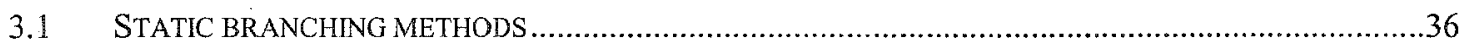

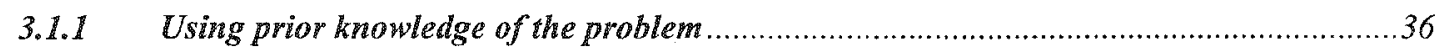

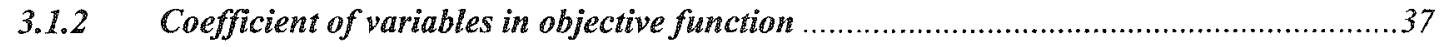

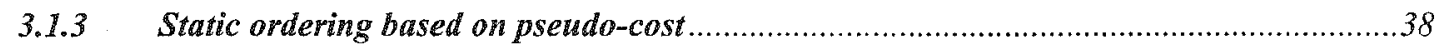

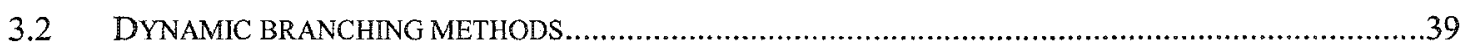

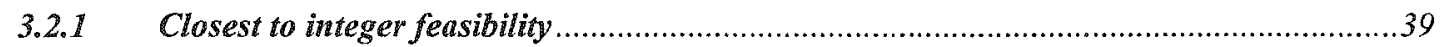

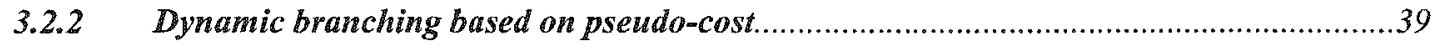

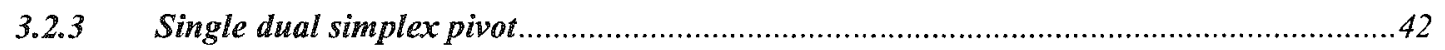

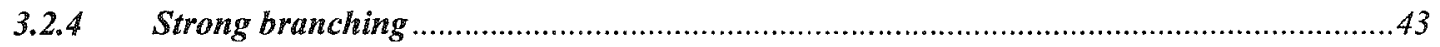

3.2.5 Combination of objective function coefficient and fractional part of integer variable ....43

3.2.6 Combination of estimation and lower bound methods .............................................44

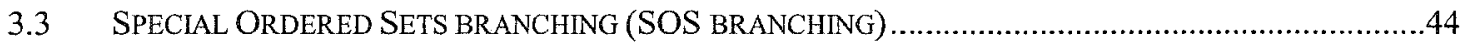

4 NODE SELECTION METHODS: STATE OF THE ART ..................47

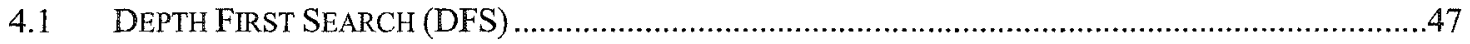

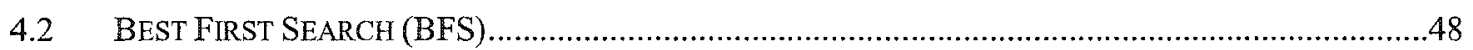

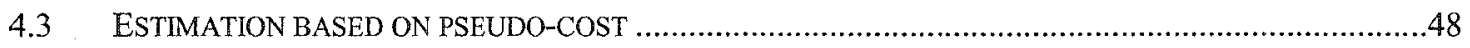

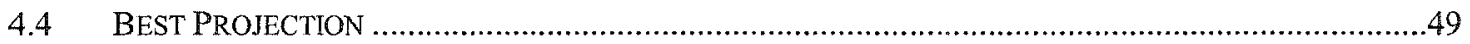

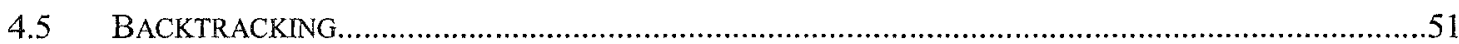

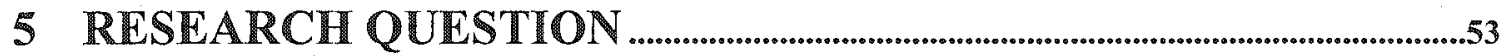

6 DYNAMIC VARIABLE ORDERING SCHEMES BASED ON

ACTIVE CONSTRAINTS............................................................................................................56 
6.1 ACTIVE CONSTRAINT SCHEMES BASED ON THE NUMBER OF ACTIVE CONSTRAINTS CONTAINING THE FRACTIONAL VALUED INTEGER VARIABLE.

6.2 ACTIVE CONSTRAINT SCHEMES BASED ON THE COEFFICIENT OF THE FRACTIONAL VALUED INTEGER VARLABLES IN THE ACTIVE CONSTRAINTS

\section{NODE SELECTION SCHEMES BASED ON INTEGER}

INFEASIBILITIES

7.1 MINIMUM NUMBER OF INTEGER INFEASIBLE VARIABLES (MNIV) .....................................73

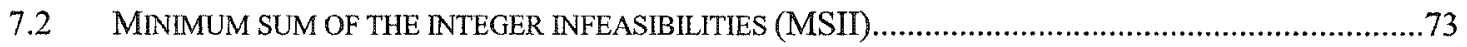

7.3 MINIMUM NUMBER OF CONSTRAINTS CONTAINING INTEGER INFEASIBLE VARIABLES (MNIC) ......74

7.4 MINIMUM RATIO OF NUMBER OF INTEGER INFEASIBLE ACTIVE CONSTRAINT TO TOTAL NUMBER OF

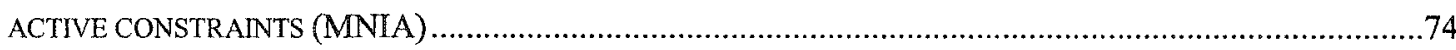

8 EMPIRICAL RESULTS.................................................................................................76

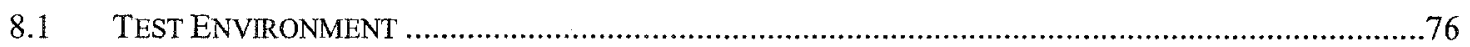

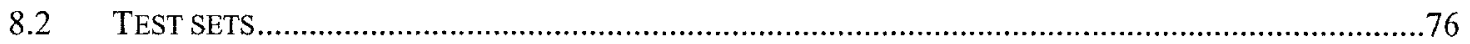

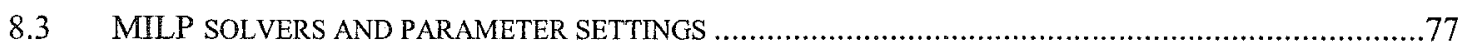

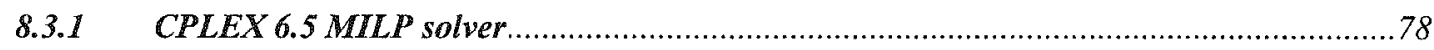

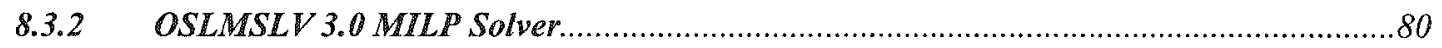

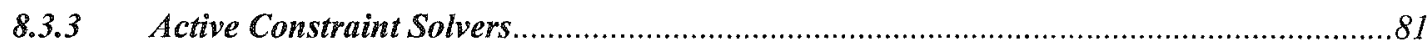

8.3.4 Designation of the Active Constraint Solvers.............................................................82

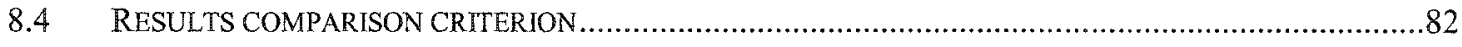

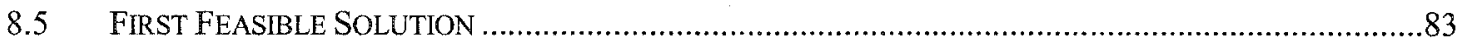

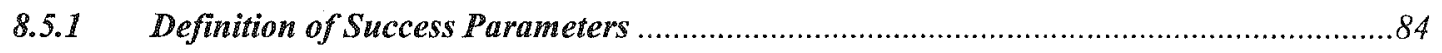

8.5.2 Evaluating the Active Constraint Schemes ....................................................8

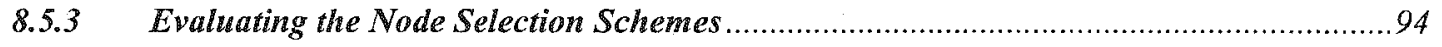


8.5.4 Comparison of Active Constraint Solvers GDFS, CPLEX 6.5 and OSLMSLV 3.0 using

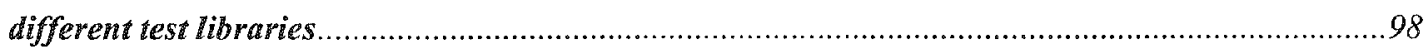

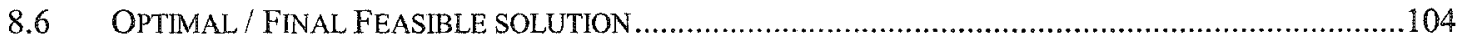

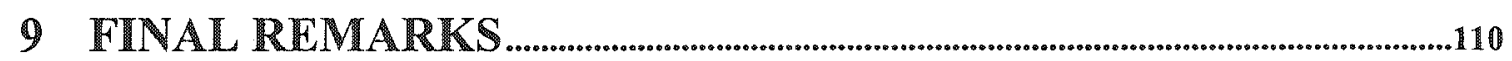

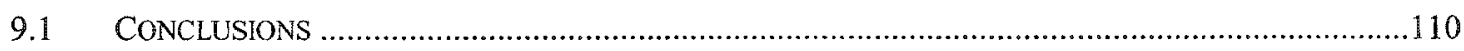

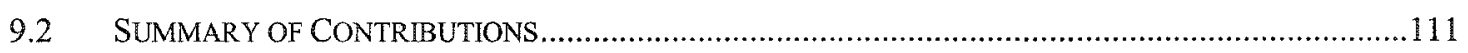

9.3 FUTURE WORK

10 REFERENCES.............................................................................................................116

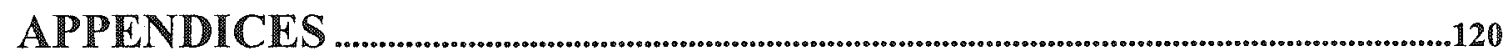

APPENDIX A: THE MILP PROBLEMS OF MIPLIB 3.0, FCTP, MIP BENCHMARKS AND MP TEST MODEL

TEST LIBRARIES

121

APPENDIX B: COMPARISON OF THE ACTIVE CONSTRAINT VARIABLE SELECTION SCHEMES (USING DFS

NODE SELECTION) VS. CPLEX 6.5 FOR FINDING THE FIRST FEASIBLE SOLUTION OF THE MILP PROBLEMS OF

MIPLIB 3.0 LIBRARY

APPENDIX C: COMPARISON OF THE NODE SELECTION SCHEMES (USING ACTIVE CONSTRAINT SCHEME G)

VS. CPLEX 6.5 FOR FINDING THE FIRST FEASIBLE SOLUTION OF THE MILP PROBLEMS OF MIPLIB 3.0

LIBRARY

APPENDIX D: COMPARISON OF CPLEX 6.5, ACTIVE CONSTRAINT SOLVER G/DFS AND OSLMSLV 3.0

MILP SOLVER FOR FINDING THE FIRST FEASIBLE SOLUTION OF MILP PROBLEMS OF MIPLIB 3.0 LIBRARY

APPENDIX E: COMPARISON OF CPLEX 6.5, ACTIVE CONSTRAINT SOLVER G/DFS AND OSLMSLV 3.0 MILP SOLVER FOR FINDING THE FIRST FEASIBLE SOLUTION OF THE MILP PROBLEMS OF FCTP LIBRARY139 APPENDIX F: COMPARISON OF CPLEX 6.5, ACTIVE CONSTRAINT SOLVER G/DFS AND OSLMSLV 3.0 MILP SOLVER FOR FINDING THE FIRST FEASIBLE SOLUTION OF THE MILP PROBLEMS OF THE MIP BENCHMARK UIBRARY 141 
APPENDIX G: COMPARISON OF CPLEX 6.5, ACTIVE CONSTRAINT SOLVER G/DFS AND OSLMSLV 3.0

MILP SOLVER FOR FINDING THE FIRST FEASIBLE SOLUTION OF THE MILP PROBLEMS OF THE MP TEST MODEL LIBRARY

APPENDIX H: COMPARISON OF THE PROPOSED ACTIVE CONSTRAINT SOLVERS IN FINDING THE

OPTIMAL/FINAL SOLUTION OF THE MILP PROBLEMS OF MIPLIB 3.0 LIBRARY 


\section{List of Tables}

Table 1: FSR, FSF, FSF', QSR and QSR' of the Active Constraint Solvers compared individually with CPLEX 6.5 MILP solver over the MILP problems of MIPLIB 3.0 library.

Table 2: FSR, FSF, FSF', QSR and QSR' of Active Constraint Solvers using different node selection schemes compared individually with the CPLEX 6.5 MILP solver over the MILP problems of the MIPLIB 3.0 library.

Table 3: FSR, FSF, FSF', QSR and QSR' of CPLEX 6.5, OSLMSLV 3.0 and Active Constraint Solver G/DFS over the MILP problems of MIPLIB 3.0 library. 100

Table 4: FSR, FSF, FSF', QSR and QSR' of CPLEX 6.5, OSLMSLV 3.0 and Active Constraint Solver G/DFS over the MILP problems of FCTP library. 101

Table 5: FSR, FSF, FSF', QSR and QSR' of CPLEX 6.5, OSLMSLV 3.0 and Active Constraint Solver G/DFS over the MILP problems of MIP Benchmark library. ... 102 Table 6: FSR, FSF, FSF', QSR and QSR' of CPLEX 6.5, OSLMSLV 3.0 and Active Constraint Solver G/DFS over the MILP problems of MP Test Model libraries... 102

Table 7: FSR, FSF, FSF', QSR and QSR' of CPLEX 6.5, OSLMSLV 3.0 and Active Constraint Solver G/DFS over the combined MILP problems of MIPLIB 3.0, FCTP, MIP Benchmark library, MP Test Model libraries 103

Table 8: Designation of the Active Constraint Solvers for finding the optimal solution of the MILP problems 106 
Table 9: OSR of the Active Constraint Solvers compared individually with the CPLEX

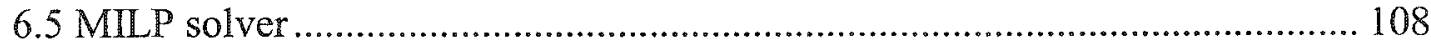




\section{List of Figures}

Figure 1: Difference in the optimal solution of the MLP and its LP relaxation ............. 7

Figure 2: The MILP problem is infeasible but its LP relaxation problem is feasible ...... 8

Figure 3: Solving the MILP problem using B\&B based on the LP relaxation............... 14

Figure 4: Effect of earlier pruning on finding the optimal solution of the MILP........... 21

Figure 5: Solving the MILP problem using Method (a) branching scheme .................. 26

Figure 6: Solving the MILP problem using Method (b) branching scheme................... 27

Figure 7: The active constraints at the comer point optimal solution of the LP ............ 30

Figure 8: The active constraints at the non-corner point optimal solution of the LP ..... 30

Figure 9: Effect on the optimal point of the LP of changing the bounds on a variable... 58

Figure 10: Graphical User Interface (GUI) of the prototype program....................... 149 


\section{Introduction}

There are many real life problems that are modeled as mixed integer linear programming (MILP) problems. Examples include airline crew scheduling [Vance et al. 1997], a global supply chain optimization model [Arntzen et al. 1995], finding fault-tolerant virtual path layouts in an ATM network [Ball and Vakhutinsky 2001] and global routing of nets in VLSI physical design automation [Sherwani 1993].

A MLLP is a type of combinatorial and discrete optimization problem. In a MILP problem, decisions to be made are mapped to the variables. Restrictions (constraints) are in the form of linear equalities and linear inequalities and the goal is to maximize or minimize a linear function of the variables. This is identical to the linear programming (LP) problem [Winston 1995] but the MILP problem requires some or all of the variables to have integer values. This makes MILP problems much more difficult to solve than LP problems. The LP problem obtained by dropping the integer restrictions on variables in a $M \Pi P$ is called the $L P$ relaxation of that MLLP. With recent advances in the computing industry, LP problems with thousands of variables and constraints are considered small and can be easily solved. However it may be difficult to solve MILP problem with just hundreds of variables and constraints [Cornuejols and Dawande 1998].

To prove that an integer program has an objective function value less than a given target is an NP-complete problem [Garey and Johnson 1979]. Following are some commonly used methods for solving MILP problems: 
Branch \& bound based on LP relaxation introduced by Land and Doig [1960] with various improvements discussed by Linderoth and Savelsbergh [1999] and Savelsbergh [1994]. This method is explained in more detail in Section 2.

Cutting plane algorithms introduced by Gomory [1958]. In this method, an LP relaxation of the MILP problem is solved first. If the solution contains fractional values for integer variables then cuts (constraints) are added which remove the LP relaxation optimal point from the feasible region, in such a way that the integer feasible solutions are not affected. The modified problem is resolved and the process is repeated until an optimal solution is found. A detailed discussion of this method is outside the scope of this thesis.

Branch-and-cut algorithm introduced by Padberg and Rinaldi [1987]. This algorithm is a combination of Branch \& bound based on LP relaxation and the cutting plane algorithm. It is explained in more detail in Section 2.

Branch-and-price algorithm, initially discussed by Johnson [1989], is mainly used for solving MILP problems with large numbers of variables. The LP relaxation problem at each node in the B\&B tree is solved using the column generation method [Gilmore and Gomory 1961]. As pointed out by Johnson et al. [2000], conventional integer programming branching may not be effective in the Branch and Price algorithm. Details on this algorithm are outside the scope of this thesis.

Other methods like constraint programming, approximation algorithms and genetic algorithms can be used to solve MLPs. Detailed discussion on these methods is outside the scope of this thesis.

Many MILP solvers that are available today use Branch and Bound (B\&B) based on the LP relaxation or the Branch-and-Cut algorithm (see e.g. CPLEX 6.5 [LOG 
Corporation 1999c], OSLMSLV 3.0 [IBM Corporation 1997]). Researchers have recently revisited $B \& B$ based on $\mathrm{LP}$ relaxation and the Branch-and-Cut algorithm and suggested various improvements to them [Linderoth and Savelsbergh 1999, Johnson et al. 2000, Bixby et al. 2000]. Two important decisions are made in the $B \& B$ process that have a major impact on the computation time and memory required for solving MILP problems. They are the branching variable selection and the node selection. Several methods currently exist for making these decisions and they are discussed in Sections 3 and 4.

The focus of this thesis is on dynamically selecting the branching variable to find the first feasible solution of MILP problems faster. The methods developed can also be used for making branching decision in the Branch-and-Cut algorithm. The branching variable selection methods presented here are based on knowledge of the active constraints at the optimal solution of the LP relaxation problem. Most of the existing branching variable selection schemes are based on objective function measures such as estimated degradation in the objective function value. The methods presented here are independent of the type of problem and hence can be applied to any MILP problem. By presenting the empirical results on different test libraries, it is demonstrated in this thesis that the branching variable selection based on the active constraints in the LP relaxation optimal solution is usually a better approach than the selection scheme used by CPLEX 6.5 and OSLMSLV 3.0 for finding the first feasible solution of MILPs.

Different node selection methods based on closeness to integer feasibility of the B\&B node and the Depth First Search node selection are tried to determine the best combination with the proposed branching variable selection method in finding the first feasible solution of MILP problems. The results achieved using these methods on 
MIPLIB 3.0 library are presented. These results are compared with results obtained using the CPLEX 6.5 MULP solvers. Depth First Search node selection is the best companion of the proposed branching variable selection scheme compared to node selection schemes based on integer infeasibilities for finding the first feasible solution of MLPs. More experimental results are presented to demonstrate that the objective function based branching variable selection schemes are better than the active constraint schemes for finding the optimal solution of MILPs. 


\section{A Brief Overview of Branch and Bound Methods to Solve MILPs}

This section assumes a maximization MLP problem. The minimization MILP problem can be converted to a maximization MLP problem by multiplying its objective function by -1 .

A MLP problem can generally be described as:

Objective: $\quad \operatorname{Max} \mathrm{z}=\mathbf{c x}$

General constraints: $\quad \mathbf{A x}\{\leq, \geq,=\} \mathbf{b}$

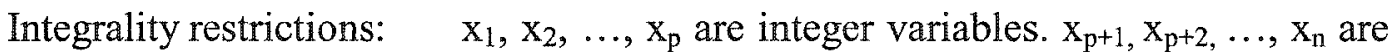
real variables.

Variable bounds: $\quad \mathbb{1} \leq \mathbf{x} \leq \mathbf{u}$

If all of the variables in a MILP problem must have integer values then the problem is called Integer Linear Programming (ILP) or Integer Programming (IP), sometimes referred to as pure integer problems. ILP is a special case of MLP. The term MLP is used in this document to refer to both MILP and ILP. Integrality constraints together with bounding constraints restrict the value of integer variables to either binary values $(0$ or 1$)$ or general integer values $(\ldots,-1,0,1,2, \ldots)$. Any solution that satisfies all constraints (i.e. general constraints, integrality restrictions and variable bounds) is a feasible solution of the MILP (also known as an integer feasible solution).

For a bounded MILP problem, there is a finite number of feasible solutions. The feasible solution with the maximum objective function value is the optimal solution of the MLP problem. An Unbounded MILP problem has feasible solutions with indefinitely 
large objective function values. This usually indicates an error in modeling the problem (e.g. a missing constraint). A MILP problem having no feasible solution is called an infeasible MILP problem. Though the difference between a MILP problem and its LP relaxation problem is only integer value restrictions on some/all of its variables, there can be major differences in their optimal solutions. Consider the following MLP problem.

$$
\begin{aligned}
& \operatorname{Max} z=x+y \\
& -1.2 x+y \leq 0.7 \\
& 10.01 x-y \leq 17 \\
& x, y \text { are integer variables } \\
& x, y \geq 0
\end{aligned}
$$

As shown in Figure 1, the optimal solution of the LP relaxation problem is at point A (2.00,3.11) but the optimal solution of the MLP problem is at point $B(1,1)$. The shaded area in the figure indicates the feasible region of the LP relaxation problem. An isoprofit line of the objective function is shown in the figure as a dotted line. Rounding the value of $\mathrm{x}$ and $\mathrm{y}$ in the LP relaxation optimal solution to the nearest integer value does not yield an optimal solution of the MILP problem, actually it is not even guaranteed to be an integer feasible solution. 


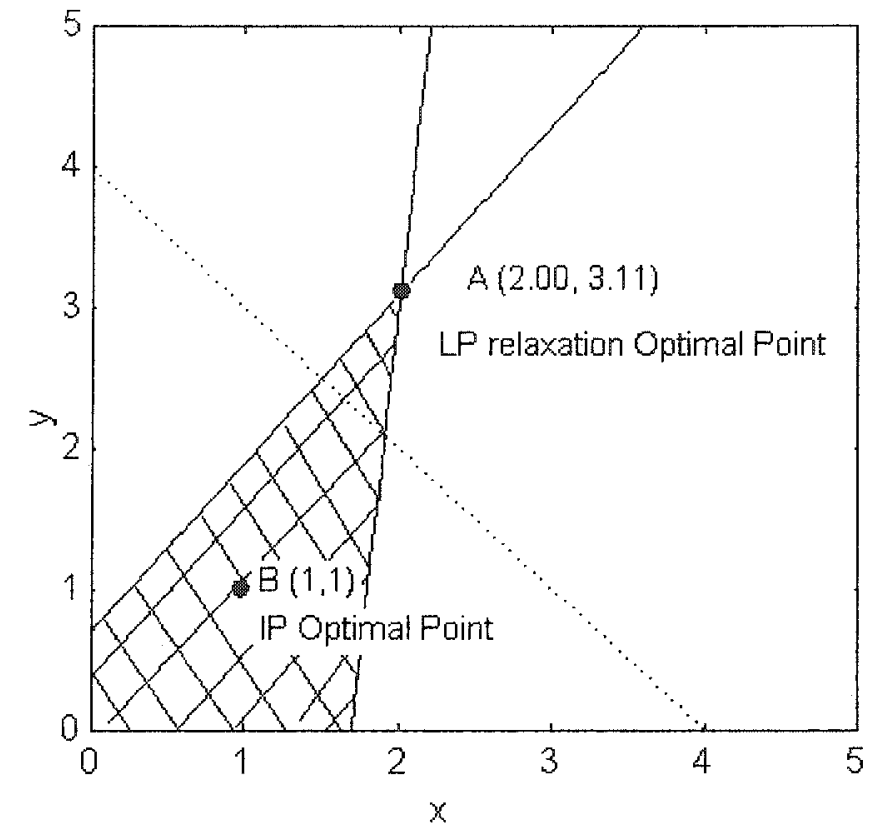

Figure 1: Difference in the optimal solution of the MLP and its LP relaxation

Figure 2 represents the following MILP problem.

$$
\begin{aligned}
& \operatorname{Max} z=x+y \\
& -1.2 x+y \leq 0.7 \\
& 0.9 x+y \leq 5.3 \\
& -3.9 x-y \leq-11 \\
& 11 x-y \leq 27 \\
& x, y \text { are integer variables } \\
& x, y \geq 0
\end{aligned}
$$

This MILP problem has no integer feasible solution but its LP relaxation problem is feasible. 


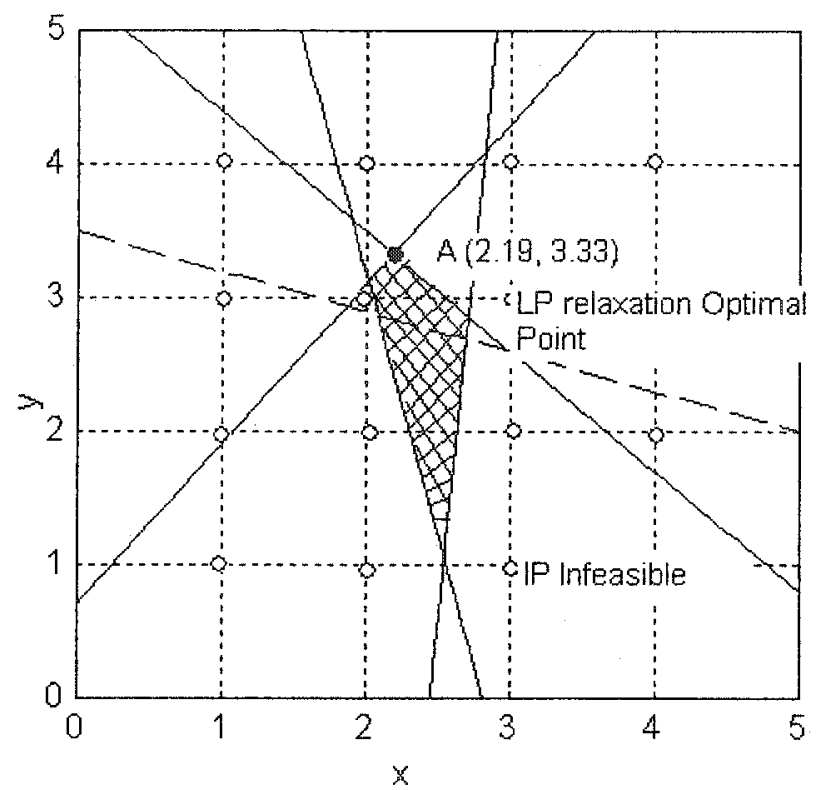

Figure 2: The MILP problem is infeasible but its LP relaxation problem is feasible

\subsection{Branch and Bound method based on $L P$ relaxation solution}

In this method [Land and Doig 1960], a series of LP problems are solved to find the optimal solution of the MILP problem. This creates a Branch and Bound tree with each node in the tree representing an LP problem, which may be either solved or unsolved. The $L P$ relaxation of the original MILP problem is the root node of the B\&B tree. Algorithm 1 outlines the Branch and Bound (B\&B) method based on LP relaxation solutions to find the optimal solution of MILP problems. Initialize, Terminate, Solve, Select, Branch and Prune are routines of the algorithm that are discussed in detail later. 
$N_{i}=$ ith node in $\mathrm{B} \& \mathrm{~B}$ tree. $\mathrm{N}_{0}$ is root node of $\mathrm{B} \& \mathrm{~B}$ tree

Num_Nodes $=$ Total number of nodes in the B\&B tree in addition to the root node

Unsolved_Nodes $=$ Set of unsolved nodes

Current $L P=L P$ relaxation at selected node

Optimal Current $L P=$ Optimal solution of Current_LP. $\phi$ if Current_LP is

infeasible. LP solver sets this after solving Current_LP.

$O b j \_V a l \_C u r r e n t \_L P=\begin{aligned} & \text { Objective function value at Optimal_Current_LP (if } \neq \phi) . L P \\ & \text { solver sets this value after solving Current_LP }\end{aligned}$

$B F S=$ Best known feasible solution of MILP problem being solved.

$L B=$ Objective function value at $B F S$ (i.e. lower bound on solution)

$U B_{i}=$ Objective function value at $L P$ optimal solution of parent node of unsolved node $\mathrm{N}_{\mathrm{i}}$ (i.e. upper bound on solution)

\section{Step 0: Initialize}

$$
\begin{aligned}
& \text { Unsolved_Nodes }=\left\{N_{0}\right\} \\
& \text { Num_Nodes }=0 \\
& \text { Current_LP }=\phi \\
& \text { BFS }=\phi \\
& L B=-\infty \\
& U B_{0}=\infty
\end{aligned}
$$

\section{Step 1: Terminate?}

IF (Unsolved_Nodes $=\phi)$ THEN

\section{ENDIF}

Exit with optimum solution $=B F S$ and optimum objective function value $=L B$

Step 2: Select:

Select a node, say $N_{i}$, from Unsolved_Nodes

Unsolved_Nodes $=$ Unsolved_Nodes $\backslash N_{i}$

Current_LP $=$ LP at node $N_{i}$

Step 3: Solve:

Solve Current $L P$ using any method for solving linear programs (e.g. simplex method). IF (Optimal_Current_LP $=\phi \mathrm{OR}$

$$
\left.\left(L B \geq O b j \_V a I+C u r r e n t \_L P\right)\right) \text { THEN }
$$

GOTO Terminate? 
ELSE

IF $\left(\mathrm{x}_{1}, \mathrm{x}_{2}, \ldots, \mathrm{x}_{\mathrm{p}}\right.$ have integer values in Optimal_Current_LP $)$ THEN $B F S=$ Optimal_Current_LP $L B=O b j \_$Val_Current_LP GOTO Prune

ELSE

GOTO Branch

ENDIF

ENDIF

Step 4: Branch

Select an integer variable $\mathrm{x}_{\mathrm{j}}$ having non-integer value $\mathrm{v}_{\mathrm{j}}$ in Optimal_Current_LP.

Create two child nodes, $\mathrm{N}_{\mathrm{Num}_{-} \text {Nodest } 1}$ and $\mathrm{N}_{\text {Num_Nodes }+2}$, of $\mathrm{N}_{\mathrm{i}}$.

$L P$ at node $\mathrm{N}_{\text {Num_Nodes+1 }+1}=$ Current_LP $\angle$ Constraint $\mathrm{x}_{\mathrm{j}} \leq\left\lfloor\mathrm{v}_{\mathrm{j}}\right\rfloor$

$\mathrm{LP}$ at node $\mathrm{N}_{\mathrm{Num} \_ \text {Nodes }+2}=$ Current_LP $\cup$ constraint $\mathrm{x}_{\mathrm{j}} \geq\left\lceil\mathrm{v}_{\mathrm{j}}\right\rceil$

$\mathrm{UB}_{\text {Num_Nodes+1 }}=O b j \_$Val_Current_LP

$\mathrm{UB}_{\text {Num_Nodes }+2}=$ Obj_Val_Current_LP

Unsolved_Nodes $=$ Unsolved_Nodes $\cup\left\{\mathrm{N}_{\text {Num_Nodes }+1}\right\} \cup\left\{\mathrm{N}_{\text {Num_Nodes }+2}\right\}$

Num_Nodes $=$ Num_Nodes +2

GOTO Terminate?.

\section{Step 5: Prune}

FOR each node $N_{k}$ in Unsolved_Nodes

$$
\begin{aligned}
& \text { IF }\left(L B \geq U B_{k}\right) \text { THEN } \\
& \text { Unsolved_Nodes }=\text { Unsolved_Nodes } \backslash N_{k} \\
& \text { ENDIF }
\end{aligned}
$$

END FOR

GOTO Terminate?.

\section{Algorithm 1: Simple Branch and Bound based on LP Relaxation for Maximization}


Consider the following MLP for example. The solution of the LP relaxation problem at each node of the B\&B tree is shown with the help of Figure 3.

$$
\begin{aligned}
& \operatorname{Max} z=0.125 x+y \\
& -x+y \leq 2 \\
& 2.5 x+y \leq 14.5 \\
& x, y \text { are integer variables } \\
& x, y \geq 0
\end{aligned}
$$

The LP relaxation of the original MILP problem is the root node of the $B \& B$ tree (Node 0 in Figure 3 (a)). The optimal point of the LP relaxation problem at Node 0 is $(3.57,5.57)$. Since the solution is not integer feasible, the Branch routine selects an integer variable ( $\mathrm{x}$ in this case) having non-integer value in the optimal solution as a branching variable to create two new child nodes - Node 1 and Node 2. The upper bound on these two nodes is set to the objective function value at the optimal solution of Node 0 , i.e. 8.78. A bounding constraint of $x \leq 3$ is added to the LP relaxation problem at Node 1 (Figure 3 (b)), and the bounding constraint of $x \geq 4$ is added to the LP relaxation problem at Node 2 (Figure 3 (c)).

The Select routine chooses one of these two nodes, say Node 1 , as the next node to solve, and the LP relaxation problem at the selected node is solved by the Solve routine. The optimal point of the LP relaxation problem at Node 1 is $(3.0,5.0)$ which is also integer feasible. Since this is the first feasible solution, the lower bound is set to the objective function value of the LP optimal solution (i.e. 7.7), and the Prune routine is called. The Prune routine compares the upper bounds on the unsolved nodes, currently only Node 2, with the new lower bound value. Since the upper bound on Node 2 is 
greater than the new lower bound value, there is a chance of finding a better integer feasible solution in Node 2 or its descendents, and so Node 2 is not pruned.

Node 2 is solved next and variable y is branched on to create Node 3 (Figure 3 (d)) and Node 4 (Figure 3 (e)). The LP problem at Node 3 has an optimal solution at (4.2, 4.0) and variable $x$ is branched on to create Node 5 (Figure $3(f)$ ) and Node 6 (Figure 3 (g)). The LP problem at Node 4 is infeasible and so is ignored. The LP problem at Node 5 has an optimal solution at $(4.0,4.0)$ which is also integer feasible. The Objective function value at its LP optimal solution (i.e. 7.6) is less than the current lower bound and so it does not change the lower bound value. Node 6 has an LP objective function value that is less than the current lower bound and so this node is not considered further (i.e. no child nodes are added). The set of unsolved nodes is now empty. Hence the LP relaxation solution at Node 1 is the optimal solution of the MILP, and the objective function value at the optimal solution is 7.7 . 


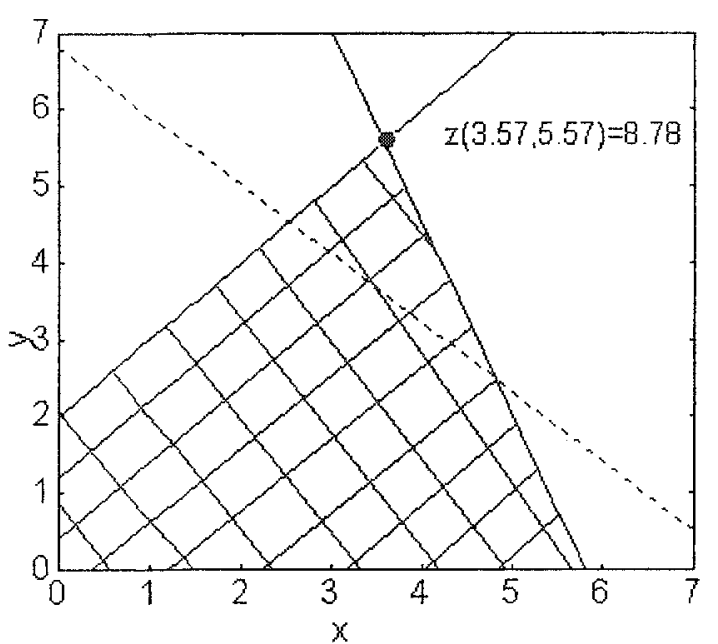

(a) Node 0

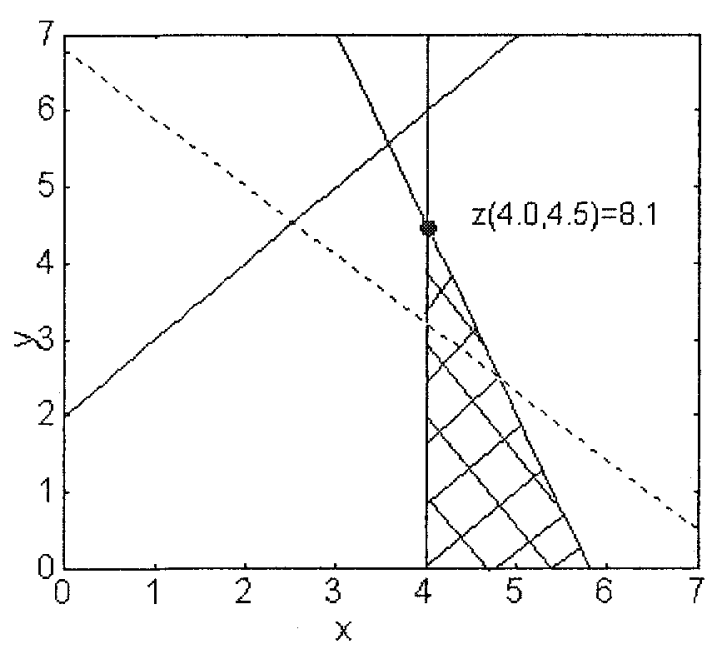

(c) Node 2

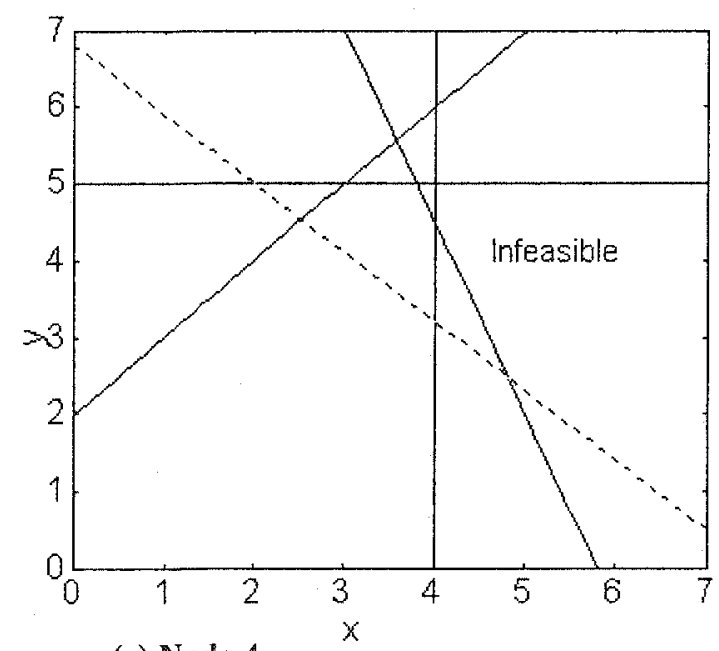

(e) Node 4

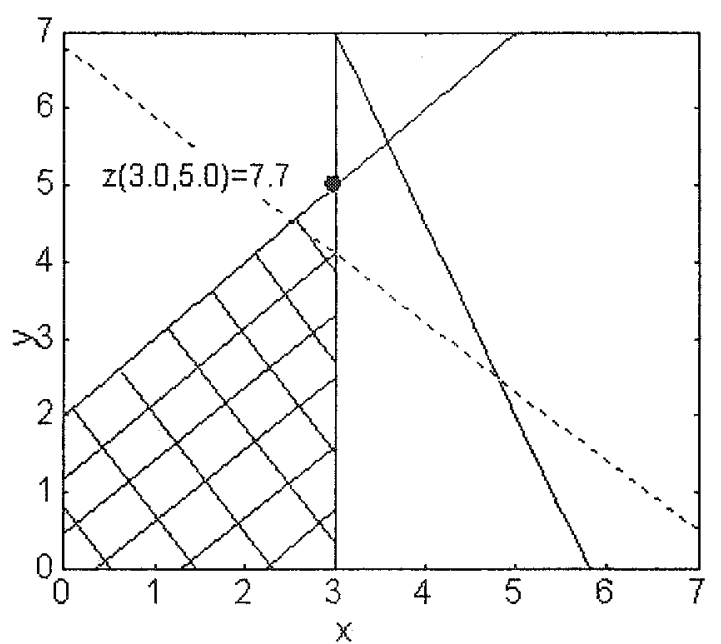

(b) Node 1

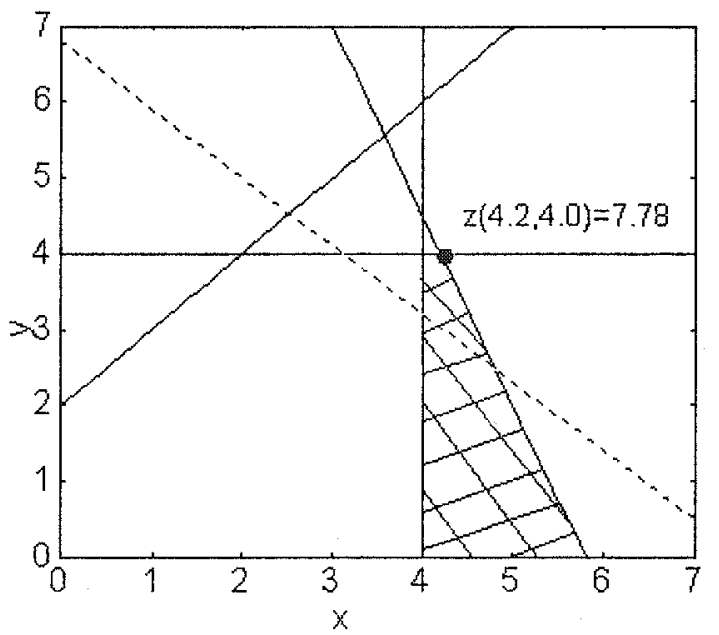

(d) Node 3

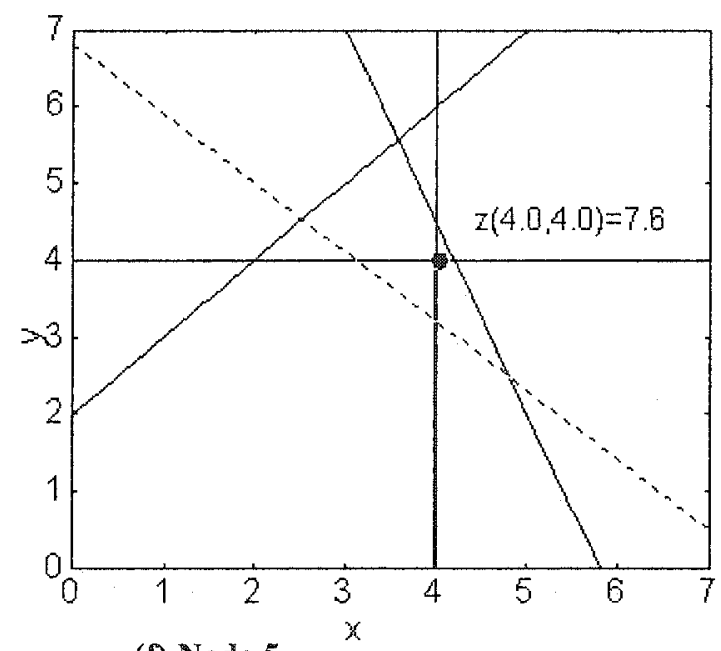

(f) Node 5 


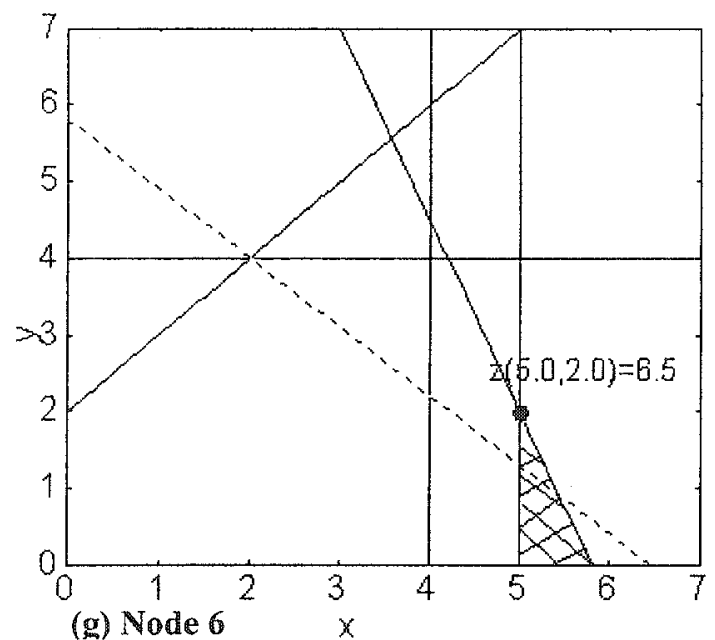

Figure 3: Solving the MILP problem using B\&B based on the $L P$ relaxation

\subsubsection{Terminate routine}

The Terminate routine checks the following conditions:

\section{- Optimality condition}

The Feasible region of the LP relaxation problem at the root node is a super-set of the feasible region of the original MILP. In the branch and bound method, only the Branch and Prune routines change the feasible region of the original LP relaxation problem. The Branch routine does not change the feasible region of the original MILP. The Prune routine removes part of the feasible region of the original MILP that is guaranteed to not provide an integer feasible solution that is better than the best known integer feasible solution (BFS). If the $B F S$ is non-empty when the entire $\mathrm{B} \& \mathrm{~B}$ tree has been explored (i.e. Unsolved_Nodes is empty) then no other integer feasible solution exists that is better than BFS. BFS is the optimal solution of the MILP. 


\section{- Infeasibility condition}

The foregoing explanation of the optimality condition also applies here. If $B F S$ is empty when the entire $\mathrm{B} \& \mathrm{~B}$ tree has been explored (i.e. Unsolved_Nodes list is empty) then no feasible solution exists for the original MILP. The MLP problem is infeasible.

\section{- User specified limits}

Sometimes it is very hard or even not possible with limited computer resources (i.e. memory and cpu speed) to find the optimal solution of a MILP that has hundreds of variables and constraints. On the other hand, it might be easy to find the optimal solution of a MLP with thousands of variables and constraints. Given a MLP problem, it is not possible to predict in advance the amount of effort required for solving it. Under this uncertainty, the user would not like to wait indefinitely to find the optimal solution. Sometimes the optimal solution is found earlier in the B\&B process, but a large amount of effort is spent in proving optimality, i.e. exploring unsolved nodes to prove that they do not have an integer feasible solution that is better than the known integer feasible solution. The user can set limits on the following parameters, and the Terminate routine can check their values to decide if the algorithm has to be terminated (not shown in Algorithm 1). The modern MILP solvers (e.g. CPLEX 6.5, OSLMSLV 3.0) allow specifying the values for most of these limits.

- Time limit: Maximum amount of real time (or cpu time) in seconds for which $B \& B$ search can be continued. 
- Memory limit: Computers have limited amount of run-time memory for holding programs and their data when they are being executed. In the absence of virtual memory handling, program execution can abnormally terminate when there is no free run-time memory. The $\mathrm{B} \& \mathrm{~B}$ method requires memory for holding $\mathrm{B} \& \mathrm{~B}$ tree data. The B\&B tree data keeps on growing as the B\&B method continues. To avoid abnormal termination, the user can set the memory limit to a value less than the amount of available memory. When this limit is reached, the B\&B method terminates normally. Most modern operating systems use a method called paging in which run-time memory is freed-up by storing data on disk, loading it back into memory when needed. This is also called memory swapping. Memory swapping slows down overall execution time. Some solvers themselves perform similar memory swapping. They can minimize the amount of swapping by using their knowledge of the $B \& B$ tree data. When the user specified memory limit is reached, the $B \& B$ solver begins storing tree data on disk. The user can also specify a limit on disk data size.

- Optimality tolerance: The algorithm is terminated when the percentage difference between the objective function value of the best known feasible solution and the optimal solution of the MILP problem is no more than the optimality tolerance. For example, the user can set the optimality tolerance to $1 \%$ and it means that any feasible solution whose objective function value differs from the optimal solution objective function value by $1 \%$ (or less) is an acceptable final solution. To calculate the difference, the best upper bound on an unsolved child node is used as the estimated optimal solution objective function value. If the algorithm is 
terminated when the difference is equal to $a \%$ ( $a \leq$ optimality tolerance), then the actual difference between the objective function value of the final solution and the optimal solution must be less than $a \%$, or the final solution might even be optimal solution, however this cannot be proved without further expansion of the $B \& B$ tree.

- Number of feasible solutions: For some types of MILP problems only a feasible solution is required and there is no need for the optimal solution. In such cases, this parameter can be set to 1 . The user might be satisfied with the quality of the best feasible solution that is available after finding a certain number of feasible solutions in which case he/she can set this parameter to assure some degree of quality.

- Number of solved B\&B nodes: This is an indirect way of specifying a limit on computation time and memory. The number of solved $B \& B$ nodes indicates how many LP relaxation problems are solved. It also gives an estimate of the amount of memory used to store the B\&B tree data which represents the major portion of the overall memory consumption of the algorithm.

- Total number of simplex iterations: A limit on this parameter can be set only when the simplex method is used to solve the LP relaxation problems. It is the sum of the number of simplex iterations used to solve the LP relaxation problems. It is an indirect way of specifying a limit on computation time.

If the algorithm terminates without finding the optimal solution then the quality of the best known integer feasible solution is measured using its optimality gap: 


$$
\text { optimality gap }=\frac{a b s(L B-\max (U B))}{a b s(L B)}
$$

In the above equation the term $\max (\mathrm{UB})$ represents the maximum upper bound on the unsolved nodes.

\subsubsection{Solve routine}

This routine is used to solve the LP relaxation at each node of the B\&B tree. A faster algorithm for solving LP problems reduces the overall computation time to solve the MILP problem. There are two methods commonly used for solving LP problems:

\section{- Simplex Algorithm}

The Simplex algorithm introduced by Dantzig [1949] progressively improves the objective function value by iterating through corner points of the feasible region. Corner points are the boundary points of the feasible region where two or more constraints intersect.

\section{- Interior-point Algorithms}

This method is generally used to solve large LPs (see e.g. Karmarkar [1984]). Discussion of this method is outside the scope of this thesis.

There are other techniques for solving LPs e.g. network simplex for solving transportation, transshipment, assignment and other type of minimum-cost network flow problems. Discussion on these techniques is outside the scope of this thesis. Winston [1995] has more information on other techniques.

As mentioned by Johnson et al. [2000], the simplex algorithm is commonly used for solving LP problems in the B\&B method. In a B\&B tree, the LP problem at a child 
node differs from the LP problem at its parent node by a single bounding constraint on the branching variable. The Dual Simplex method (a variation of simplex method - see e.g. [Winston 1995]) can use the parent node optimal tableau to quickly reach an optimal solution of the child node. Usually this requires only a few simplex iterations to find the optimal solution of the child node. The simplex algorithm is a better choice for solving small to medium sized LPs than interior point algorithms.

\subsubsection{Prune routine and its effect on finding the optimal solution}

The Prune routine is called when the first integer feasible solution is found and each time an integer feasible solution with objective function value better than the current lower bound is found. The lower bound $(L B)$ is set to the objective function value of the new integer feasible solution before calling the Prune routine. The Prune routine removes nodes from the set of unsolved nodes whose upper bound is equal to or worse than the $L B$. Recall that the upper bounds on the unsolved nodes are set to the objective function value of their parent node's LP relaxation optimal solution. It is safe to consider the parent node's LP relaxation solution for the comparison because the parent node feasible region is a superset of the feasible region of each of its child nodes. The integer feasible solution that can be obtained from an unsolved node or any of its descendents cannot be better than its upper bound.

Pruning reduces the search space that has to be explored to find the optimal solution of the MILP problem. The LP relaxation problems at nodes higher in the $B \& B$ tree generally represent a larger feasible region than the LP relaxation problems at nodes closer to the bottom of the B\&B tree. The search space to be explored is larger at nodes 
closer to the top of the B\&B tree compared to ones closer to the bottom. The earlier an integer feasible solution is found, the higher the chances of pruning unsolved nodes closer to the top of the B\&B tree, which greatly reduces the search space to be explored. Reducing the unexplored search space will improve the chances of finding the optimal solution faster. The following example shows how earlier pruning helps in finding the optimal solution faster.

$$
\begin{aligned}
& \text { Max } z=x+3 y \\
& -3 x+5 y \leq 8 \\
& 5 x+4 y \leq 39 \\
& x, y \text { are integer variables } \\
& x, y \geq 0
\end{aligned}
$$

Figure 4 shows two possible ways of solving this MILP problem. Square boxes in the figure represent nodes of the $B \& B$ tree, and values inside them represent the optimal solution of the LP relaxation at that node. The lines connecting the nodes are labeled with the bounding constraint that is added during branching. The node numbers inside the boxes represent the order in which the nodes are solved. Both Method (a) and Method (b) perform node selection in Depth First Search (DFS) manner. 
Method (a)
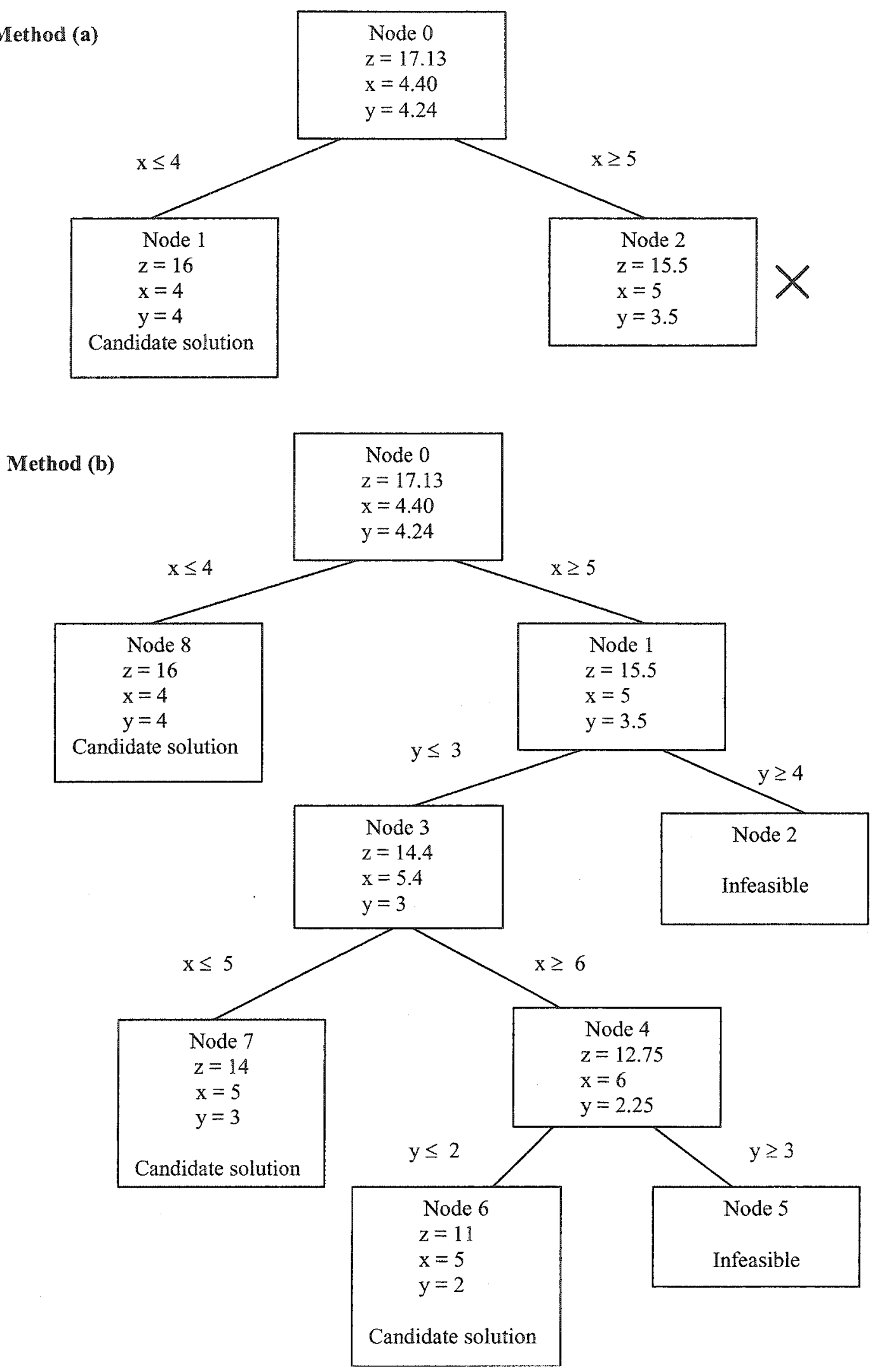

Figure 4: Effect of earlier pruning on finding the optimal solution of the MILP 
Method (a) favors the lower branch (i.e. the branch that sets the upper bound on the branching variable) and Method (b) favors the upper branch (i.e. the branch that sets the lower bound on the branching variable). After solving the root node LP relaxation problem, Method (a) selects the node along the $x \leq 4$ branch as the next node to solve while Method (b) selects the node along the $x \geq 5$ branch. Method (a) finds the first integer feasible solution at Node 1 . Node 2 has an objective function value less than the lower bound and so it is pruned. This is shown by a cross-mark $(X)$ close to that node in the figure. Hence Method (a) solves 3 LP problems to find the optimal solution of the MILP problem.

Method (b) solves 9 LP problems to find the optimal solution of the MILP problem. It found the first integer feasible solution at Node 6 which did not help to prune any nodes. Finding the first integer feasible solution faster and pruning the nodes closer to the top of the $B \& B$ tree, as done by Method (a), has a higher impact on reducing the search space. As a result of earlier pruning, Method (a) was able to find the optimal solution faster than Method (b).

\subsubsection{Select routine and its effect on finding the feasible/optimal solution}

This routine selects the unsolved node to be solved next. As mentioned by Johnson et al. [2000] and Linderoth and Savelsbergh [1999], the major criterion in this selection is the

probability of finding a good integer feasible solution from that node. Finding a good integer feasible solution faster might improve the lower bound and trigger pruning. Pruning unsolved nodes earlier in the $B \& B$ process has advantages as described in Section 2.1.3. It is also useful when it is the only integer feasible solution available when 
a user specified limit (e.g. time limit) is reached. For some types of MLP problems, only an integer feasible solution is needed. Some of the existing node selection strategies are explained in Section 4.

\subsubsection{Branch routine and its effect on finding the feasible/optimal solution}

The Branch routine divides the feasible region of the LP relaxation at the current node (Current_LP) into two or more child nodes such that:

- The integer feasible region of the parent node LP problem is the same as the combined integer feasible regions of its child node LP problems.

- An optimal solution of the parent node LP problem is not a feasible solution in any of its child node LP problems.

As shown in Algorithm 1, usually only two child nodes are created to divide the feasible region of Current_LP. An integer variable having non-integral value in the optimal solution of Current_LP is selected as the branching variable. The integer variables having non-integer values at the LP relaxation optimal solution are referred to as "fractional valued integer variables" or "integer infeasible variables". In this thesis both terminologies are used. The LP relaxation problem at the child node is Current_LP plus a revised bound on the branching variable. The revised bounds on the branching variable divide the feasible region of the parent node LP relaxation and satisfy the above two conditions. Solving the child node LP relaxation leads to one of the following three conditions:

(a) Either the child node LP relaxation is infeasible or its optimal objective function value is not better than the current lower bound (if any). No new nodes are added to 
the set of unsolved nodes and the search is terminated for this branch. Reaching this condition faster reduces the overall search space and helps in finding the optimal solution faster.

(b) The optimal solution of the child node LP relaxation is integer feasible. The pruning condition is checked to see if a new lower bound is found in which case the Prune routine is called. Reaching this condition faster finds an integer feasible solution faster and causes earlier pruning in the B\&B process. Earlier pruning has advantages as described in Section 2.1.3. Finding an integer feasible solution faster has advantages as described in Section 2.1.4.

(c) The optimal solution of the child node LP relaxation is found which is not integer feasible and its objective function value is better than the current lower bound (if any). The feasible region of this child node is divided using the Branch routine and new nodes are added to the set of unsolved nodes. The upper bound on these new nodes is set to the objective function value at the LP relaxation optimal solution of the child node.

Thus the Branch routine can play an important role in:

- Decreasing the upper bounds on new child nodes. This will increase the chances of pruning those nodes and will help in finding the optimal solution faster.

- Effectively eliminating the non-integer feasible solutions from the LP feasible region. This can lead to either finding an integer feasible solution or declaring that no feasible solution exists. This is useful for finding the first integer feasible solution faster. 
The following example shows how selection of the branching variable in the Branch routine affects the number of $B \& B$ nodes that are solved to find the first integer feasible solution and the optimal solution of the MILP problem.

$$
\begin{aligned}
& \text { Max } z=x+y \\
& -5.5 x+5 y \leq 7.5 \\
& 5 x+2 y \leq 15 \\
& -2 x-4.5 y \leq-11 \\
& x, y \text { are integer variables } \\
& x, y \geq 0
\end{aligned}
$$

Two methods using different branching variable selection schemes are used to solve this problem. Pruning is not done while solving this problem in order to ignore the effect of node selection on the B\&B tree. After solving the root node LP relaxation problem, Method (a) selects variable $\mathrm{x}$ as the branching variable and Method (b) selects variable $y$.

As shown in Figure 5, Method (a) solves 9 LP problems to find the optimal solution of the MILP problem. If the root node is considered as depth 0 of the $B \& B$ tree then the first integer feasible solution is obtained at depth 2 .

As shown in Figure 6, Method (b) solves $11 \mathrm{LP}$ problems to find the optimal solution of the same MILP problem and the first integer feasible solution is found at depth 3 of the $B \& B$ tree. The branching variable selection scheme affects the number of $\mathrm{B} \& \mathrm{~B}$ nodes solved to find the first integer feasible solution and the optimal solution of the MILP problem. 


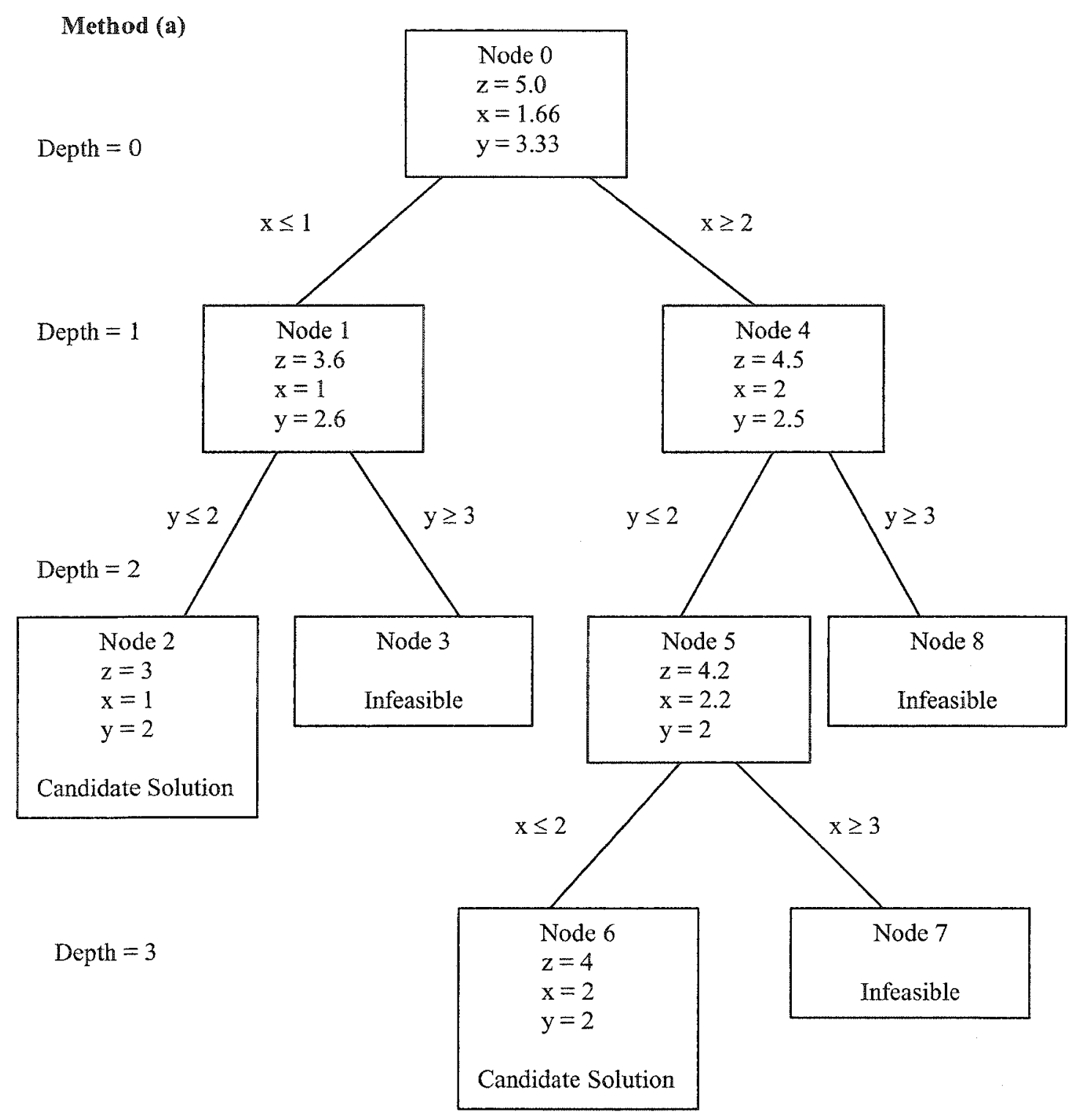

Figure 5: Solving the MILP problem using Method (a) branching scheme 


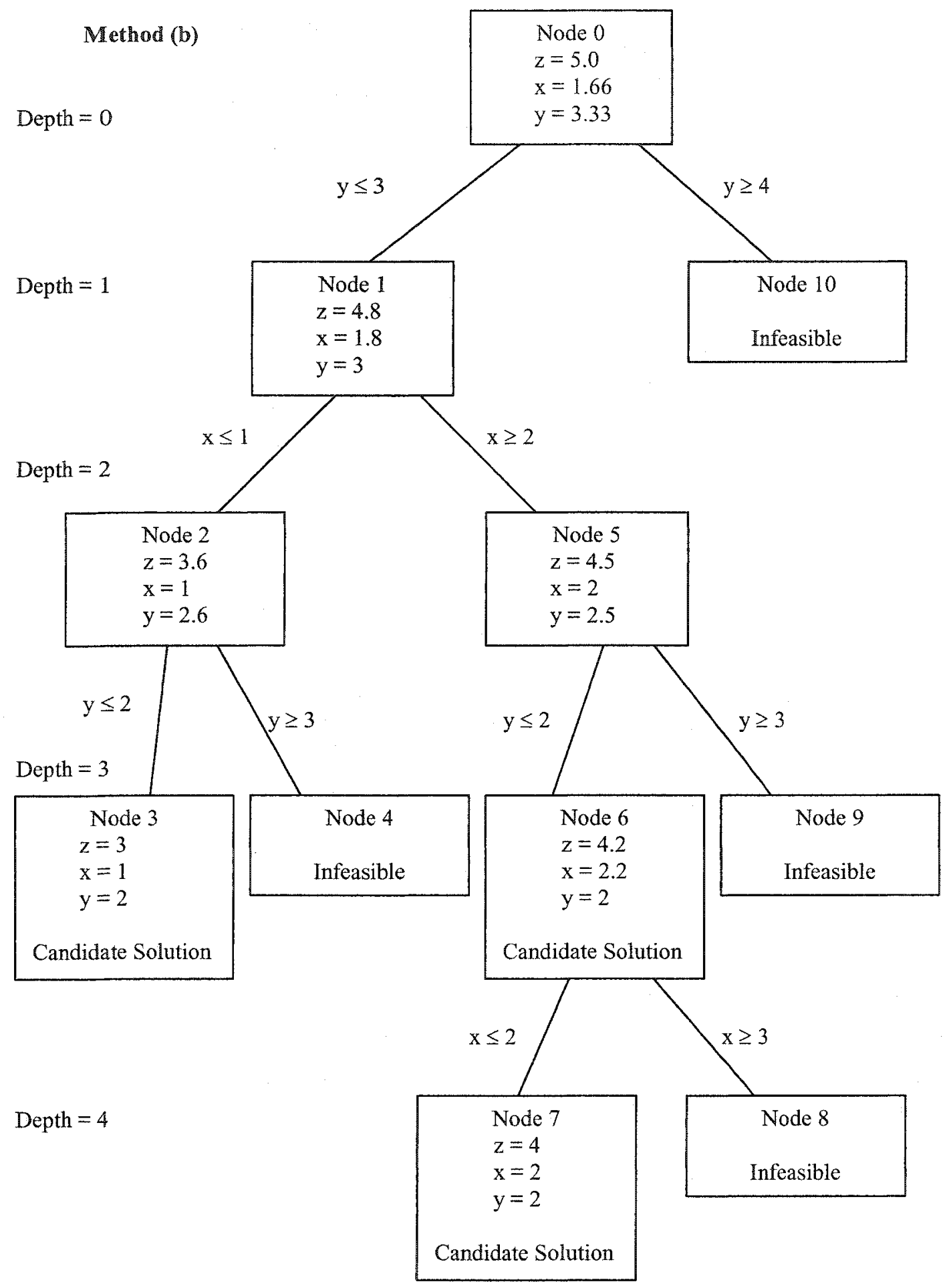

Figure 6: Solving the MILP problem using Method (b) branching scheme 
Existing methods for implementing the Branch routine are discussed in Section 3. Most of these methods select the fractional valued integer variable that gives the largest estimated degradation in the objective function value at the optimal solution of the child node LP relaxation. This decreases the upper bound value of the $B \& B$ tree. As explained before, if the Branch and Select routines can help in finding the first integer feasible solution faster then they can provide a lower bound earlier and prune nodes closer to the top of the B\&B tree. As explained in Section 2.1.4, the Select routine attempts to improve the lower bound throughout the B\&B process. An optimal solution is found when the lower bound and the upper bound of the $\mathrm{B} \& \mathrm{~B}$ tree converge. For a given MLP, faster convergence of the lower bound and the upper bound of its $B \& B$ tree leads to finding the optimal solution faster. The Select and Branch routines impact the number of branch and bound nodes that are solved to find the optimal solution of the MILP problem.

\subsection{Active constraints at an LP solution}

Let us consider the "active constraints" [Winston 1995] or the "binding constraints" and their relationship to the optimal solution of the LP problem. If an optimal solution of the LP problem exists then it has at least one corner point (see Section 2.1.2 for explanation on corner point) that is optimal. At a corner point optimal solution, two or more constraints intersecting at that point are active. Figure 7 shows the corner point optimal solution (Point $\mathrm{A}$ in the figure) of the following LP problem. The active constraints are shown by dark lines in the figure. 


$$
\begin{aligned}
& \operatorname{Max} z=x+y \\
& -0.3 x+y \leq 1 \\
& 3 x+y \leq 11 \\
& -1.2 x+y \leq-1 \\
& -11 x+y \leq 20 \\
& x, y \geq 0
\end{aligned}
$$

If there is more than one optimal solution (alternate optimal solutions) of the LP problem then all points on the segment of some constraint boundary are optimal. For the 2-dimension problem, it would be all points on a line segment of the constraint boundary line. The endpoints of this line segment are the corner point optimal solutions. In the previous LP problem if the objective function is $3 \mathrm{x}+\mathrm{y}$ then, as shown in Figure 8 , all points on the line segment $A B$, including the points $A$ and $B$, are optimal points. All optimal solutions except the ones at the points $\mathrm{A}$ and $\mathrm{B}$ have a single active constraint which is shown by a dark line in the figure. 


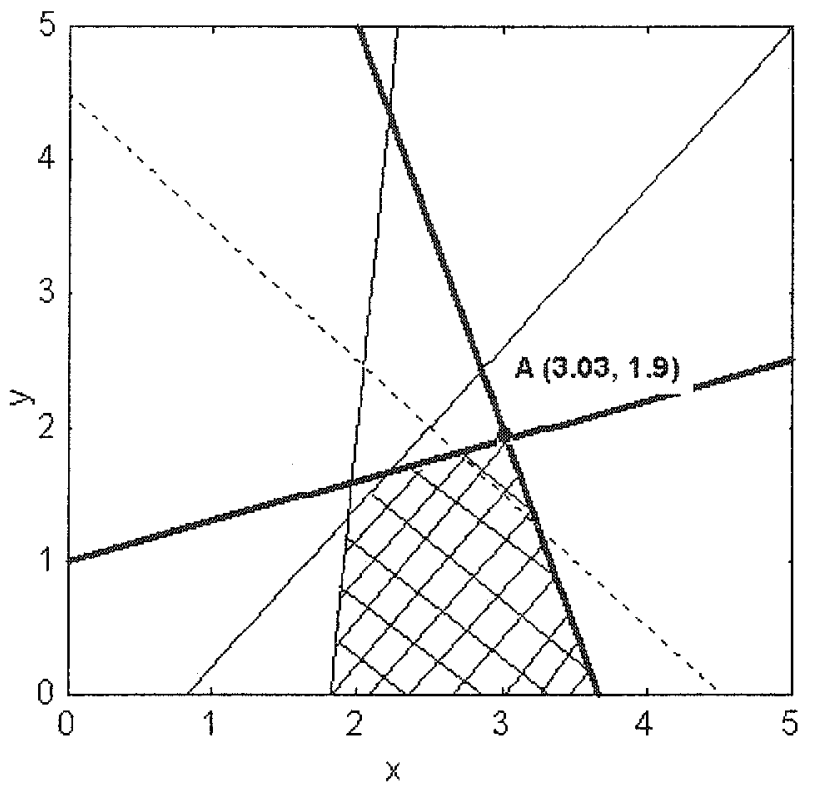

Figure 7: The active constraints at the comer point optimal solution of the $L P$

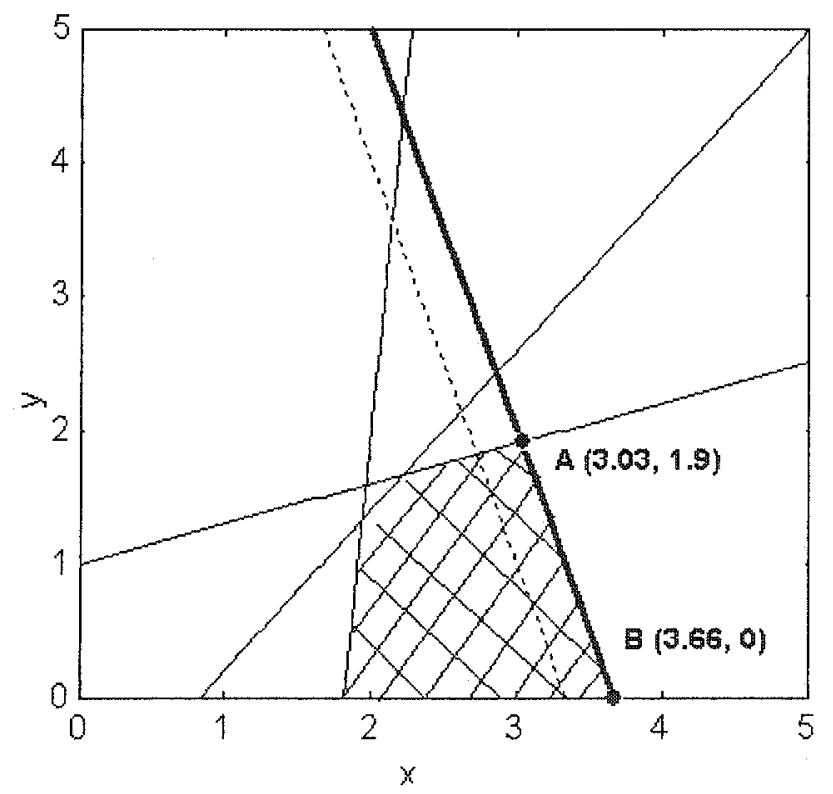

Figure 8: The active constraints at the non-corner point optimal solution of the $L \mathbb{P}$ 


\subsection{Branch-and-Cut}

The Branch and Cut algorithm [Padberg and Rinaldi 1987] first solves the LP relaxation of the original MILP problem. If the optimal solution contains fractional valued integer variables, then cuts (constraints) are added and the problem is resolved as is done in the Cutting Plane algorithm (see Section 1). The process of solving the LP relaxation problem and adding the cuts is continued until either the problem becomes infeasible or no more cuts can be added. In the latter case, child nodes are added by branching on a fractional valued integer variable. The $B \& B$ procedure continues by selecting a node, solving its LP relaxation problem and adding cuts again. The CPLEX 6.5 MILP solver and the OSLMSLV 3.0 MLP solver support cut generation during the B\&B process.

\subsection{Preprocessing and Probing techniques}

The overall effect of basic preprocessing and probing techniques is to improve the representation of the MILP problem and its LP relaxation such that the time/effort spent to solve the LP relaxation problem at any node in the B\&B tree and to reach the final solution of the MILP problem is reduced. The basic preprocessing technique checks whether the MLP problem is infeasible or contains redundant constraints. It also tightens the variable bounds and performs constraint coefficient reduction in order to reduce the solution time of the LP relaxation problem and the MILP problem as a whole. The basic probing technique evaluates the consequences of fixing a binary variable to either of its bounds by examining the resulting constraints. This technique tries to fix the binary variable's value and tighten its coefficient in the constraints. A detailed discussion on the advanced preprocessing and probing techniques is outside the scope of this thesis. 
Savelsbergh [1994] discuss various preprocessing and probing techniques. Preprocessing and probing techniques are available in commercial MILP solvers e.g. CPLEX 6.5, OSLMSLV 3.0.

\subsection{Node Heuristics}

This feature sets the value of integer variables at selected nodes in the $B \& B$ tree and checks whether it can find an integer feasible solution from it. The Node Heuristics are specific to the solver. The CPLEX 6.5 and OSLMSLV 3.0 MILP solvers use heuristics to quickly find an integer feasible solution at the B\&B node. A detailed discussion of this feature is outside the scope of this thesis. 


\section{Branching Variable Selection Methods: State of the Art}

In the branching variable selection methods presented by Linderoth and Savelsbergh [1999], Dakin [1965], Benichou et al. [1971], Gauthier and Ribiere [1977] and Eckstein [1994], the primary objective is to select a non-integral valued integer variable as the branching variable in order to maximize the degradation in the objective function value at the optimal solution of the child node LP relaxation. The primary goal of this approach is to get a tighter upper bound value on the unsolved nodes. The advantages of this approach are described in Section 2.1.5. As pointed out by Linderoth and Savelsbergh [1999], most of the branching variable selection methods either estimate degradation in the objective function value of the LP relaxation or provide lower bounds on the degradation. The estimation methods are based on pseudo-costs introduced by Benichou et al. [1971]. Before continuing with the discussion on different branching schemes, pseudo-cost is explained here as it is used by several methods.

A pseudo-cost (PC) estimates the change in the objective function value of an LP for a unit change in the value of an integer variable. The pseudo-cost is calculated separately for the upper $(P C U)$ and the lower $(P C L)$ branch child nodes. Different integer variables have different pseudo-cost values. Let's say, at node $\mathrm{k}$ in the $\mathrm{B} \& \mathrm{~B}$ tree, the objective function value at the optimal solution of the LP relaxation problem is $F_{k}$. Two child nodes $(n+1$ and $n+2)$ are created by branching on an integer variable $\mathrm{x}_{\mathrm{i}}$ that has noninteger value $\left(v_{i}\right)$ in the optimal solution of the LP relaxation problem. The optimal objective function values of the LP relaxation problems at nodes $n+1$ and $n+2$ are $F_{n+1}$ and $F_{n+2}$ respectively. The Pseudo-costs for variable $x_{i}$ are calculated as follows: 


$$
\begin{aligned}
& P C L_{i}=\frac{\left|F_{n+1}-F_{k}\right|}{\left|v_{i}-\left\lfloor v_{i}\right\rfloor\right|} \\
& P C U_{i}=\frac{\left|F_{n+2}-F_{k}\right|}{\mid v_{i}-\left[v_{i}||\right.}
\end{aligned}
$$

Now suppose variable $\mathrm{x}_{\mathrm{i}}$ has non-integer value $\left(\mathrm{w}_{\mathrm{i}}\right)$ in the LP relaxation optimal solution of any other node, say node p.

Let $E L_{i}=$ Estimated degradation in the objective function value of the LP relaxation optimal solution at the lower branch child node of node $\mathrm{p}$ that is created by branching on an integer variable $\mathrm{x}_{\mathrm{i}}$.

$E U_{i}=$ Estimated degradation in the objective function value of the LP relaxation optimal solution at the upper branch child node of node $\mathrm{p}$ that is created by branching on an integer variable $\mathrm{x}_{\mathrm{i}}$.

The degradation in the objective function value at the unsolved child nodes of node $\mathrm{p}$ can be estimated using the LP relaxation optimal solution of node $\mathrm{p}$ and the previously calculated pseudo-costs values as follows:

$$
\begin{aligned}
& E L_{i}=P C L_{i} \times\left(\mid w_{i}-\left\lfloor w_{i}||\right)\right. \\
& E U_{i}=P C U_{i} \times\left(\left|w_{i}-\left\lceil w_{i}\right\rceil\right|\right)
\end{aligned}
$$

To compute the values of pseudo-costs of the variables at any $\mathrm{B} \& \mathrm{~B}$ node, one has to solve both child node LP problems. Since there can be many fractional valued integer variables in the optimal solution of the LP relaxation problem, it is computationally expensive to calculate the exact value of the pseudo-costs for each integer variable at 
each node in the $\mathrm{B} \& \mathrm{~B}$ tree. Most of the existing branching variable selection methods that are based on pseudo-cost try to estimate the actual pseudo-cost value by solving fewer extra LP relaxation problems. An important observation by Benichou et al. [1971] and later reconfirmed by Linderoth and Savelsbergh [1999] is that the pseudo-cost values of the integer variables in a particular branch direction remain constant throughout the $B \& B$ tree with the exception of only a few nodes. This means that once the pseudo-cost of a variable is computed then it can be used to make the branching decision throughout the $\mathrm{B} \& \mathrm{~B}$ tree without having to re-compute it at other nodes.

This section will present some known branching variable selection methods for $\mathrm{B} \& \mathrm{~B}$ based on LP relaxation. These methods are introduced and discussed in various research papers and in some papers the experimental results are provided to show their effectiveness. These methods can be categorized into two classes:

\section{- Static branching methods}

In such methods, integer variables are kept in a list in decreasing order of their branching priority. To select a branching variable at node $\mathrm{k}$, starting from the top of the list, the first integer variable having non-integral value in the LP relaxation optimal solution at node $\mathrm{k}$ is selected. An ordered list of integer variables may be available before the $B \& B$ algorithm is started or can be developed as the $B \& B$ method continues. In the latter case, the relative order of the variables in the list does not change.

\section{- Dynamic branching methods}

In such methods, the branching variable at a particular node is selected by evaluating the integer variables with non-integral value in the LP relaxation optimal solution. 
Evaluation of variables is based on certain rules that are specific to each method. These methods do not maintain a fixed priority list of integer variables but perform some computation at each node of the $B \& B$ tree to select the branching variable. Such computations can be very simple, for example comparing the fractional part of the value of the integer variables in the LP relaxation optimal solution, or can be very complex, for example solving certain LPs, or somewhere in between. Dynamic branching methods are generally more expensive, in terms of time and memory, than static branching methods. However the quality of the selected branching variable is usually much better in the dynamic methods, which decreases the overall time to solve the MILP problems. There is a tradeoff between the extra effort spent in selecting an integer variable among the candidate branching variables and the overall gain in the effort to reach the optimal solution of the MILP problem. Sometimes this gain is not worth the extra effort spent. This tradeoff should be carefully measured and is usually done by performing experiments (as done by Linderoth and Savelsbergh [1999], Benichou et al. [1971] and Gauthier and Ribiere [1977]) on the selected MILP problems.

\subsection{Static branching methods}

The following sections discuss some of the static branching methods.

\subsubsection{Using prior knowledge of the problem}

This scheme was briefly described by Benichou et al. [1971]. In this method, prior knowledge of the problem being solved is used to determine the priority of the integer 
variables for branching. Consider the following example. A company wants to construct a few buildings in a city. There are $n$ construction sites on which $m$ buildings $(m<n)$ should be constructed. On each site, at most one building can be constructed. Each employee in this company is assigned to exactly one building. One way to model this problem is to associate a binary variable $x_{i}$ with each site whose value will indicate whether a building is constructed at that site $\left(x_{i}=1\right)$ or not $\left(x_{i}=0\right)$. The value of another binary variable $y_{\mathrm{ij}}$ will indicate if worker $\mathrm{i}$ is assigned to a building at site $\mathrm{j}$. The decision to construct a building at site $\mathrm{i}$ is more important and should be made before the decision to assign the workers to that building. So one would branch on $\mathrm{x}_{\mathrm{i}}$ variables before $\mathrm{y}_{\mathrm{ij}}$ variables. The ordering within $\mathrm{x}_{\mathrm{i}}$ variables or within $\mathrm{y}_{\mathrm{ij}}$ variables can be done randomly or based on some other knowledge of the problem. The branching priority is either entered by the user or automatically derived by the modeling software before solving the MILP problem.

\subsubsection{Coefficient of variables in objective function}

In this method, the integer variables are added to a list in decreasing order of absolute value of their coefficient in the objective function. As pointed out by Linderoth and Savelsbergh [1999], if the variables are unrelated in the MILP problem then the objective function coefficient of a variable indicates the amount of change in the objective function value for a unit change in the value of that variable. This method is very simple to implement and it does not require any prior knowledge of the problem. Benichou et al. [1971] and Gauthier and Ribiere [1977] used this method with some minor variations for experimental comparison with other methods. Some results are shown in Section 3.1.3. 


\subsubsection{Static ordering based on pseudo-cost}

Benichou et al. [1971] has suggested explicitly computing the pseudo-cost of each variable and building a list of integer variables in decreasing order of max(lower branch pseudo-cost, upper branch pseudo-cost). The branching variable selection scheme is to select the variable highest in this list that has fractional value in the LP relaxation optimal solution. To build the list, the pseudo-costs of the variables are calculated in the previous run of the same problem. The experimental results show that the number of simplex iterations performed to reach the optimal solution of the MILP problem and to prove optimality are significantly smaller compared to the branching scheme that uses the absolute value of the objective function coefficients.

Gauthier and Ribiere [1977] provided a branching variable selection method called "Automatic Ordered branching" in which the integer variables are sorted in decreasing order based on the estimation of degradation in the objective function value. These estimates are calculated using the pseudo-costs of the variables. Pseudo-cost of the variables is calculated as needed and once it is calculated it is reused at other nodes. The list is not available in the beginning but it is built as the search continues. The branching variable is selected from the top of the list. At any B\&B node, if the list is empty or if all variables in the list have integer values then the integer variable that has the maximum sum of the estimated degradation on both branches is selected as the branching variable and is also added at the end of the list. Since explicitly computing the pseudo-cost requires solving dummy LP problems, which is computationally expensive, a limit is imposed on the number of simplex iterations for calculating the pseudo-cost. The 
experimental results given by Gauthier and Ribiere [1977] indicate that the B\&B strategy using automatically ordered branching is effective in finding a first integer feasible solution of better quality but it takes more time. Also this method is only effective for problems having fewer integer variables compared to the total number of variables. Please note that other decisions in the B\&B methods that might affect the results are not provided here but can be found in Gauthier and Ribiere [1977].

\subsection{Dynamic branching methods}

The following sections discuss some of the dynamic branching methods.

\subsubsection{Closest to integer feasibility}

A simple way of selecting the branching variable dynamically is to judge its closeness to integer feasibility based on the fractional part of its value in the LP relaxation optimal solution. A variable with the optimal value closer to the nearest integer value is considered closer to integer feasibility. One can then select the fractional valued integer variable that is closest to integer feasibility as the branching variable. Dakin [1965] used a similar branching variable selection scheme and found that the results were not as good (in terms of simplex iterations) as the ones obtained by using the single dual simplex pivot scheme (see Section 3.2.3).

\subsubsection{Dynamic branching based on pseudo-cost}

Gauthier and Ribiere [1977] presented this method that explicitly computes the pseudocost of the fractional valued integer variables. Dummy LP relaxation problems are created 
by separately branching on each fractional valued integer variable in the LP relaxation optimal solution. This computation is only done once for each integer variable when they have a fractional value in the LP relaxation optimal solution. The computed pseudo-cost values are then reused to make branching decisions at other nodes. The experiments show that explicitly computing the pseudo-cost is time consuming and so a limit is imposed on the number of simplex iterations allowed for solving dummy LP relaxation problems. If the computation is terminated prematurely then the pseudo-cost is calculated based on the partial solution. The pseudo-cost values are replaced by the actual observed value once the child nodes are solved after branching. The pseudo-costs values are used to estimate the degradation in the objective function value that will be obtained by branching on a candidate variable. The candidate variable that gives largest sum of estimated degradation in the objective function value of both branches is selected as the branching variable.

Gauthier and Ribiere [1977] developed a branching scheme that uses Automatic Ordered branching (Section 3.1.3) until the first integer feasible solution is found and then uses the dynamic branching scheme based on pseudo-cost value of the variables. Such a scheme was able to quickly find the optimal solution (based on the number of simplex iterations) of MILP problems having relatively fewer integer variables. The comparison was done against the $B \& B$ strategy using a branching scheme that is a minor variation of the static ordering scheme based on the objective function coefficients. Please note that other decisions in the B\&B methods might affect the results and are not provided here but can be found in Gauthier and Ribiere [1977].

Eckstein [1994] used a branching variable selection method that keeps track of the pseudo-cost of the integer variables on up and down branches. The average of these 
values is used for initializing the pseudo-cost of an integer variable that has never been branched on. Once the integer variable is selected as the branching variable, its pseudocost is set to the observed value. During each subsequent selection of the same variable as the branching variable, the pseudo-cost is updated to the average of the observed pseudocost values. This scheme does not require the solution of dummy LP problems. Forest et. al. [1974] suggested that one way to update the pseudo-cost of the integer variables is to use the last observed value.

Linderoth and Savelsbergh [1999] suggest that during the B\&B process, if an integer variable, which has never been branched on before, has a fractional value in the optimal solution of the LP relaxation problem then its pseudo-cost should be explicitly computed. As done by Gauthier and Ribiere [1977], Linderoth and Savelsbergh [1999] also suggest setting a limit on the number of simplex iterations performed for explicitly computing the pseudo-cost. However the limit should be set to a higher value in order to get closer to the "true" pseudo-cost value. After the initialization, the pseudo-cost of an integer variable is updated by averaging its observed pseudo-cost values. The objective function degradation is computed based on the pseudo-cost and the branching variable is selected as the one with the maximum sum of degradation on up and down branches. By performing experiments on various pseudo-cost initialization and update methods, Linderoth and Savelsbergh [1999] showed that in most of the cases the above method is the best branching variable selection scheme for finding the optimal solution of MILP problems. 


\subsubsection{Single dual simplex pivot}

Dakin [1965] introduced a method that selects the fractional valued integer variable that gives the largest degradation in the objective function value of the LP relaxation problem during the first dual simplex pivot. The dual simplex pivot is performed after changing the variable bound. The degradation in the objective function value of the LP problem during the first dual simplex pivot represents a lower bound on the degradation of the objective function value that can be attained at the optimal solution of the LP problem. This scheme tries to maximally degrade the objective function value at the optimal solution of the child nodes, increasing the chances of pruning the child nodes.

Beale [1968] uses a similar technique for selecting the branching variable from the fractional valued basic integer variables. Tomlin [1969] extended this method to also consider the non-basic integer variables. It uses the simple fact that the non-basic integer variables, if required to take a non-zero value, must have a value of 1 at least. Also the change in the objective function value is equal to the reduced cost of the variable. The reduced cost value along with the known finite integer feasible solution is used to decide if the non-basic integer variable can have non-zero value in the current branch and still find a better integer feasible solution. The experiment performed by Tomlin [1969] shows that this method, compared to the original method introduced by Beale [1968] and Small [1965], resulted in a considerable improvement in the number of simplex iterations and the number of branches that are required to reach the optimal solution of the pure or nearpure integer problems. 


\subsubsection{Strong branching}

The Strong branching scheme [Bixby 1996 ] performs a number of dual simplex pivots in order to get the lower bound on the degradation in the objective function value at the LP relaxation optimal solution of the child node closer to the "true" degradation value. At each node in the $B \& B$ tree, some of the integer variables having non-integer values in the LP relaxation optimal solution are selected. The selected number of dual simplex pivots is carried out after branching on each of these integer variables individually in order to select the branching variable that gives a larger degradation in the objective function value of the LP relaxation problem.

Linderoth and Savelsbergh [1999] conducted experiments to compare different branching schemes based on dual simplex pivot and observed that the single dual simplex pivot branching scheme performs better than the strong branching scheme. The strong branching scheme used in this comparison selects a sub-set of the integer variables based on the fractional part of their value in the LP relaxation optimal solution. The integer variables having the fractional part of their value in the LP relaxation optimal solution closer to 0.5 are selected.

\subsubsection{Combination of objective function coefficient and fractional part of integer variable}

Padberg and Rinaldi [1991] used this scheme in their branch-and-cut algorithm for solving large-scale traveling salesman problems. After solving the LP relaxation problem at each node of the $B \& B$ tree, a subset $l$ of the fractional valued integer variables is constructed based on the closeness of the fractional part of their value in the LP relaxation 
optimal solution to 0.5 . The integer variables in the subset $I$ with the largest absolute value of the objective function coefficient are selected to construct another subset $I_{2}$. The branching variable is then selected from $I_{2}$ such that the absolute value of its objective function coefficient is large and the fractional part of its value in the LP relaxation optimal solution is close to 0.5 .

\subsubsection{Combination of estimation and lower bound methods}

Linderoth and Savelsbergh [1999] suggest combining the pseudo-costs and the lower bound methods for selecting the branching variable. The estimate of the degradation in the objective function value after branching on the variable $x_{j}$ is calculated as:

$$
E_{j}=\beta_{0}+\beta_{1} D_{j}+\beta_{1} L_{j}
$$

Where $\beta_{0}, \beta_{1}, \beta_{2}$ are constant and their values are determined using regression testing. $D_{j}$ is the estimated degradation in the objective function value which is calculated using the pseudo-cost method (see Section 3.2.2) and $\mathrm{Lj}$ is the lower bound on the degradation in the objective function value as computed by performing the dual simplex pivot. The experimental results suggest that this method has better average performance than using the pseudo-cost or the lower bounds method alone.

\subsection{Special Ordered Sets branching (SOS branching)}

If the MILP problem contains a constraint of the form $\Sigma_{j \in I 1} x_{j}=1$ for some set 11 of the integer variables then a special type of branching called SOS1 branching can be used. All $\mathrm{x}_{\mathrm{j}}$ variables in the above constraint are binary variables. At any point in the $\mathrm{B} \& \mathrm{~B}$ tree, if 
the $\mathrm{LP}$ relaxation optimal solution contains one or more $x_{j}$ variables with fractional values then the SOS1 branching scheme selects a subset $\mathrm{T} \subseteq \mathbb{I}$ and adds a constraint $\Sigma_{\mathrm{j} \in \mathrm{T}}$ $\mathrm{x}_{\mathrm{j}}=0$ in one branch and a constraint $\Sigma_{\mathrm{j} \in 11 \mathrm{~T}} \mathrm{x}_{\mathrm{j}}=0$ in the another branch. As pointed out by Linderoth and Savelsbergh [1999], branching on a single binary variable that is not "important" can lead to an LP relaxation optimal solution that is almost the same as the parent node optimal solution. In this case, we have made very little progress toward reaching the final solution. If the MILP problem contains a constraint of the form $\Sigma_{j \in I 1} x_{j}$ $=1$ then simultaneously setting several binary variables to 0 can lead to larger changes in the LP relaxation solution of the child node. To determine the set $T$ of binary variables, a special constraint known as the reference row is used. The reference row provides an ordering of the variables based on certain criterion (generally in increasing order of the variable's coefficient in the constraint). The value of the variables in the LP relaxation optimal solution is substituted in the reference row to determine a branching point. The set $\mathrm{T}$ consists of all variables in the reference row that are left of the branching point (and also including the branching point). The following example will clarify the point:

Suppose a building has to be constructed at one of four possible locations. The values of the binary variables $x 1, x 2, x 3$ and $x 4$ will indicate the location at which the building will be constructed. So the following constraint is added to the problem:

$$
x_{1}+x_{2}+x_{3}+x_{4}=1
$$

The objective function is to minimize the cost of the construction. The following is the size constraint for constructing this building (reference row):

$$
5 x_{1}+10 x_{2}+20 x_{3}+30 x_{4} \leq 40
$$


In the LP relaxation optimal solution at any $B \& B$ node, if the value of the variables are $x_{1}$ $=0.30, \mathrm{x}_{2}=0.65, \mathrm{x}_{3}=0.43, \mathrm{x}_{4}=0.35$ then substituting these values in the above constraint will give a LHS value of $5(0.30)+10(0.65)+20(0.43)+30(0.35)=27.1$. The LP relaxation optimal solution can be interpreted as suggesting to either construct a building of size less than or equal to 20 or greater than or equal to 30 in order to minimize the construction cost. Since the coefficient of third variable (i.e. $x 3$ ) in the reference row is 20 , index 3 is selected as the branching point and the set $T=\{x 1, x 2, x 3\}$.

Special Ordered Sets of type 2 (SOS2) require that at most two variables in the reference row have non-zero values in the final solution. Also these non-zero variables must be adjacent in the reference row. SOS2 branching can be used for finding the optimal solution of an application containing a piecewise linear approximation of a nonlinear function of a single argument. Special Ordered Sets were introduced by Beale and Tomlin [1970] and are also discussed in Beale [1979] and Linderoth and Savelsbergh [1999]. 


\section{Node Selection Methods: State of the Art}

Node selection is another important decision that affects the performance of $\mathrm{B} \& \mathrm{~B}$ based on the LP relaxation. Several node selection schemes select nodes that have a higher potential of giving a good integer feasible solution. Some of the commonly used node selection methods are described below.

\subsection{Depth First Search (DFS)}

Dakin [1965] first used this node selection method for solving MILP problems. After creating the child node(s) during branching, this method always selects one of the newly created nodes as the next node to solve. In this way, the search is continued deeper into the $B \& B$ tree until an integer feasible solution is found or the node becomes infeasible. In the latter case, the last created unsolved node is selected as the next node to solve. There are two main advantages of this method:

- As pointed out by Dakin [1965], LP problems at the parent and its child nodes are very similar and so the parent node LP optimal solution can be used as a starting point for solving the LP at the child nodes. Keeping track of the LP optimal solution at each node during any $\mathrm{B} \& \mathrm{~B}$ process is expensive in terms of memory. DFS method partly overcomes this problem as one of the child nodes is solved next, so its parent node optimal solution is used immediately as a hot start.

- Since the DFS method prefers to search deeper into the B\&B tree, there are higher chances of finding an integer feasible solution faster. 
The main disadvantage of this method is that it explores "unnecessary" nodes at the bottom of the $\mathrm{B} \& \mathrm{~B}$ tree that might have been removed during pruning if a good integer feasible solution was known earlier. Thus the DFS method can increase the overall time to reach an optimal solution of the MILP problem.

\subsection{Best First Search (BFS)}

BFS selects a node, among all the candidate nodes, with the best objective function value of the LP relaxation optimal solution at the parent node. By following the best branch, it increases the chances of finding a good integer feasible solution. It also prevents solving "unnecessary" nodes that might be targets for pruning once an integer feasible solution (or a better integer feasible solution than the current one) is found. The main disadvantage of this method is that it favors nodes that are higher in the $B \& B$ tree which may cause delay in finding an integer feasible solution and pruning the nodes from the $B \& B$ tree. Land and Doig [1960], the inventors of B\&B based on the LP relaxation, used this node selection strategy.

\subsection{Estimation based on pseudo-cost}

Benichou et al. [1971] estimated the best integer feasible solution that can be obtained from the descendant of a given node using the pseudo-costs of the integer variables at that node. Pseudo-cost calculation is described in Section 3. For a minimization problem, an estimate of the best integer feasible solution that can be obtained from the descendant of a node, say $\mathrm{k}$, is calculated as follows: 


$$
E_{k}=F_{k}+\sum_{j=1}^{q} \min \left(P C L_{j} \times f_{j k}, P C U_{j} \times\left(1-f_{j k}\right)\right)
$$

where,

$F_{k}=$ Objective function value of the LP relaxation optimal solution at node $\mathrm{k}$

$\mathrm{PCL}_{\mathrm{j}}=$ Pseudo-cost of integer variable $\mathrm{j}$ associated with the lower branch

$\mathrm{PCU}_{\mathrm{j}}=$ Pseudo-cost of integer variable $\mathrm{j}$ associated with the upper branch

$f_{j k}=$ Fractional part of the value of integer variable $j$ in the LP relaxation optimal solution at node $\mathrm{k}$.

$q=$ Number of integer variables in the MILP problem.

The node selection strategy here is to perform the depth first search, selecting the node with the best estimate among the newly created child nodes. If an integer feasible solution is found or the node becomes infeasible then the node with the best estimate (similar to BFS strategy) is selected as the next node to solve. The experimental results from Benichou et al. [1971] show that this strategy performs better, in terms of the number of $\mathrm{B} \& \mathrm{~B}$ nodes and the number of simplex iterations, than DFS strategy for finding the optimal solution of most MLP problems.

\subsection{Best Projection}

This method keeps track of the sum of integer infeasibilities in the LP relaxation optimal solution of each solved node. For node $\mathrm{k}$, the sum of integer infeasibilities $\left(\mathrm{I}_{\mathrm{k}}\right)$ is calculated using the following formula: 


$$
I_{k}=\sum_{j=1}^{q} \min \left(f_{j k},\left(1-f_{j k}\right)\right)
$$

where,

$f_{j k}=$ Fractional part of the value of variable $j$ in the LP relaxation optimal solution at node k.

$q=$ Number of integer variables in the MLP problem.

For a minimization problem, an estimate $\left(\mathrm{E}_{\mathrm{k}}\right)$ of the objective function value of an integer feasible solution that can be obtained from the descendant of node $\mathrm{k}$ is calculated as follows:

$$
E_{k}=\frac{F_{k}}{\alpha}+\left(\frac{Z_{b e s t}-F_{0}}{I_{0}}\right) \times I_{k}
$$

where,

$F_{k}=$ Objective function value of the LP relaxation optimal solution at node $k$.

$Z_{\text {best }}=$ Objective function value of the best integer feasible solution that is known.

$\mathrm{F}_{0}=$ Objective function value of the LP relaxation optimal solution at the root node.

$I_{0}=$ Sum of integer infeasibilities at the root node.

$\alpha=$ A constant whose value can be set by the user. $0<\alpha \leq 1$

The term $\left(Z_{\text {best }}-F_{0}\right)$ in the above equation represents the change in the objective function value of the LP relaxation optimal solution by removing $\mathrm{I}_{0}$ integer infeasibilities as we proceed from the root node to the node having the best known integer feasible solution. This method selects the node with the best estimation of the objective function value of an integer feasible solution that can be obtained from its descendants. The user 
can set the value of the parameter $\alpha$. Values closer to 0 will force the node selection to be mainly driven by the objective function value of the LP relaxation optimal solution. Values closer to 1 will force the node selection to be mainly driven by the integer infeasibilities of the nodes. Hirst [1969] and Mitra [1973] were among the first to describe this method.

\subsection{Backtracking}

The DFS strategy is effective in finding the integer feasible solution faster but there are chances of exploring "unnecessary" nodes. The BFS strategy works towards finding the global optimal solution faster but it can cause delay in finding the first integer feasible solution. As explained in Section 2, finding the first integer feasible solution faster will cause earlier pruning of the $\mathrm{B} \& \mathrm{~B}$ tree and will reduce the search space to explore. $\mathrm{A}$ node selection strategy is developed that uses the DFS strategy until the first integer feasible solution is found and then uses the BFS (or estimation or projection) strategy. This clearly separates the B\&B procedure into two parts - the DFS part and the BFS part. However one can also mix these two by using backtracking. The backtracking method performs the DFS until either an integer feasible solution is found or the node becomes infeasible or the backtracking condition is violated. In these cases, the DFS is terminated and the next node is selected using a different node selection strategy (e.g. BFS). This is called backtracking. Again the algorithm continues using the DFS and so on.

The backtracking condition at node $\mathrm{k}$ can be (but is not limited to) one of the following: 
- For a minimization problem, the backtracking condition used by the CPLEX 6.5 MILP solver [ILOG Corporation 1999b] is as follows:

$$
\text { If }\left(\left(F_{k}-F_{\text {best }}\right)<\left(F_{\text {incumbent }}-F_{\text {best }}\right) \times \text { BacktrackTolerance }\right) \text { then do not backtrack }
$$

where,

$F_{k}=$ objective function value of LP relaxation solution at node $\mathrm{k}$.

$F_{\text {best }}=$ Best objective function value among candidate nodes

$F_{\text {incumbent }}=$ objective function value of known best integer feasible solution .

BacktrackTolerance $=$ any number between 0 and 1.

The value of the BacktrackTolerance parameter can be set by the user. A high BacktrackTolerance value causes the backtrack condition to be violated less frequently resulting in a search that is closer to the DFS strategy. A low value will cause a search that is closer to the BFS (or best estimate or other) strategy.

- Gauthier and Ribiere [1977] proposed a backtracking condition based on the estimation method. For a minimization problem, the backtracking condition used at node $\mathrm{k}$ is:

$$
\text { If }\left(E_{k} \leq H_{n}+\rho \times\left(H_{n}-F_{0}\right)\right) \text { then do not backtrack }
$$

where,

$E_{k}=$ Estimation of the objective function value of the best integer feasible solution that can be obtained from the descendants of node $\mathrm{k}$. See Section 4.3 .

$\mathrm{H}_{\mathrm{n}}=\min ($ Estimate of all candidate nodes using the calculation shown in Section 4.3) $F_{0}=$ Objective function value of the LP relaxation optimal solution at the root node.

$\rho$ is a constant. Generally set to 0.5 


\section{Research Question}

As discussed in Section 3 most of the existing branching variable selection schemes use objective function measures such as pseudo-costs to select the branching variable. In contrast, it is the active constraints that influence the optimal solution of the LP relaxation problem (Section 2.2). In the B\&B process, considering the active constraints of the LP relaxation optimal solution while making the branching decision at a node can be effective in "moving" the optimum point of the LP relaxation problem at the child nodes. In this approach, selection of the branching variable is based on the constraints rather than on objective function measures and so it is expected to have more impact on feasibility than on the objective function value. Section 2.1.5 explained the importance of finding a good initial feasible solution of the MILP problem faster and its impact on the search for the optimal solution.

Research question: Can measures based on the active constraints in the LP relaxation optimum solution at a branch and bound node be used to select the branching variable such that integer feasibility is achieved more quickly?

Node selection is another important decision in the B\&B process. Different node selection schemes should be considered as part of the overall algorithm. The goal is to select the scheme that, along with the proposed branching variable selection method, finds the first integer feasible solution of the MILP problem faster. As explained in Sections 4.1 and 4.5, DFS node selection is effective in finding an integer feasible 
solution quickly and is generally used in the $B \& B$ process until the first integer feasible solution is found. The integer infeasibilities (i.e. violation of integrality constraints by the variables) in the LP relaxation optimal solution at the node and the constraints associated with such variables can be used to estimate the closeness of that node to integer feasibility.

Secondary question 1: Can node selection schemes that choose a node based on its estimated closeness to integer feasibility, along with a branching variable selection scheme based on the active constraints, be used to achieve integer feasibility more quickly than the DFS strategy?

Finding a good integer feasible solution of the MLP problem faster causes earlier pruning of the B\&B tree and can lead to the optimal solution faster.

Secondary question 2: What is the impact of the branching variable selection scheme based on the active constraints on the speed-to-optimality in MILP solutions?

After solving the LP problem at a node, the dual cost of the constraint is generally used to determine if the constraint is active. If the dual cost is non-zero then the constraint is active. But degeneracy in the LP problem can cause the LP solver to set the dual cost of some of the constraints to zero even when they are tight (i.e. the difference between LHS and RHS of the constraint is less than the feasibility tolerance of the solver) at the optimum point. Since we are interested in all constraints that influence the LP relaxation 
optimum point, we cannot directly use the dual cost value of the constraints to determine if the constraint is active. In this thesis, we define the active constraints to be the equality constraints and those in which the absolute value of the difference between LHS and the RHS is less than the feasibility tolerance of the MILP solver. 


\section{Dynamic Variable Ordering Schemes Based on Active Constraints}

Let us consider the effect on the optimum point of an LP problem of changing the bounds on a variable having a non-zero coefficient in an active constraint. Consider the following example:

Objective: $\operatorname{Max} z=2 x+y$

Constraint $\mathrm{A}: 3 \mathrm{x}+\mathrm{y} \leq 7.5$

Constraint B: $1.43 \mathrm{x}+\mathrm{y} \leq 5$

$\mathrm{x}, \mathrm{y} \geq 0$

$\mathrm{x}, \mathrm{y}$ are integer variables

The graphical solution of the LP relaxation of the above problem is shown in Figure 9 (a).

Constraints $\mathrm{A}$ and $\mathrm{B}$ are the active constraints and the optimal solution of the LP relaxation is at the point $(1.59,2.72)$.

Consider changing the upper bound of variable $y$, which has a non-zero coefficient in active constraints $\mathrm{A}$ and $\mathrm{B}$, to the nearest integer value less than its current optimal value (i.e. 2). The graphical solution of this modified LP problem is shown in the lower region of Figure 9 (b). The new upper bound constraint causes change in the feasible region and moves the optimal point to the intersection of constraint $A$ and the new upper bound constraint on variable $y$, i.e. to the point $(1.83,2.0)$. Now consider changing the lower bound of variable $y$ in the original problem to the nearest integer value greater than its current optimal value (i.e. 3). The graphical solution of this modified LP problem is shown in the upper region of Figure 9 (b). The new lower bound causes change in the 
feasible region and moves the optimal point to the intersection of constraint $B$ and the new lower bound constraint on variable $y$, i.e. to the point $(1.39,3.0)$.

If we change the upper bound on variable $\mathrm{x}$, which also has a non-zero coefficient in active constraints $\mathrm{A}$ and $\mathrm{B}$, to the nearest integer value less than its current optimal value (i.e. 1) then the optimal point will move to $(1.0,3.57)$ (shown in the left region of Figure 9 (c)). Similarly if we change the lower bound on variable $\mathrm{x}$ in the original LP problem to the nearest integer value greater than its current optimal value (i.e. 2 ) then the optimal point will move to $(2.0,1.5)$ (shown in the right region of Figure 9 (c)). Hence, as shown in Figure 9, branching on variable $\mathrm{x}$ has a larger impact on moving the optimal point of the LP relaxation problem compared to branching on variable $y$. Thus changing the bounds of different variables appearing in the active constraints has different impacts on moving the LP optimum point.

As explained in Section 2.1.5, in the Branch routine of the B\&B algorithm, the bounds on the branching variable are changed and child nodes are created. The dynamic branching variable selection schemes based on active constraints try to select the fractional valued integer variable that causes the most change in the active constraints. Changes in the active constraints will move the optimal point of the LP relaxation at the child nodes. The intent of the proposed dynamic branching schemes is to move the optimal point of the LP relaxation at the child nodes as much as possible. This will likely move the LP relaxation optimum point at one of the child nodes closer to the integer feasible solution. 


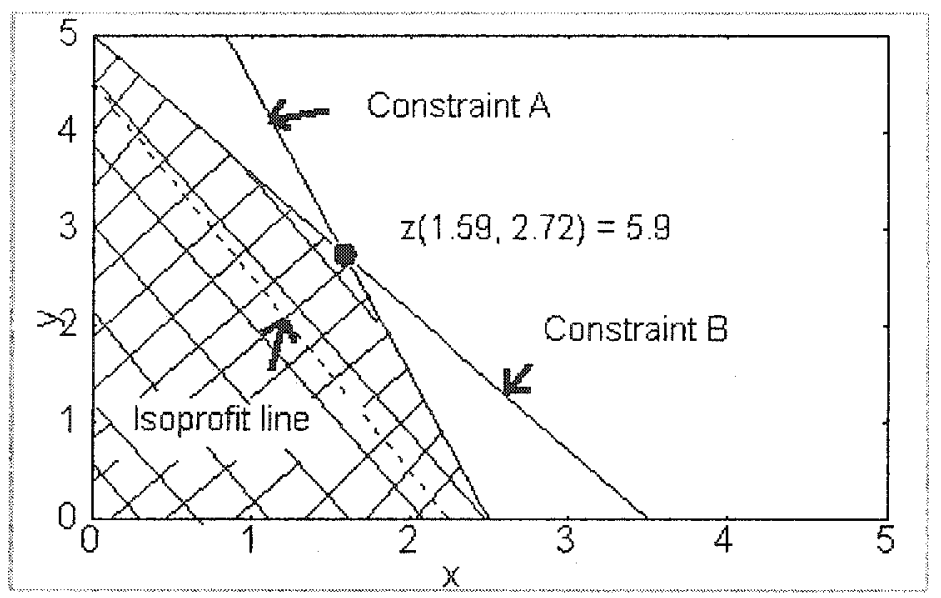

(a) LP relaxation optimal solution

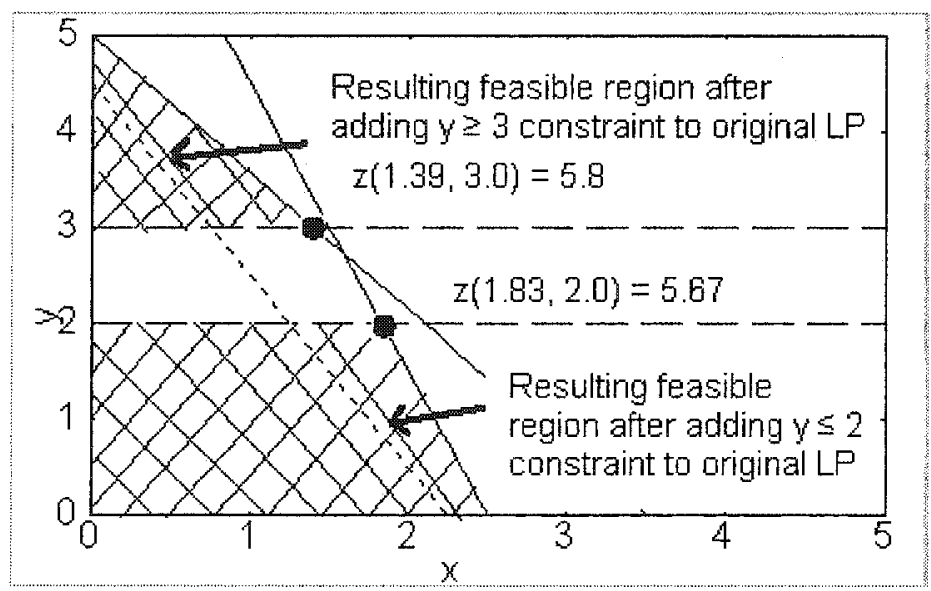

(b) LP relaxation optimal solutions after branching on variable $y$

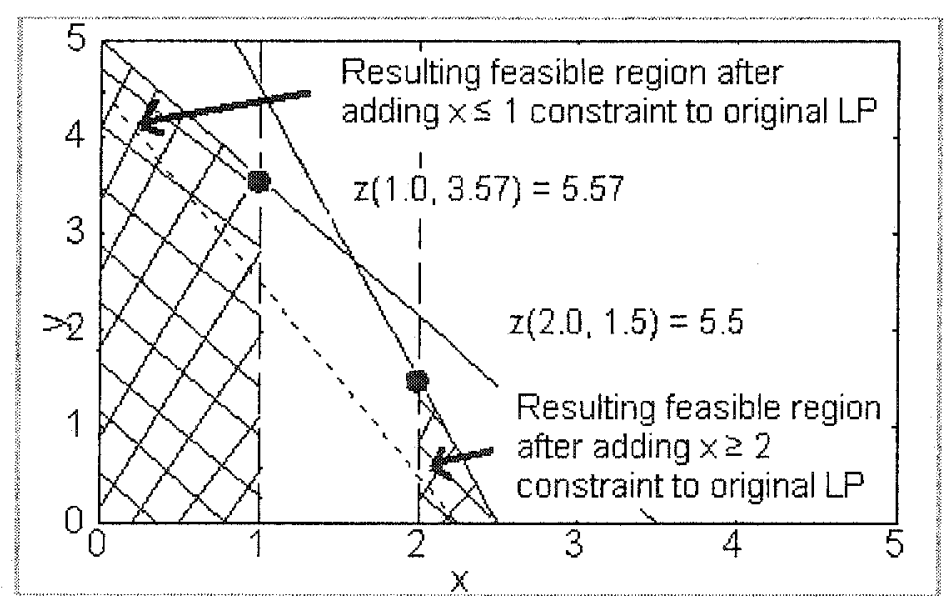

(c) LP relaxation optimal solutions after branching on variable $\mathrm{x}$

Figure 9: Effect on the optimal point of the LP of changing the bounds on a variable 
The dynamic branching schemes based on the active constraints in the LP relaxation optimal solution will be referred to as Active Constraint Schemes. In most Active Constraint Schemes, the weight of the fractional valued integer variable is calculated separately for each active constraint based on its potential for bringing change to that constraint. The branching variable selected is the one with the maximum sum of the weights over all active constraints. Different parameters (e.g. the number of active constraints in which the coefficient of the fractional valued integer variable is non-zero, the coefficient of the fractional valued integer variable in an active constraint, etc.) are used to compute the weight of the fractional valued integer variables in the active constraints. The rationale for each proposed scheme is described below. The following example is used to explain the selection of the branching variable in the Active Constraint Scheme described below.

$$
\begin{aligned}
& \text { Max } z=3 v-4 w+x-2 y \\
& \text { Constraint } P: 8 v+x-y \leq 9 \\
& \text { Constraint } Q:-w+2 x+y \leq 5 \\
& \text { Constraint } R: 3 v+4 w+2 x \leq 10 \\
& v, w, x, y \geq 0 \\
& v, x \text { and } y \text { are integer variables }
\end{aligned}
$$

The LP relaxation optimal solution at the root node of the $B \& B$ tree is $z(v, w, x, y)=$ $\mathrm{z}(0.8125,0,2.5,0)=4.9375$. So the candidate branching variables are $\mathrm{v}$ and $\mathrm{x}$. Constraints $\mathrm{P}$ and $\mathrm{Q}$ are the active constraints at the LP relaxation optimal solution and their dual costs are 0.375 and 0.3125 respectively. 


\subsection{Active Constraint Schemes based on the number of active constraints containing the fractional valued integer variable}

How much impact a candidate variable has on an active constraint depends on its occurrence in that constraint. This observation underlies the methods (schemes A to D) described below. The weights of the candidate variables in an active constraint calculated by schemes $B, C$ and $D$ can be normalized by multiplying them with the RHS of the constraint. This will eliminate the scaling effects. However the un-normalized results that are obtained using scheme D are good (see Table 1) and schemes E, F and G (described later) are normalizing the weights in a different way. Hence schemes B, C and D are not normalizing the weight of the candidate variables in an active constraint.

\section{- Active Constraint Scheme A:}

Rationale: A simple count of the number of active constraints in which a candidate variable occurs should give a measure of its impact on the active constraints.

For active constraint $(\mathrm{A} 1)$, the weight $\left(\mathrm{WN}_{\mathrm{X} 1 \mathrm{Al}}\right)$ of fractional valued integer variable (X1) having a non-zero coefficient in that constraint is 1 otherwise the weight is 0 . Thus the total weight of each fractional valued integer variable is the number of active constraints in which it has a non-zero coefficient.

In the given example, the weight of each fractional valued integer variable in constraints $\mathrm{P}$ and $\mathrm{Q}$ is calculated as follows: 


\begin{tabular}{|l|c|c|}
\hline & $\mathbf{W N}_{\mathbf{v}}$ & $\mathbf{W N}_{\mathbf{x}}$ \\
\hline Constraint $\mathbf{P}$ & 1 & 1 \\
\hline Constraint $\mathbf{Q}$ & 0 & 1 \\
\hline Total & 1 & 2 \\
\hline
\end{tabular}

Thus the ordering of the variables for selection as the branching variable is $(\mathrm{x}, \mathrm{v})$. Variable $\mathrm{x}$ is selected as the branching variable.

\section{- Active Constraint Scheme B:}

Rationale: The sum of the absolute values of the coefficients of all variables in an active constraint (constraint's weight) is an inverse measure of the impact that a single variable can have on that constraint. If the weight of an active constraint is large, then an individual variable can only bring a small change to that constraint. The sum of the inverse weights of the active constraints in which a candidate variable occurs should give an overall measure of its impact on the active constraints.

Let $\mathrm{SA}_{\mathrm{A} 1}=$ sum of the absolute values of the coefficients of all variables in active constraint A1.

For active constraint $\mathrm{A} 1$, the weight $\mathrm{WA}_{\mathrm{X} 1 \mathrm{Al}}$ of fractional valued integer variable $\mathrm{X} 1$ having a non-zero coefficient in constraint $\mathrm{Al}$ is calculated as:

$$
\mathrm{WAx} 1 \mathrm{~A} 1=\frac{1}{\mathrm{SAA}}
$$


For active constraint $\mathrm{A} 1$, the weight $\mathrm{WA}_{\mathrm{X} 1 \mathrm{~A} 1}$ of fractional valued integer variable $\mathrm{X} 1$ having zero coefficient in constraint A1 is zero. The total weight of each fractional valued integer variable is the sum of its weight over all of the active constraints.

In the given example, the weight of each fractional valued integer variable in constraints $\mathrm{P}$ and $\mathrm{Q}$ is calculated as follows:

\begin{tabular}{|l|c|c|c|}
\hline & SA & WA $_{\mathbf{v}}$ & WA $_{\mathbf{x}}$ \\
\hline Constraint P & 10 & $(1 / 10)=0.1$ & $(1 / 10)=0.1$ \\
\hline Constraint Q & 4 & 0 & $(1 / 4)=0.25$ \\
\hline Total weight & & 0.1 & 0.35 \\
\hline
\end{tabular}

Thus the ordering of the variables for selection as the branching variable is $(x, v)$. Variable $\mathrm{x}$ is selected as the branching variable.

\section{- Active Constraint Scheme C:}

Rationale: The sum of the absolute values of the coefficients of the integer variables in an active constraint (constraint's integer weight) can be considered as an inverse measure of the impact that a single variable can have on that constraint. If the integer weight of an active constraint is large, then an individual integer variable can only cause a small change to that constraint. The sum of the inverse integer weights of active constraints in which a candidate variable occurs should give a measure of its impact on the active constraints. 
Let $\mathrm{SI}_{\mathrm{A} 1}=$ sum of the absolute values of the coefficients of all integer variables in active constraint $A_{1}$.

For active constraint $\mathrm{Al}$, the weight $\mathrm{WI}_{\mathrm{XIAl}}$ of fractional valued integer variable $\mathrm{X} 1$ having a non-zero coefficient in constraint $\mathrm{Al}$ is calculated as:

$$
\mathrm{WIx} \mid \mathrm{A} 1=\frac{1}{\mathrm{SIA1}_{\mathrm{A}}}
$$

For active constraint $\mathrm{A} 1$, the weight $\mathrm{WI}_{\mathrm{X} 1 \mathrm{Al}}$ of fractional valued integer variable $\mathrm{X} 1$ having zero coefficient in constraint $\mathrm{A} 1$ is zero. The total weight of each fractional valued integer variable is the sum of its weight over all of the active constraints.

In the given example, the weight of each fractional valued integer variable in constraints $\mathrm{P}$ and $\mathrm{Q}$ is calculated as follows:

\begin{tabular}{|l|c|c|c|}
\hline & SI & WI $_{\mathbf{v}}$ & WI $_{\mathbf{x}}$ \\
\hline Constraint P & 10 & $(1 / 10)=0.1$ & $(1 / 10)=0.1$ \\
\hline Constraint Q & 3 & 0 & $(1 / 3)=0.333$ \\
\hline Total weight & & 0.1 & 0.433 \\
\hline
\end{tabular}

Thus the ordering of the variables for selection as the branching variable is $(\mathrm{x}, \mathrm{v})$. Variable $\mathrm{x}$ is selected as the branching variable.

\section{- Active Constraint Scheme D:}

Rationale: At the LP relaxation optimum solution, the active constraints force some 
of the integer variables to fractional value. The impact of such fractional valued integer variables on the active constraints can be considered more important than that of the integer variables having integer value. The sum of the absolute values of the coefficients of the fractional valued integer variables in an active constraint (constraint's fractional integer weight) can be considered as an inverse measure of the impact of a single variable on that constraint. If the fractional integer weight of an active constraint is large then an individual fractional valued integer variable can only bring a small change to that constraint. The sum of the inverse fractional integer weights of active constraints in which a candidate variable occurs should give a measure of its impact on the active constraints.

Let $\mathrm{SF}_{\mathrm{A} 1}=$ Sum of the absolute values of the coefficients of all fractional valued integer variables in active constraint $A_{1}$.

For active constraint $\mathrm{A} 1$, the weight $\mathrm{WF}_{\mathrm{X} 1 \mathrm{~A} 1}$ of fractional valued integer variable $\mathrm{X} 1$ having a non-zero coefficient in constraint A1 is calculated as:

$$
\mathrm{WFXIA1}=\frac{1}{\mathrm{SF}_{\mathrm{A} 1}}
$$

For active constraint $\mathrm{A} 1$, the weight $\mathrm{WF}_{\mathrm{XlA1}}$ of fractional valued integer variable $\mathrm{X} 1$ having zero coefficient in constraint $\mathrm{Al}$ is zero. The total weight of each fractional valued integer variable is the sum of its weight over all of the active constraints.

In the given example, the weight of each fractional valued integer variable in constraints $\mathrm{P}$ and $\mathrm{Q}$ is calculated as follows: 


\begin{tabular}{|l|c|c|c|}
\hline & SF & WF $_{\mathbf{v}}$ & WF $_{\mathbf{x}}$ \\
\hline Constraint P & 9 & $(1 / 9)=0.111$ & $(1 / 9)=0.111$ \\
\hline Constraint Q & 2 & 0 & $(1 / 2)=0.5$ \\
\hline Total weight & & 0.111 & 0.611 \\
\hline
\end{tabular}

Thus the ordering of the variables for selection as the branching variable is $(\mathrm{x}, \mathrm{v})$. Variable $\mathrm{x}$ is selected as the branching variable.

\subsection{Active Constraint Schemes based on the coefficient of the fractional valued integer variables in the active constraints}

How much impact a candidate variable has on an active constraint depends on its coefficient in that constraint. This observation underlies the methods described below.

\section{- Active Constraint Scheme E:}

Rationale: The impact of a candidate variable on a constraint can be considered proportional to the relative size of its absolute coefficient with respect to the absolute coefficients of all variables in that constraint.

For active constraint $\mathrm{A} 1, \mathrm{SA}_{\mathrm{A} 1}$ is calculated as shown in Active Constraint Scheme $\mathrm{B}$. The weight $\left(\mathrm{WCA}_{\mathrm{X} 1 \mathrm{~A} 1}\right)$ of fractional valued integer variable $\mathrm{X} 1$ in constraint $\mathrm{A} 1$ is calculated as: 


$$
\text { WCAx1A1 }=\frac{\left|C_{x 1 A 1}\right|}{\text { SAA1 }}
$$

where $\mathrm{C}_{\mathrm{X} 1 \mathrm{~A} 1}=$ coefficient of variable $\mathrm{X} 1$ in constraint $\mathrm{A} 1$. The total weight of each fractional valued integer variable is the sum of its weight over all of the active constraints.

In the given example, the weight of each fractional valued integer variable in constraints $\mathrm{P}$ and $\mathrm{Q}$ is calculated as follows:

\begin{tabular}{|l|c|c|c|}
\hline & SA & WCA $_{\mathbf{v}}$ & WCA $_{\mathbf{x}}$ \\
\hline Constraint $\mathbb{P}$ & 10 & $(8 / 10)=0.8$ & $(1 / 10)=0.1$ \\
\hline Constraint Q & 4 & 0 & $(2 / 4)=0.5$ \\
\hline Total weight & & 0.8 & 0.6 \\
\hline
\end{tabular}

Thus the ordering of the variables for selection as the branching variable is $(\mathrm{v}, \mathrm{x})$. Variable $\mathrm{v}$ is selected as the branching variable.

- Active Constraint Scheme F:

Rationale: The impact of a candidate variable on a constraint can be considered proportional to the relative size of its absolute coefficient with respect to the absolute coefficients of the integer variables in that constraint.

For active constraint $\mathrm{A} 1, \mathrm{SI}_{\mathrm{Al}}$ is calculated as shown in Active Constraint Scheme C. The weight ( $\mathrm{WCI}_{\mathrm{X} 1 \mathrm{~A} 1}$ ) of fractional valued integer variable $\mathrm{X} 1$ in active constraint Al is calculated as: 


$$
\text { WCIX } 1 \mathrm{~A} 1=\frac{\left|\mathrm{CX}_{\mathrm{1}} \mathrm{A} 1\right|}{\mathrm{SI}_{\mathrm{A} 1}}
$$

where $\mathrm{C}_{\mathrm{X} 1 \mathrm{~A} 1}=$ coefficient of variable $\mathrm{X} 1$ in constraint $\mathrm{A} 1$. The total weight of each fractional valued integer variable is the sum of its weight over all of the active constraints.

In the given example, the weight of each fractional valued integer variable in constraints $\mathrm{P}$ and $\mathrm{Q}$ is calculated as follows:

\begin{tabular}{|l|c|c|c|}
\hline & SI & WCI $_{\mathbf{V}}$ & WCI $_{\mathbf{x}}$ \\
\hline Constraint P & 10 & $(8 / 10)=0.8$ & $(1 / 10)=0.1$ \\
\hline Constraint Q & 3 & 0 & $(2 / 3)=0.667$ \\
\hline Total weight & & 0.8 & 0.767 \\
\hline
\end{tabular}

Thus the ordering of the variables for selection as the branching variable is $(v, x)$. Variable $\mathrm{v}$ is selected as the branching variable.

\section{- Active Constraint Scheme G:}

Rationale: The impact of a candidate variable on a constraint can be considered proportional to the relative size of its absolute coefficient with respect to the absolute coefficients of the fractional valued integer variables in that constraint. 
For active constraint $\mathrm{A} 1, \mathrm{SF}_{\mathrm{A} 1}$ is calculated as shown in Active Constraint Scheme D. The weight $\left(\mathrm{WCF}_{\mathrm{X} 1 \mathrm{~A} 1}\right)$ of fractional valued integer variable $\mathrm{X} 1$ in active constraint A1 is calculated as:

$$
W_{C F}{ }_{1 A 1}=\frac{\left|C X_{1 A 1}\right|}{S F_{A 1}}
$$

where $\mathrm{C}_{\mathrm{X} 1 \mathrm{~A} 1}=$ coefficient of variable $\mathrm{X} 1$ in constraint $\mathrm{A} 1$. The total weight of each fractional valued integer variable is the sum of its weight over all of the active constraints.

In the given example, the weight of each fractional valued integer variable in constraints $\mathrm{P}$ and $\mathrm{Q}$ is calculated as follows:

\begin{tabular}{|l|c|c|c|}
\hline & SF & WCF $_{\mathbf{v}}$ & WCF $_{\mathbf{x}}$ \\
\hline Constraint $\mathbf{P}$ & 9 & $(8 / 9)=0.889$ & $(1 / 9)=0.111$ \\
\hline Constraint Q & 2 & 0 & $(2 / 2)=1$ \\
\hline Total weight & & 0.889 & 1.111 \\
\hline
\end{tabular}

Thus the ordering of the variables for selection as the branching variable is $(x, v)$. Variable $\mathrm{x}$ is selected as the branching variable.

\section{- Active Constraint Scheme H:}

Rationale: Instead of looking for the maximum impact of the branching variable on all active constraints we can look for the maximum impact on a single active constraint. Having most impacted a single active constraint, the LP relaxation optimal 
point will move significantly in the variable space represented by that constraint. This might be advantageous in reaching the integer feasible solution quickly.

For each active constraint, the weight of the fractional valued integer variable is calculated as done in one of the Active Constraint Schemes $B$ to $G$ and the resulting schemes are designated as $\mathrm{H}_{\mathrm{B}}$ to $\mathrm{H}_{\mathrm{G}}$ respectively. The variable having the largest weight in an active constraint is selected as the branching variable.

In the given example, using scheme $\mathrm{H}_{\mathrm{G}}$, the weight of each fractional valued integer variable in constraints $\mathrm{P}$ and $\mathrm{Q}$ is calculated as follows:

\begin{tabular}{|l|c|c|c|}
\hline & $\mathbf{S F}$ & $\mathbf{W C F}_{\mathbf{v}}$ & $\mathbf{W C F}_{\mathbf{x}}$ \\
\hline Constraint $\mathbf{P}$ & 9 & $(8 / 9)=0.889$ & $(1 / 9)=0.111$ \\
\hline Constraint $\mathbf{Q}$ & 2 & 0 & $(2 / 2)=1$ \\
\hline
\end{tabular}

Thus the ordering of the variables for selection as the branching variable is $(\mathrm{x}, \mathrm{v})$. Variable $\mathrm{x}$ is selected as the branching variable as it has the most impact on a single active constraint, in this case constraint $\mathrm{Q}$.

\section{- Active Constraint Scheme I:}

Rationale: Active Constraint Schemes A to $\mathrm{G}$ select a branching variable based on different criteria measuring the impact of the candidate variables on the active constraints of the LP relaxation optimal solution. The candidate variable chosen by most of these schemes should have the most overall impact on the active constraints. 
Looking at Active Constraint Schemes A to G, select the variable that is chosen by the largest number of these schemes. In the case of a tie, the variable selected by Active Constraint Scheme G is selected because Active Constraint Scheme G has generally better performance than the other schemes (see empirical results in Section $8.5)$.

In the given example, variable $x$ is selected by Active Constraint Schemes $A, B, C$, $\mathrm{D}$ and $\mathrm{G}$ and variable $\mathrm{v}$ is selected by Active Constraint Scheme $\mathrm{E}$ and F. Since variable $\mathrm{x}$ is chosen by the most Active Constraint Schemes from $A$ to $G$, it is selected as the branching variable.

\subsection{Active Constraint Schemes based on the dual cost of the active constraints}

It may be advantageous to consider the impact of a branching variable on the active constraints and on the objective function simultaneously. This observation underlies the method described below.

\section{- Active Constraint Scheme J:}

The dual cost of the $i$ th constraint of the LP problem is the amount by which the LP optimal objective function value is improved if the right-hand side of the constraint is increased by 1 (see e.g. [Winston 1995]). It is assumed that there is no change in the basis of the LP optimal solution by changing the right-hand side of the constraint. $A \geq$ constraint will have a non-positive dual cost and a $\leq$ constraint will have a nonnegative dual cost. 
Rationale: The absolute value of the dual cost of an active constraint is a measure of its influence on the LP objective function. Changes in an active constraint having a large absolute dual cost can significantly move the LP optimal point closer to the integer feasible solution. The candidate variable should be given more weight if it occurs in an active constraint that has a large absolute value of the dual cost.

The weight (WDAX1A1) of fractional valued integer variable $\mathrm{X} 1$ in active constraint $A 1$ is calculated as:

$$
\mathrm{WDAx}_{1 \mathrm{~A} 1}=\mathrm{W}_{\mathrm{X} 1 \mathrm{~A} 1} \times \mathrm{DA}_{\mathrm{A} 1}
$$

$\mathrm{W}_{\mathrm{X} 1 \mathrm{~A} 1}$ is the weight of variable $\mathrm{X} 1$ as calculated by any one of the Active Constraint Schemes from $B$ to $G$, which results in different variations of Active Constraint Scheme $J$ (i.e. Active Constraint Scheme $J_{B}$ to $J_{G}$ respectively). $D_{A 1}$ is the absolute value of the dual cost of active constraint A1. The total weight of each fractional valued integer variable is the sum of its weight over all of the active constraints.

In the given example, using scheme $J_{G}$, the weight of each fractional valued integer variable in constraints $\mathrm{P}$ and $\mathrm{Q}$ is calculated as follows:

\begin{tabular}{|l|c|c|c|c|}
\hline & D & SF & WDA $_{\mathbf{v}}$ & WDA $_{\mathbf{x}}$ \\
\hline Constraint P & 0.375 & 9 & $(8 / 9) * 0.375=0.333$ & $(1 / 9) * 0.375=0.042$ \\
\hline Constraint Q & 0.312 & 2 & 0 & $(2 / 2) * 0.312=0.312$ \\
\hline Total weight & & & 0.333 & 0.354 \\
\hline
\end{tabular}

Thus the ordering of the variables for selection as the branching variable is $(\mathrm{x}, \mathrm{v})$. Variable $\mathrm{x}$ is selected as the branching variable. 


\section{Node Selection Schemes Based on Integer Infeasibilities}

Most of the existing node selection schemes (Section 4) select an unsolved node that has a higher potential of finding a good integer feasible solution. Two important attributes that are evaluated for each unsolved node are the closeness to integer feasibility and the estimation/projection of the best objective function value of the integer feasible solution either at that node or at any of its descendents. The goal is to quickly reach an integer feasible solution without spending time on solving "unnecessary" nodes.

Different ways of estimating/projecting the best objective function value of an integer feasible solution at a descendant of a given node, as done by different node selection schemes, are explained in Section 4. Such estimates/projections are used to decide if the branch is worthwhile to explore.

Closeness to integer feasibility is usually estimated based on the depth of the node in the B\&B tree (see DFS method in Section 4.1). The depth of the node is its distance from the root node of the $B \& B$ tree. Generally a node that is far from the root node bas fewer degrees of freedom for the integer variables and is closer to integer feasibility compared to a node that is closer to the root node. So continuing the search deeper into the $B \& B$ tree increases the chances of finding an integer feasible solution faster.

This section describes node selection schemes that use integer infeasibilities in the LP relaxation optimal solution of the parent node in order to estimate the closeness of the unsolved child node to integer feasibility. Such schemes use measures based on the integer infeasibility of the variables and the constraints containing those variables, to select the node to solve next. 
Constraints having a non-zero coefficient for an integer infeasible variable are called integer infeasible constraints. Different node selection schemes based on the integer infeasibilities are explained in the following sections.

\subsection{Minimum number of integer infeasible variables (MNIV)}

The unsolved node with the minimum number of integer infeasible variables in the parent node's LP relaxation optimal solution is selected as the next node to solve. Ties are broken randomly. Measuring infeasibility in this way at the $B \& B$ node is known and documented [ILOG Corporation 1999a] but we are not aware of any literature mentioning use of it in the node selection scheme.

\subsection{Minimum sum of the integer infeasibilities (MSII)}

The integer infeasibility of an integer variable is calculated as follows:

Let $F_{k}=$ fractional part in the value of integer variable $k$ at the LP relaxation optimal solution.

IF $F_{k} \geq 0.5$ THEN

$$
\text { Integer infeasibility of variable } \mathrm{k}=\left(1-\mathrm{F}_{\mathrm{k}}\right)
$$

\section{ELSE}

$$
\text { Integer infeasibility of variable } \mathrm{k}=\mathrm{F}_{\mathrm{k}}
$$

In this scheme, the node with the smallest sum of integer infeasibilities over all integer variables is considered closest to an integer feasible solution. Consider, for example, two solved nodes (N1 and N2) having LP relaxation solutions at $(1.5,2.5,1.9)$ and $(1.7,1.7$, 1.2) respectively. Assuming all variables are integer variables, the sum of the integer 
infeasibilities for node $\mathrm{N} 1$ is $(0.5+0.5+0.1=) 1.1$ and the sum of integer infeasibilities for node $\mathrm{N} 2$ is $(0.3+0.3+0.2 \Rightarrow) 0.8$. So node $\mathrm{N} 2$ is considered closer to an integer feasible solution than node N1.

This scheme selects an unsolved node with the minimum sum of integer infeasibilities in the parent node's LP relaxation solution as the next node to solve. Ties are broken randomly. Measuring infeasibility in this way at the $B \& B$ node is known and documented [ILOG Corporation 1999a] but we are not aware of any literature mentioning use of it in the node selection scheme.

\subsection{Minimum number of constraints containing integer infeasible variables (MNIC)}

This scheme estimates integer infeasibilities using constraints. The unsolved node with the minimum number of integer infeasible constraints in its parent node's LP relaxation optimal solution is selected as the next node to solve. The bounding constraints on the integer infeasible variables are not considered while calculating the number of integer infeasible constraints. Ties are broken randomly.

\subsection{Minimum ratio of number of integer infeasible active constraint to total number of active constraints (MNIA)}

As explained in Section 6, the active constraints have more influence on the LP relaxation optimal solution than the inactive ones, so the active constraints might also be considered to have more influence on the integer infeasibilities of the variables. For each unsolved node, the number of active constraints that contain at least one integer infeasible variable 
in the parent node's LP relaxation optimal solution is calculated. This value is normalized using the total number of active constraints. The unsolved node with the minimum ratio is selected as the next node to solve. Ties are broken randomly. 


\section{Empirical Results}

\subsection{Test Environment}

The experiments were carried out on a PC with Microsoft Windows NT 4.0 Operating System and the following characteristics:

- CPU Speed: $266 \mathrm{MHz}$ (Pentium II processor).

- Physical Memory: $288 \mathrm{MB}$

- Free Disk Space: between 1.0 and $1.3 \mathrm{~GB}$

While conducting the experiments, no other applications were running on the machine for the majority of the time. For a small fraction of time, the machine was lightly/medium loaded (in terms of CPU cycle and memory) by other applications. As discussed later, the runtime is not a major factor in comparing the results and so exact data on the machine load is not important and should not affect the results comparison.

\subsection{Test sets}

For most of the experiments that are explained in this section, the MILP problems of MIPLIB 3.0 library [Bixby et at. 1998] are used. Bixby et al. [1998] made the following statement:

"Since its introduction, MIPLIB has become a standard test set for comparing the performance of mixed integer optimization codes." 
MIIPLIB 3.0 library contains both pure and mixed integer linear programs. Most of these problems are from real-world applications e.g. 10teams is a scheduling problem to allocate the teams to time slots in the English football league, danoint and dano3mip are from telecommunication applications and dano3mip is ATM network layout problem. Eckstein [1994], Bixby et al. [2000], Linderoth and Savelsbergh [1999] had used MLP problems of MIPLIB library to conduct their experiments. For some experiments, the following libraries of MLP problems (listed in Mittelmann [2001]) are also used. These libraries contain problems of different sizes (in terms of the number of variables and constraints). The problems from these libraries were used by Mittelmann [2001] to compare the performance of different commercial MILP solvers.

- FCTP library: Some of the fixed charge transportation instances available at ftp://plato.la.asu.edu/pub/fctp/.

- MIP Benchmark library: MILP instances at Argonne national laboratory, Mathematics and Computer Science division. ftp://fp.mcs.anl.gov/neos/mip-bench/.

- MP Test Model library: MLP instances at ftp:/plato.la.asu.edu/pub/milp/. The characteristics of the MILP problems used are briefly described in Appendix A.

\subsection{MILP solvers and parameter settings}

The results obtained by solving the MILP problems using the Active Constraint Schemes of Section 6 and the node selection schemes of Section 7 (plus DFS) are compared with the results obtained using the CPLEX 6.5 MLP solver and the OSLMSLV 3.0 MILP solver. MILP solvers based on the Active Constraint Schemes will be referred to as Active Constraint Solvers. The CPLEX MILP solver is used as a baseline comparison because it 
has better performance, in terms of solution time, on a variety of test libraries compared to other commercial MILP solvers (see benchmark testing by Mittelmann [2001]). The OSLMSLV 3.0 solver is a mature MILP solver that is rich in features and is available free (at the time of writing this) for research use in academic institutions.

Preprocessing, Cut generation and Node heuristic techniques of CPLEX 6.5 and OSLMSLV 3.0 MILP solver are turned off during the experiments. Generally these techniques will be implemented differently in the commercial MILP solvers and by turning them off during the experiments we create a level playing field for these solvers. Note that the Preprocessing, Cut generation and Node heuristics techniques can later be turned back ON for use with the best branching variable and node selection schemes. We recognize the interaction of these features of the MILP solvers with the proposed branching variable selection and node selection schemes but in order to concentrate only on the effectiveness of the selection schemes, the study of such interaction effects has been left as possible future work.

\subsubsection{CPLEX 6.5 MILP solver}

The CPLEX 6.5 MILP solver functionality and parameters are discussed in the CPLEX 6.5 Reference Manual [ILOG Corporation 1999b] and the CPLEX 6.5 User's Manual [ILOG Corporation 1999c]. A C++ prototype program calls the CPLEX 6.5 callable library routine to set the solver parameters, solve the MILP problem and retrieve the result. Parameter settings are as follows. The name shown inside the brackets represents the corresponding CPLEX 6.5 parameter which is explained in the CPLEX 6.5 Reference Manual [ILOG Corporation 1999b]. 
- Branching variable selection scheme (CPX_PARAM_VARSEL): CPLEX Default.

- Node selection scheme (CPX_PARAM_NODESEL): CPLEX Default. This scheme is similar to a Depth First Search strategy until the first feasible solution is found and is then closer to a Best First Search.

- Pre-solving (CPX_PARAM_PREIND): OFF

- Aggregation (CPX_PARAM_AGGIND): OFF

- Root Node Heuristic (CPX_PARAM_HEURISTIC): OFF

- Internal Node Heuristic (CPX_PARAM_HEURFREQ): OFF

- Cut generation (CPX_PARAM_CLIQUES, CPX_PARAM_COVERS, CPX_PARAM_GUBCOVERS, CPX_PARAM_FLOWCOVERS, CPX_PARAM_IMPLBD): OFF

- B\&B Node Limit (CPX_PARAM_NODELIM): explained with the tests.

- Time Limit (CPX_PARAM_TILIM): explained with the tests.

- Tree Memory Size Limit (CPX_PARAM_TRELIM): 128 MB.

- Node File Size Limit (CPX_PARAM_NODEFILELIM): 800 MB.

- Compress Node File (CPX_PARAM_NODEFILEIND): Node file saved on disk and compressed.

- Logging information in file.

- Logging frequency (CPX_PARAM_MIPINTERVAL): 500 nodes

- All other parameters are set at their default values. 


\subsubsection{OSLMSLV 3.0 MILP Solver}

The OSLMSLV 3.0 MILP Solver is a stand-alone program that can be executed from the command line (e.g. DOS prompt) for solving MLP problems. Parameter setting is done using command line options. It understands MPS format as input and in default mode the solver output is printed on the standard output. The following parameters are set for the OSLMSLV 3.0 MILP solver. Names shown in brackets represent the command line option name and are explained in the User Guide [IBM Corporation 1997].

- Branching variable selection scheme: OSLMSLV Default. Based on the estimation of degradation in the objective function value using pseudo-costs.

- Node selection scheme: OSLMSLV Default. This is Depth First Search until the first feasible solution is found and then it is based on the objective function value of the LP relaxation optimal solution and its estimated degradation calculated using pseudocosts.

- Work area limit (dspace): 16000000

- Presolving (presolve and mpretype): OFF

- Heuristics passes (heurpass): 0

- Output Messages (msglevel): Verbose

- B\&B Node Limit (maxnodes): As explained with the tests.

- Time limit (maxtime): As explained with the tests.

- All other parameters are set at their default values. 


\subsubsection{Active Constraint Solvers}

The Active Constraint Solvers are based on the CPLEX 6.5 MILP solver with the CPLEX variable selection and node selection schemes replaced by various Active Constraint schemes and the proposed node selection schemes (plus DFS). A prototype developed in $\mathrm{C}++$ solves the MILP problems using both the original CPLEX 6.5 and the Active Constraint Solvers and compares their results (Appendix I). In addition to using the CPLEX 6.5 callable library routines, this prototype program uses the branching variable selection callback, the node selection callback and other advanced routines in the CPLEX 6.5 callable library [ILOG Corporation 1999a] to implement the Active Constraint Schemes described in Section 6 and the node selection schemes described in Section 7. The user friendly Graphical User Interface (GUT) allows the selection of different Active Constraint Schemes and node selection schemes which leads to different Active Constraint Solvers as shown in Table 1, Table 2 and Table 8. A snapshot of this GUI is shown in Appendix I.

The node selection schemes of Section 7 need to access the parent node LP relaxation optimal solution for each unsolved node. Not all of the needed information on the parent node LP relaxation optimal solution is available via the CPLEX node selection callback. The number of integer infeasibilities and the sum of integer infeasibilities for the unsolved nodes required by MNIV and MSII node selection schemes are available in the node selection callback, but the data required by other node selection schemes in the node selection callback function are not available using any of the CPLEX 6.5 MILP solver routines. For such node selection schemes, the necessary data are saved during execution of the branch variable selection callback and accessed in the node selection 
callback. Mapping of the B\&B nodes to the saved data is based on the node sequence number that is uniquely allocated by the CPLEX 6.5 MLP solver to each node of the $\mathrm{B} \& \mathrm{~B}$ tree.

The parameter settings of the CPLEX 6.5 MILP solver, as explained in Section 8.3.1, are also applied here with a few exceptions:

- Branching variable selection scheme: As set by the user in the GUI. One of the following: Active Constraint Schemes A, B, C, D, E, F, G, $\mathrm{H}_{\mathrm{G}}$, I or $\mathrm{J}_{\mathrm{G}}$

- Node selection scheme: As set by the user in the GUI. One of the following: DFS, MNIV, MSII, MNIC or MNIA.

- B\&B Node Limit (CPX_PARAM_NODELIM): As explained in the tests.

- Time Limit (CPX_PARAM_TILIM): As explained in the tests.

\subsubsection{Designation of the Active Constraint Solvers}

In the following Sections, the designation of the Active Constraint Solver is based on the Active Constraint Scheme and the node selection scheme that it uses. For example, the Active Constraint Solver that uses Active Constraint Scheme B and DFS node selection scheme is designated as "Active Constraint Solver B/DFS" (also referred to as "B/DFS solver").

\subsection{Results comparison criterion}

Many researchers [Dakin 1965, Tomlin 1969, Benichou et al. 1971, Gauthier and Ribiere 1977, Linderoth and Savelsbergh 1999, Mittelman 2001] have used one or more of the 
following criteria to compare the effectiveness of MILP solvers in finding the feasible/optimal solution of MILP problems:

- Solution time (CPU or clock time in seconds)

- Number of solved nodes (i.e. number of solved LP relaxation problems)

- Total number of simplex iterations over all solved nodes

In this thesis, the comparison of the results from different MLP solvers is based on the number of solved B\&B nodes, which is the number of LP relaxation problems solved. The $B \& B$ tree size, in terms of the number of nodes, is the most influential factor in memory consumption of the solver. Hence the number of solved B\&B nodes is a good measure of the amount of effort (both computational and memory space) required by the MILP solver to solve the MILP problem. The solution time is not used for the comparison because the Active Constraint Solvers have no control over the design of the data structures used to solve the LPs and to store their results. These would normally be customized to reduce the decision time in the Active Constraint Schemes and hence to reduce the overall solution time. The number of simplex iterations is not compared because it depends on the LP solver used. The LP solver used by the CPLEX MILP solver and the Active Constraint Solvers is the same but it is different than the one used by the OSLMSLV MILP solver.

\subsection{First Feasible Solution}

In a feasibility experiment we compare a single Active Constraint Solver to CPLEX 6.5 and/or OSLMSLV 3.0 solvers. We perform several feasibility experiments and calculate the value of the following success parameters for each solver. These parameters are then 
compared to evaluate the performance of different solvers for finding the first feasible solution of the MLP problems.

\subsubsection{Definition of Success Parameters}

Various success parameters (underlined) are described below.

Feasibility Success Ratio (FSR): The MILP solver that finds the first feasible solution of the MILP problem by solving the fewest B\&B nodes as compared to other MILP solver(s) is considered faster. Ties are allowed. The FSR of MLP solver A is defined as:

Number of MILP problems for which solver A finds the first feasible

$$
F S R_{A}=\frac{\text { solution at least as fast as other solvers being compared }}{\text { Total number of MILP problems that are comparable }}
$$

In a feasibility experiment, if all solvers find the feasible solution of a MILP problem within the specified limits (e.g. time limit) then we can determine the fastest solver(s) among them for this MILP problem (results are comparable). If one or more solvers are unable to find the feasible solution within the specified limits then we consider such results comparable if and only if:

- At least one solver (say solver A), among the solvers being compared, has found the feasible solution of this MILP problem and

- For each other solver (say solver B) that did not find the feasible solution of this MILP problem, the number of nodes solved by solver $\mathrm{A} \leq$ the number of nodes solved by solver B, plus 1 . 
The un-comparable results are not included in the FSR calculation of the solvers (also see Note 1 on page 87 ).

Feasibility Success Factor (FSF): To get an idea of how much faster a solver is than others (as opposed to how frequently it is faster) we define FSF as follows. In a feasibility experiment, the FSF of MILP solver $A$ in finding the first feasible solution of MILP problem $B$ is calculated as:

Maximum number of $B \& B$ nodes solved by any solver, among the solvers $F S F_{A B}=\frac{\text { being compared, to find the first feasible solution of problem } B}{\text { Number of } B \& B \text { nodes solved by solver } A \text { to find the first feasible solution }}$ of problem $B$

Note that $\mathrm{FSF}_{\mathrm{AB}} \geq 1$. The overall FSF of solver $\mathrm{A}$ (i.e. $\mathrm{FSF}_{\mathrm{A}}$ ) is the average of its FSF values for all MILP problems. If one or more solvers are unable to find the feasible solution of a MILP problem within the specified limits then we cannot determine the numerator term in the above equation. Hence we do not consider such MILP problems while calculating the FSF of the solvers (also see Note 1 on page 87). A large value of FSF is considered better for the solver. The FSF value of a solver should always be considered relative to the FSF value(s) of the solver(s) it is compared against in order to decide if the solver is faster/slower than others. Consider for example two solvers, $X$ and $\mathrm{Y}$, used to find the feasible solution of MILP problems $\mathrm{P}$ and $\mathrm{Q}$. X solves 10 and 80 nodes to find the feasible solution of problems $\mathrm{P}$ and $\mathrm{Q}$ respectively. $\mathrm{Y}$ solves 100 and 20 nodes to find the feasible solution of problems $\mathrm{P}$ and $\mathrm{Q}$ respectively.

$$
\mathrm{FSF}_{\mathrm{X}}=(100 / 10+80 / 80) / 2=5.5
$$




$$
\mathrm{FSF}_{\mathrm{Y}}=(100 / 100+80 / 20) / 2=2.5
$$

The FSF indicate that $\mathrm{X}$ is, on average, faster than $\mathrm{Y}$.

Feasibility Success Factor Prime (FSF'): To find out how much faster a solver is than others for the MLP problems for which it is faster/equally as fast (as opposed to all MULP problems in FSF) we define FSF' as follows. The FSF' of MILP solver A (i.e. FSF' ${ }_{\mathrm{A}}$ ) is the average of its FSF values for the MILP problems for which it is faster/equal as fast as the other solvers. In the above example, the FSF' of $\mathrm{X}$ and $\mathrm{Y}$ solvers are:

$$
\begin{aligned}
& \text { FSF'X }_{X}=(100 / 10) / 1=10 \\
& \text { FSF'Y }_{Y}=(80 / 20) / 1=4
\end{aligned}
$$

The calculated FSF' can be interpreted as follows. If solver $\mathrm{X}$ is faster/equally as fast then it is on average 10 times faster than $\mathrm{Y}$, and if solver $\mathrm{Y}$ is faster/equally as fast then it is on average 4 times faster than X. Similar to the condition used for the FSF calculation, we do not consider those MILP problems for the FSF' calculation whose feasible solution is not found by at least one solver (also see Note 1 on page 87). A large value of FSF' is considered better for the solver.

Quality Success Ratio (QSR): The optimality gap at the first integer feasible solution is used to compare the quality of the integer feasible solutions found by different MILP solvers. The optimality gap of an integer feasible solution is explained in Section 2.1.1. We calculate the QSR of MILP solver A as follow: 
Number of MILP problems for which solver A finds the first feasible solution of better / equal optimality gap compared to other solvers

$$
\operatorname{QSR}_{A}=\frac{\text { being compared }}{\text { Total number of MILP problems }}
$$

Quality Success Ratio Prime (OSR'): In order to determine if the solver finds the feasible solution faster at the cost of degrading the quality of the solution we define QSR'. The QSR' of the MILP solver measures the quality of the feasible solutions that are found faster by that solver.

$$
\begin{aligned}
& \begin{array}{l}
\text { Number of MILP problems for which solver } A \text { finds the first feasible } \\
\text { solution at least as fast as other solvers being compared } \\
\text { and has better / equal optimality gap at the feasible solution } \\
\text { compared to at least one of other solvers }
\end{array} \\
& \begin{array}{l}
\text { Total number of MILP problems for which solver A finds the first feasible } \\
\text { solution at least as fast as other solvers being compared }
\end{array}
\end{aligned}
$$

Similar to the condition used for the FSF and FSF' calculations, we do not consider those MILP problems in the QSR and QSR' calculation whose feasible solution is not found by at least one solver being compared (also see Note 1 below).

Note 1: We recognize that by ignoring the un-comparable results in the FSR calculation of the solvers, the resulting FSR may be biased towards a particular solver. Similarly, while calculating the FSF, FSF', QSR and QSR' of the solvers we ignore the problems that are unsolved by one or more solvers and this may cause these parameters to be biased towards a particular solver. Un-comparable results are documented while describing the 
results in the following Sections so that we get some idea of the amount of biasing that is possible.

\subsubsection{Evaluating the Active Constraint Schemes}

This Section describes the feasibility experiments for comparing the Active Constraint Solvers based on different Active Constraint Schemes individually to the CPLEX 6.5 MILP solver (baseline solver). These experiments compare the feasible solutions obtained using an Active Constraint Solver and the CPLEX 6.5 MLP solver, allowing us to calculate various success parameters (e.g. FSR) for each Active Constraint Solver. The success parameters allow us to directly evaluate the performance of different Active Constraint Solvers against the CPLEX 6.5 MILP baseline solver. The Active Constraint Solver that most outperforms CPLEX 6.5 is the best solver. The best Active Constraint Solver will be used in the later experiments. In these experiments, the DFS node selection strategy, which is usually considered effective in finding the integer feasible solution faster, is used by all of the Active Constraint Solvers. Different node selection strategies are considered later. As shown later in the empirical results, Scheme G performs better among Schemes A to $\mathrm{G}$ and so Schemes $\mathrm{H}$ and J use the weight calculation of Scheme $\mathrm{G}$ to determine the weight of each fractional valued integer variable in the active constraints.

The CPLEX 6.5 MILP solver is initially used to find the first feasible solution of the MILP problems of MIPLIB 3.0 library (65 MILP problems). The time limit is set to 40,000 seconds and no limit is set for the number of $B \& B$ nodes. The Active Constraint 
Solvers are then used to solve the same MILP problems. No time limit is set but the node limit is set to:

The number of B\&B nodes solved by CPLEX 6.5 MLP solver to find the first integer feasible solution +1000

The node limit is set in this way for the Active Constraint Solvers because there are many Active Constraint Solvers and MILP problems to solve and so we want to limit the amount of time spent in conducting these experiments. Also in these experiments we are mainly interested in knowing if the Active Constraint Solver requires fewer B\&B node solutions compared to the CPLEX 6.5 MILP solver for finding the first integer feasible solution (i.e. results are comparable).

The FSR, FSF, FSF', QSR and QSR' are calculated by comparing the feasible solutions obtained using each Active Constraint Solver to that of the CPLEX 6.5 MILP solver. The detailed comparison of the results obtained by different solvers is shown in Appendix B. The success parameter values for the Active Constraint Solvers are shown in Table 1.

Note 2 (related to FSR in Table 1): The CPLEX 6.5 solver is unable to find the feasible solution of the dano3mip problem within the time limit. According to the condition on comparable results for the FSR calculation, the dano3mip problem is marked uncomparable for F/DFS and $J_{G} /$ DFS solvers. All other problems of MIPLIB 3.0 library are comparable. 
Note 3 (rellated to FSF and QSR in Table 1): The dano3mip problem is not included in the FSF and QSR calculations for all Active Constraint Solvers. In addition, the following problems of MIPLIB 3.0 library are also ignored during the FSF and QSR calculation of a particular Active Constraint Solver as their feasible solutions are not found by that solver within the specified node limits. All other problems of MIPLIB 3.0 library are considered in the FSF and QSR calculations.

- A/DFS solver: arki001, enigma, harp2, noswot, rout (5 problems)

- B/DFS solver: air04, arki001, blend2, enigma, noswot (5 problems)

- C/DFS solver: air04, arki001, blend2, danoint, enigma, noswot (6 problems)

- D/DFS solver: arki001, danoint, enigma, flugpl, harp2, noswot (6 problems)

- E/DFS solver: air04, arki001, blend2, cap6000, harp2 (5 problems)

- F/DFS solver: air04, arki001, cap6000, harp2 (4 problems)

- G/DFS solver: arki001, cap6000, flugpl, harp2, 1seu (5 problems)

- $\mathrm{H}_{\mathrm{G}}$ /DFS solver: arki001, cap6000, harp2, 1seu (4 problems)

- I/DFS solver: air04, blend2, cap6000, flugpl, noswot (5 problems)

- $\mathrm{J}_{\mathrm{G}} / \mathrm{DFS}$ solver: arki001, enigma, flugpl, harp2, mkc, noswot, rout (7 problems)

Note that in worst case only $(7+1) / 65(=12.3 \%)$ of problems in the test set are eliminated from the FSF and QSR calculations.

Note 4 (related to FSF' and OSR' in Table 1): Only the dano3mip problem is not included in the FSF' and QSR' calculation of all Active Constraint Solvers. 


\begin{tabular}{|c|c|c|c|c|c|}
\hline $\begin{array}{c}\text { Active } \\
\text { Constraint } \\
\text { Solver }\end{array}$ & $*$ FSR & ${ }^{* *}$ FSF $/$ FSF CPLEX $_{\text {I }}$ & ${ }^{* * * * *}$ FSF & ${ }^{* * *}$ QSR & ${ }^{* * * *}$ QSR \\
\hline $\mathrm{A} / \mathrm{DFS}$ & 0.68 & $\begin{array}{ll}3 & 11.50_{11.65}\end{array}$ & $\begin{array}{ll}2 & 15.40\end{array}$ & 0.42 & 0.51 \\
\hline B/DFS & 0.66 & $9.95 / 1.40$ & 13.58 & 0.39 & 0.48 \\
\hline C/DFS & 0.66 & $10.11_{1 / 41}$ & 13.58 & 0.41 & 0.50 \\
\hline $\mathrm{D} / \mathrm{DFS}$ & 0.63 & $\begin{array}{ll}1 & 11.76_{/ 1.33}\end{array}$ & $\begin{array}{ll}1 & 16.60\end{array}$ & $\begin{array}{ll}3 & 0.47\end{array}$ & $\begin{array}{ll}3 & 0.57\end{array}$ \\
\hline E/DFS & $\begin{array}{ll}3 & 0.69\end{array}$ & $10.77_{/ 1.50}$ & 14.10 & 0.44 & 0.50 \\
\hline F/DFS & $\begin{array}{ll}2 & 0.70\end{array}$ & $10.84_{/ 1.49}$ & 14.12 & 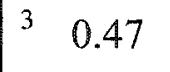 & 0.53 \\
\hline G/DFS & $\begin{array}{ll}3 & 0.69\end{array}$ & $\begin{array}{ll}2 & 11.61_{/ 1.41}\end{array}$ & $\begin{array}{ll}3 & 15.23\end{array}$ & $\begin{array}{ll}1 & 0.53\end{array}$ & $\begin{array}{ll}1 & 0.61\end{array}$ \\
\hline $\mathrm{H}_{\mathrm{G}} / \mathrm{DFS}$ & $\begin{array}{ll}3 & 0.69 \\
\end{array}$ & $11.04 / 1.67$ & 14.70 & \begin{tabular}{|ll}
2 & 0.48 \\
\end{tabular} & $\begin{array}{ll}2 & 0.59 \\
\end{array}$ \\
\hline IDFS & $\begin{array}{ll}1 & 0.74\end{array}$ & $10.55_{/ 1.21}$ & 12.98 & $\begin{array}{ll}3 & 0.47\end{array}$ & 0.53 \\
\hline $\mathrm{J}_{\mathrm{G}} / \mathrm{DFS}$ & 0.67 & $11.47_{/ 2.19}$ & 14.88 & \begin{tabular}{|ll}
3 & 0.47 \\
\end{tabular} & 0.53 \\
\hline
\end{tabular}

Table 1: FSR, FSF, FSF', QSR and QSR' of the Active Constraint Solvers compared individually with CPLEX 6.5 MILP solver over the MILP problems of MIPLIB 3.0 library.

* See Note 2 on page 89

** The FSF values are biased towards the Active Constraint Solvers. See the FSF remark below.

*** See Note 3 on page 90 . Also see QSR remark below.

$* * * *$ See Note 4 on page 90

The following remarks are made based on the results shown in Table 1 (and Appendix B):

- The FSR are all in favor of the active constraint methods. This shows the value of considering the active constraints rather than objective function measures in finding the first feasible solution of MILP problems faster. 
- The FSF' shows that the average speedups achieved using active constraint methods when they find the first integer feasible solution faster are very dramatic.

- As the FSF of the solver should be considered relative to the FSF of the solver(s) it is compared against, we have also shown the FSF values of the CPLEX 6.5 solver in the FSF column. Note that a larger number of problems are left unsolved by the Active Constraint Solvers (ranging from 4 to 7 as listed in Note 3 on page 90) compared to that of CPLEX 6.5 (only 1 problem). For these problems, the CPLEX 6.5 MILP solver is faster than the Active Constraint Solvers but the results are eliminated from the FSF calculation for the reason explained in Section 8.5.1. Hence the FSF shown in the table are biased toward the Active Constraint Solver. However less than $11 \%$ of the problems are biased towards the Active Constraint Solver in the worst case.

- Similar to the FSF, the listed problems are also ignored for the QSR calculations for the Active Constraint Solvers. However unlike FSF, we do not believe that the QSR are biased towards the Active Constraint Solvers because we cannot predict the quality of the feasible solutions that might be found by the Active Constraint Solvers. The QSR for most of the Active Constraint Solvers are less than but close to 0.5. Hence compared to the Active Constraint Solvers, the CPLEX 6.5 MILP solver generally finds better quality feasible solutions for a few more problems.

- The QSR' shows that the quality of the first integer feasible solution that are found faster using active constraint methods is generally improved or at least not worsened.

- As shown in Table 1, the Active Constraint Solvers I/DFS, D/DFS, D/DFS, G/DFS and G/DFS lead the FSR, FSF, FSF', QSR and QSR' calculations respectively. The performance of D/DFS, F/DFS, G/DFS, and I/DFS solvers are close to each other. 
However we have selected G/DFS solver as the best solver among them for the following reasons:

$\circ$ G/DFS solver is $1^{\text {st }}, 1^{\text {st }}, 2^{\text {nd }}, 3^{\text {rd }}$ and $3^{\text {rd }}$ in the QSR, QSR', FSF, FSF' and FSR calculations respectively. It finds the first feasible solution faster/equally as fast as the CPLEX 6.5 MILP solver for $69 \%$ of the MULP problems and on average it finds these solutions 15.23 times faster. Also the qualities of the first feasible solutions that are found faster/equally as fast are better/equal to those of the CPLEX 6.5 MILP solver $61 \%$ of the time.

- The FSF' (and FSF) of the D/DFS solver is greater than that of the G/DFS solver. However its FSR, QSR and QSR' are smaller than that of the G/DFS solver. Being conservative, we consider that the number of faster solutions (FSR) and the quality of the feasible solutions (QSR and QSR') are more important than the average speedups.

- The FSR of the I/DFS solver is greater than that of the G/DFS solver but its FSF', QSR and QSR' are smaller. Also the voting method (Active Constraint Scheme I), which is used in I/DFS solver, requires significantly more computation for selecting the branching variable compared to Active Constraint Scheme G which is used by the G/DFS solver, and only marginally improves FSR over the G/DFS solver.

- The FSR of the F/DFS solver is slightly greater than that of the G/DFS solver but its FSF', QSR and QSR' are considerably smaller than that of the G/DFS solver.

- For some MLP problems, Active Constraint Solver G/DFS performs significantly better than the CPLEX 6.5 MILP solver. For example, the G/DFS solver found the 
first feasible solution of the 10 teams, enigma, fast0507 and swath problems after solving $661,545,43$ and 27 B\&B nodes respectively while CPLEX 6.5 needs 7814 , 5975,2404 and $6206 \mathrm{~B} \& \mathrm{~B}$ nodes respectively to find the first feasible solution for the same problems. Another example is dano3mip for which CPLEX reached the time limit of 40,000 seconds ( 11.11 hours) without a feasible solution while G/DFS solver was able to find a feasible solution for that problem within that time limit. On the other hand, G/DFS solver needs to solve at least 1000 nodes more than CPLEX 6.5 for the arki001, cap6000, flugpl, harp2 and 1seu problems.

\subsubsection{Evaluating the Node Selection Schemes}

Table 1 shows the results when using the DFS node selection scheme. The results obtained using Active Constraint Scheme G and different node selection schemes are compared to the CPLEX 6.5 MLP solver in order to find the node selection scheme that along with Active Constraint Scheme G is fastest at finding the first feasible solution of the MILP problems. Other than DFS, various node selection schemes based on integer infeasibilities (explained in Section 7) are used. The resulting Active Constraint Solvers are listed in Table 2. These solvers are compared individually to the CPLEX 6.5 MILP solver.

The CPLEX 6.5 MILP solver is initially used to find the first feasible solution of the MLP problems of the MIPLIB 3.0 library (65 problems). The time limit is set to 40,000 seconds. The Active Constraint Solvers shown in Table 2 are then used to solve the same problems. The time limit is set to 40,000 seconds for G/DFS, G/MNIV and G/MSII solvers and 50,000 or more for the remaining solvers. Since the node selection 
callback function performs computations for each candidate node, the time limit is mainly set to prevent spending an excessive amount of time on any problem. Active Constraint Solvers G/DFS, G/MNIV and G/MSII get all required information directly from the CPLEX interface for making the decision in the node selection routine and so the time limit for them is set to a lower value compared to solvers G/MNIC and G/MNIA. The idea here is to decrease the possibility of exceeding the time limit while solving any problem (i.e. the solver should terminate at a feasible solution) and at the same time to not spend an excessive amount of time on any single problem. This will give a good number of comparable results in a reasonable amount of time. The B\&B node limit is set to:

The number of B\&B nodes solved by CPLEX 6.5 MILP solver to find the first integer feasible solution +1000

The FSR, FSF, FSF', QSR and QSR' are calculated by comparing the feasible solutions obtained using each Active Constraint Solver to that of the CPLEX 6.5 MILP solver. The detailed comparison of the results is shown in Appendix C. The success parameter values for the Active Constraint Solvers are shown in Table 2.

Note 5 (related to FSR in Table 2): For the dano3mip problem, the CPLEX 6.5, G/MNIV, G/MSII and G/MNIC solvers are unable to find the feasible solution within the time/node limit. According to the condition on the comparable results, the dano3mip problem is marked un-comparable for Active Constraint Solvers G/MNIV, G/MSII, G/MNIC and G/MNIA. The G/MNIC and G/MNIA solvers terminated abnormally while 
solving the enigma and harp2 problems and so they were also marked un-comparable for these solvers. All other problems in the MIPLIB 3.0 library are comparable.

Note 6 (related to FSF and QSR in Table 2): The dano3mip problem is not included in the FSF and QSR calculation of all Active Constraint Solvers. In addition, the following problems from the MIPLIB 3.0 library are also ignored during the FSF and QSR calculations of a particular Active Constraint Solver as their feasible solutions are not found by that solver within the specified limits.

- G/DFS solver: arki001, cap6000, flugpl, harp2, lseu (5 problems)

- G/MNIV solver: cap6000, dano3mip, harp2 (3 problems)

- G/MSII solver: arki001, blend2, cap6000, dano3mip, flugpl, harp2, p0548 (7 problems)

- G/MNIC solver: arki001, bell5, dano3mip, enigma, fiber, harp2, p0548, pk1 (8 problems). The solver terminated abnormally for the enigma and harp2 problems.

- G/MNIA solver: arki001, bell5, cap6000, fiber, flugpl, enigma, harp2, p0548 (8 problems). The solver terminated abnormally for the enigma and harp2 problems.

Note 7 (related to FSR' and QSR' in Table 2): The dano3mip problem is not included in the FSF' and QSR' calculations of all Active Constraint Solvers. Also enigma and harp2 are left out of the FSF' and QSR' calculations of the G/MNIC and G/MNIA solvers. All other problems are included in the FSF' and QSR' calculations. 


\begin{tabular}{|c|c|c|c|c|c|}
\hline $\begin{array}{l}\text { Active } \\
\text { Constraint } \\
\text { Solver }\end{array}$ & ${ }^{*}$ FSR & ${ }^{* *}$ FSF/FSF CPLEX & ${ }^{* * * * *}$ FSF & ${ }^{* * *}$ QSR & ${ }^{* * * * *}$ QSR' $^{\prime}$ \\
\hline G/DFS & 10.69 & $1 \quad 11.61_{/ 1.41}$ & $2 \quad 15.23$ & ${ }^{1} 0.53$ & 30.61 \\
\hline G/MNIV & ${ }^{2} \quad 0.61$ & $9.42_{22.38}$ & ${ }^{3} 14.39$ & $\begin{array}{ll}3 & 0.47\end{array}$ & 0.59 \\
\hline G/MSII & 0.53 & ${ }^{2} \quad 10.16_{11.80}$ & 16.62 & ${ }^{1} \quad 0.53$ & 0.59 \\
\hline G/MNIC & 0.58 & $7.21_{/ 1.42}$ & 10.83 & ${ }^{1} \quad 0.53$ & ${ }^{1} \quad 0.69$ \\
\hline G/MNIA & ${ }^{3} 0.60$ & $7.61_{1 / .93}$ & 11.01 & 20.50 & ${ }^{2} 0.62$ \\
\hline
\end{tabular}

Table 2: FSR, FSF, FSF', QSR and QSR' of Active Constraint Solvers using different node selection schemes compared individually with the CPLEX 6.5 MILP solver over the MILP problems of the MIPLIB 3.0 library.

* See Note 5 on page 95

** The FSF values are biased towards Active Constraint Solvers. See the FSF remark below.

*** See Note 6 on page 96

**** See Note 7 on page 96

The following remarks are made based on the results shown in Table 2:

- The DFS node selection strategy along with Active Constraint Scheme G finds the first feasible solution faster for the largest number of MILP problems compared to other node selection schemes that are based on integer infeasibilities in the LP relaxation optimal solution.

- The FSF' shows that the speed-up obtained using the DFS node selection scheme is better than most of the other node selection schemes.

- The QSR' of the G/MNIC and G/MNIA solvers are greater than the QSR' of the G/DFS solver but their FSR and FSF' are far smaller that those of G/DFS solver. 
- As the FSF of the solver should be considered relative to the FSF of the solver(s) it is compared against, we have also shown the FSF values of the CPLEX 6.5 solver in the FSF column. Note that larger numbers of problems are left unsolved by the Active Constraint Solvers (ranging from 3 to 8 as listed above) compared to that of CPLEX 6.5 (only 1 problem). For these problems (except enigma and harp2 which terminated abnormally for the G/MNIC and G/MNIA solver) the CPLEX 6.5 MILP solver is faster than the Active Constraint Solvers but we have to eliminate them from the FSF calculation for the reason explained in Section 8.5.1. Hence the FSF shown in the table are biased towards the Active Constraint Solver. However less than $11 \%$ of the problems are biased towards the Active Constraint Solver in the worst case.

- The QSR of G/DFS, G/MSII and G/MNIC solvers are the best among the Active Constraint Solvers shown in Table 2.

- The MNIC node selection scheme improves QSR and QSR' at expense of FSR.

\subsubsection{Comparison of Active Constraint Solvers G/DFS, CPLEX 6.5 and OSLMSLV 3.0 using different test libraries}

Active Constraint Solver G/DFS is compared with the CPLEX 6.5 and OSLMSLV 3.0 MILP solvers below. The results of all three solvers are compared together. For all solvers, the time limit is set to 40,000 seconds and no limit is set on the B\&B nodes. Table 3 shows the FSR, FSF, FSF', QSR and QSR' of CPLEX 6.5, OSLMSLV 3.0 and Active Constraint Solver G/DFS as calculated by solving the MILP problems of MIPLIB 3.0 library (65 problems). The detailed comparison of the results is given in Appendix D. The FSR and QSR in Table 3 do not sum to 1 because of ties. 
Note 8 (related to FSR in Table 3): All problems are comparable.

Note 9 (related to FSF and OSR in Table 3): The following 8 problems from the MIPLIB 3.0 library are ignored during FSF and QSR calculations of each solver as their feasible solutions are not found within the time limit by one of the solvers.

- CPLEX 6.5 solver: dano3mip (1 problem)

- OSLMSLV 3.0 solver: mitre, p0548 (2 problems)

- G/DFS solver: arki001, cap6000, flugpl, harp2, Iseu (5 problems)

All other problems are considered in the FSF and QSR calculations.

Since more problems ( 5 problems) are left unsolved by the G/DFS solver that are executed faster by other solvers, the FSF is biased towards the G/DFS solver. Among the CPLEX 6.5 and OSLMSLV 3.0 solvers, the FSF is biased towards OSLMSLV 3.0. The QSR values are not biased.

Note 10 (related to FSF' and QSR' in Table 3): The CPLEX 6.5 MLP solver is faster than the G/DFS and OSLMSLV 3.0 solvers for the following 3 problems but these problems are not included in its FSF' and QSR' calculation because the G/DFS solver is unable to find their feasible solution within the time limit.

- G/DFS solver: cap6000, flugpl, harp2 (3 problems)

The OSLMSLV 3.0 solver is faster than the G/DFS and CPLEX 6.5 solvers for the following 2 problems but these are not included in its FSF' and QSR' calculation because the G/DFS solver is not able to find their feasible solutions within the time limit. 
- G/DFS solver: arki001, lseu (2 problems)

The G/DFS solver is faster than the CPLEX 6.5 and OSLMSLV solvers for the following 3 problems but these are not included in its FSF' and QSR' calculation because either CPLEX 6.5 or OSLMSLV are not able to find their feasible solutions within the time limit.

- CPLEX 6.5 solver: dano3mip (1 problem)

- OSLMSLV 3.0 solver: mitre, p0548 (2 problems)

\begin{tabular}{|l|c|c|c|c|c|}
\hline \multicolumn{1}{|c|}{ MILP Solver } & ${ }^{*}$ FSR & ${ }^{* *}$ FSF & $\begin{array}{l}* * * * \\
\text { FSF' }\end{array}$ & ${ }^{* * *}$ QSR & $\begin{array}{l}\text { **** }^{*} \\
\text { QSR }^{\prime}\end{array}$ \\
\hline ILOG CPLEX 6.5 & 0.28 & 2.41 & 4.53 & 0.33 & 0.83 \\
\hline OSLMSLV 3.0 & 0.11 & 8.36 & 63.54 & 0.46 & 0.86 \\
\hline Active Constraint Solver G/DFS & 0.66 & 13.31 & 17.78 & 0.25 & 0.77 \\
\hline
\end{tabular}

Table 3: FSR, FSF, FSF', QSR and QSR' of CPLEX 6.5, OSLMSLV 3.0 and Active Constraint Solver G/DFS over the MILP problems of MIPLIB 3.0 library.

* See Note 8 on page 99

** The FSF values are biased towards G/DFS solver and then towards OSLMSLV 3.0 solver. See Note 9 on page 99 for explanation.

**** See Note 9 on page 99

**** See Note 10 on page 99

Only the MILP problems of MIPLIB 3.0 library are used in Table 3. Next, the CPLEX 6.5, OSLMSLV 3.0 and G/DFS solvers are compared for the MILP problems of the FCTP (14 problems), MIP Benchmark (8 problems) and MP Test Model (15 problems) libraries to check whether Active Constraint Solver G/DFS performs 
consistently well on other test libraries. The time and the node limits for all solvers are similar to those used for the FSR calculations of Table 3. The FSR, FSF and QSR values using the FCTP, MIP Benchmark and MP Test Model libraries are shown in Tables 4, 5 and 6 and the detailed comparisons are shown in Appendices E, F and G respectively.

Note 11 (FSR, FSF, QSR, FSF' and QSR' in Table 4, Table 5 and Table 6): All solvers are able to find the feasible solutions of all problems within the specified limits. Hence none of the problems are excluded from the FSR, FSF, FSF', QSR and QSR' calculations.

\begin{tabular}{|l|c|c|c|c|c|}
\hline \multicolumn{1}{|c|}{ MILP Solver } & FSR & FSF & FSF' & QSR & QSR $^{\prime}$ \\
\hline ILOG CPLEX 6.5 & 0.00 & 1.16 & 0.00 & 0.14 & 0.00 \\
\hline OSLMSLV 3.0 & 0.07 & 1.60 & 8.50 & 0.29 & 1.00 \\
\hline Active Constraint Solver G/DFS & 0.93 & 4.29 & 4.11 & 0.57 & 0.92 \\
\hline
\end{tabular}

Table 4: FSR, FSF, FSF', QSR and QSR' of CPLEX 6.5, OSLMSLV 3.0 and Active Constraint Solver G/DFS over the MILP problems of FCTP library.

See Note 11 on page 101 


\begin{tabular}{|l|c|c|c|c|c|}
\hline \multicolumn{1}{|c|}{ MULP Solver } & FSR & FSF & FSF' & QSR & QSR $^{\prime}$ \\
\hline ILOG CPLEX 6.5 & 0.13 & 1.17 & 1.39 & 0.25 & 1.00 \\
\hline OSLMSLV 3.0 & 0.25 & 7.18 & 14.84 & 0.25 & 1.00 \\
\hline Active Constraint Solver G/DFS & 0.63 & 29.84 & 43.99 & 0.50 & 1.00 \\
\hline
\end{tabular}

Table 5: FSR, FSF, FSF', QSR and QSR' of CPLEX 6.5, OSLMSLV 3.0 and Active Constraint Solver G/DFS over the MILP problems of MIP Benchmark library. See Note 11 on page 101

\begin{tabular}{|l|c|c|c|c|c|}
\hline \multicolumn{1}{|c|}{ MILP Solver } & FSR & FSF & FSF' & QSR & QSR $^{9}$ \\
\hline ILOG CPLEX 6.5 & 0.27 & 7.25 & 3.60 & 0.13 & 0.75 \\
\hline OSLMSLV 3.0 & 0.13 & 15.07 & 45.40 & 0.67 & 1.00 \\
\hline Active Constraint Solver G/DFS & 0.73 & 53.56 & 72.28 & 0.20 & 0.73 \\
\hline
\end{tabular}

Table 6: FSR, FSF, FSF', QSR and QSR' of CPLEX 6.5, OSLMSLV 3.0 and Active Constraint Solver G/DFS over the MILP problems of MP Test Model libraries.

See Note 11 on page 101

The results obtained by all solvers over the MIPLIB 3.0, FCTP, MIP Benchmarks and MP Test Model libraries are combined in order to calculate the combined values of the various success parameters (Table 7) 


\begin{tabular}{|l|c|c|c|c|c|}
\hline \multicolumn{1}{|c|}{ MIILP Solver } & FSR & FSF & FSF & QSR & QSR $^{\prime}$ \\
\hline ILOG CPLEX 6.5 & 0.23 & 2.89 & 4.18 & 0.27 & 0.83 \\
\hline OSLMSLV 3.0 & 0.12 & 8.32 & 44.67 & 0.45 & 0.92 \\
\hline Active Constraint Solver G/DFS & 0.71 & 19.80 & 25.79 & 0.31 & 0.81 \\
\hline
\end{tabular}

Table 7: FSR, FSF, FSF', QSR and QSR' of CPLEX 6.5, OSLMSLV 3.0 and Active

Constraint Solver G/DFS over the combined MILP problems of MIPLIB 3.0, FCTP, MIP Benchmark library, MP Test Model libraries

Notes on Table 3 also apply here.

The following remarks are made based on the results in Tables 3-7:

- The FSR in Table 7 indicate that Active Constraint Solver G/DFS finds the first feasible solution of MILP problems faster/equally as fast as the CPLEX 6.5 and the OSLMSLP 3.0 solvers for $71 \%$ of the problems. For such problems, the G/DFS solver is on average faster by 25.79 times (FSF') than the slowest solver from CPLEX 6.5 and OSLMSLV 3.0. Also for such problems, on average 81\% (QSR') of the time the quality of the feasible solution found by the G/DFS solver is at least not worse than found by the CPLEX 6.5 and OSLMSLV 3.0 solvers. Thus the G/DFS solver performs well on a large portion of the MILP problems from different libraries.

- As explained in Note 9 on page 99 the FSF in Table 7 are biased towards G/DFS solver and then towards OSLMSLV solver. If we ignore such biasing then the FSF in Table 7 indicate that overall Active Constraint Solver G/DFS is significantly faster than CPLEX 6.5 and OSLMSLP 3.0 MILP solver for all test libraries. 
- The QSR in Table 7 indicate that the feasible solutions that are found by OSLMSLV 3.0 solver are generally of better quality than the other two solvers. The G/DFS solver is at the second position and finds at least the same quality feasible solution as the other two solvers for almost $1 / 3^{\text {rd }}$ of the total number of problems.

- The G/DFS solver leads the FSR and FSF calculations in all test libraries (Table 3-6). The CPLEX 6.5 solver is at second place for FSR calculations and OSLMSLV 3.0 is at second place for FSF calculations. This indicates that CPLEX 6.5 performs better on more problems than OSLMSLV 3.0 (FSR) but the latter performs more consistently than the former (i.e. average performance is better). The G/DFS solver outperforms them in both scenarios.

- The OSLMSLV 3.0 solver leads the QSR for MIPLIB 3.0 and MP Test Model libraries. The G/DFS solver leads the QSR for the other two libraries.

\subsection{Optimal / Final Feasible solution}

This section provides experimental results to evaluate the effectiveness of the Active Constraint Scheme for finding the optimal solution of MILP problems faster.

Optimality Success Ratio (OSR): Similar to the conditions used for reaching feasibility, the MILP solver that finds the optimal solution of a given problem by solving fewer B\&B nodes compared to other MILP solvers is considered faster. In addition to this, if one or more solvers terminate because of time/node/memory limits before finding the optimal solution then the MILP solver that finds the feasible solution with the smaller optimality gap by solving at most the same number of LP relaxation problems as other solvers is 
consider faster. Ties are allowed. The optimality gap is considered zero for the optimal solution regardless of the default optimality tolerance setting of the solver, and is considered infinite for an infeasible solution. The OSR of MILP solver A is defined as:

$$
\begin{gathered}
\text { Number of testcases for which solver } A \text { finds } \\
\text { the optimal or final solution faster than other solvers }
\end{gathered}
$$

In a optimality experiment, if all solvers find the optimal solution of a MILP problem within the specified limits (e.g. time limit) then we can determine the fastest solver(s) among them for this MILP problem (results are comparable). If one or more solvers are not able to find the optimal/feasible solution within the specified limits then we consider such results comparable if and only if:

- At least one solver (say solver A), among the solvers being compared, has found the optimal/feasible solution of this MILP problem and

- For each other solver (say solver B) the number of nodes solved by solver $\mathrm{A} \leq$ the number of nodes solved by solver B and the optimality gap of the solution found by solver $\mathrm{A} \leq$ the optimality gap of the solution found by solver $\mathrm{B}$.

The un-comparable results are not included in the OSR calculations of the solvers. The MILP solver having the largest value of OSR is considered faster for finding the optimal solutions of the MILP problems.

Active Constraint Scheme G, which finds the first feasible solution faster, is the only Active Constraint Scheme that is considered for this experiment. Table 8 lists the Active Constraint Solvers based on Active Constraint Scheme G for finding the optimal solution of the MILP problems. Active Constraint Solver O uses Active Constraint 
Scheme $G$ until the optimal solution is found but Active Constraint Solver $P$ uses Active Constraint Scheme G only until the first feasible solution is found and then it uses the CPLEX default branching variable selection scheme. Both solvers uses DFS node selection until the first feasible solution is found and then use CPLEX default node selection which is close to best bound node selection.

\begin{tabular}{|c|c|c|c|c|}
\hline \multirow{2}{*}{} & \multicolumn{2}{|c|}{$\begin{array}{c}\text { Branching variable selection } \\
\text { scheme }\end{array}$} & \multicolumn{2}{c|}{ Node selection scheme } \\
\cline { 2 - 5 } & $\begin{array}{c}\text { Until First } \\
\text { Feasible } \\
\text { Solution }\end{array}$ & $\begin{array}{c}\text { After First } \\
\text { Feasible } \\
\text { Solution }\end{array}$ & $\begin{array}{c}\text { Until First } \\
\text { Feasible } \\
\text { Solution }\end{array}$ & $\begin{array}{c}\text { After First } \\
\text { Feasible } \\
\text { Solution }\end{array}$ \\
\hline & & & & \\
Active & Active & Active & & CPLEX \\
Solver $\mathbf{O}$ & Scheme G & Scheme G & & Default \\
& & & & DFS \\
Active & Active & CPLEX & DFS & CPLEX \\
Constraint & Constraint & Default & & Default \\
Solver $\mathbb{P}$ & Scheme G & & & \\
\hline
\end{tabular}

Table 8: Designation of the Active Constraint Solvers for finding the optimal solution of the MILP problems

The Active Constraint Solvers of Table 8 are compared individually with the CPLEX 6.5 MILP solver. The CPLEX 6.5 MILP solver is first used to find the optimal solution of the MILP problems in the MIPLIB 3.0 library. The time limit is set to 40,000 seconds and no limit is set on the number of B\&B nodes. For the Active Constraint 
Solvers, the $B \& B$ node limit for solving the MILP problem is set to the number of $B \& B$ nodes solved by the CPLEX 6.5 MLP solver for that problem. In the absence of appropriate data structures, the Active Constraint Scheme takes more time to select the branching variable. For this reason, the time limit for the Active Constraint Solvers is set to 80,000 seconds (double the time limit set for the CPLEX solver). Since the results are compared based on the number of solved B\&B nodes, different time limit on the solvers will not affect the comparison. Also, the OSR calculation performs a fair comparison between the results of different solvers when at least one solver finds a non-optimal solution within the specified limits.

Table 9 shows the OSR of Active Constraint Solvers $\mathrm{O}$ and $\mathrm{P}$ calculated by comparing their results individually with the results of the CPLEX 6.5 MILP solver. The detailed comparison of the results is given in Appendix H.

Note 12 (OSR in Table 9): Since Active Constraint Solver O uses the Active Constraint Scheme throughout the $\mathrm{B} \& \mathrm{~B}$ process to choose the branching variable at each node, it can take a long time to reach the final solution or the node limit. For this reason 18 problems in the MIPLIB 3.0 library (generally the ones for which CPLEX 6.5 must solve a large number of nodes) are not solved using Active Constraint Solver O. These problems are marked "_" in Appendix H. In addition the following problems are marked un-comparable for solver $\mathrm{O}$.

- 10teams, fixnet6, gesa2, mkc, mod011 (5 problems)

The following problems are marked un-comparable for solver P:

- arki001, markshare1, markshare2, pp08a (4 problems) 


\begin{tabular}{|c|c|}
\hline MILP Solver & OSR \\
\hline Active Constraint Solver $\mathbf{O}$ & 0.16 \\
\hline Active Constraint Solver P & 0.41 \\
\hline
\end{tabular}

Table 9: OSR of the Active Constraint Solvers compared individually with the CPLEX 6.5 MILP solver

See Note 12 on page 107

Active Constraint Solver P performs much better than Active Constraint Solver O for finding the optimal/final solution of the MILP problems. The use of Active Constraint Scheme $G$ throughout the $B \& B$ tree is ineffective in finding the optimal solution faster. However if Active Constraint Scheme G is only used until the first feasible solution and the CPLEX 6.5 MILP solver is then allowed to continue from the resulting B\&B tree (as done by Active Constraint Solver P) then the results are comparable to those obtained using the CPLEX 6.5 MILP solver alone. Since Active Constraint Scheme G finds the initial feasible solution faster, which is important in finding the optimal solution faster, one would expect Active Constraint Solver P to perform better than CPLEX 6.5 MILP solver for finding the optimal solution faster. However other factors such as the quality of the feasible solution and the selection criterion used by the branching variable selection and the node selection schemes of CPLEX 6.5 MLP solver impact the performance of the overall algorithm. The quality of the branching variable selection or node selection decisions that are made after the first feasible solution is found might be affected by some 
parameter values (e.g. pseudo-cost) that are calculated at the branch and bound nodes before the first feasible solution is found. Since Active Constraint Solver P uses the active constraint schemes only until the first feasible solution is found, the unavailability of such parameters values to the CPLEX 6.5 after the first feasible solution is found might degrade the quality of its branching variable and node selection decisions to reach the optimal solution. The details of the selection criterion used by the branching variable selection in the CPLEX 6.5 MILP solver are not documented.

The following remarks are made based on the results described in Table 9. The use of the Active Constraint Scheme throughout the B\&B tree is ineffective in finding the optimal solution of the MLP problems faster. Instead, using the Active Constraint Scheme until the first feasible solution is found and then using the branching scheme based on an objective function measure (e.g. pseudo-cost) has a higher probability of finding the optimal solution faster. 


\section{Final Remarks}

\subsection{Conclusions}

The following conclusions are drawn from the observations made in the empirical results of Sections 8.5 and 8.6 :

- The use of the active constraints in the LP relaxation optimal solution for selecting the branching variable at the $B \& B$ node is usually a better approach for finding the first integer feasible solution of a MILP problem.

- Among different branching schemes based on the active constraints, Active Constraint Scheme $G$ is the best method for finding the first feasible solution of a MILP problem.

- The FSR of the solvers (Table 1) using the Active Constraint Schemes that assign weight to a candidate variable relative to its coefficient in an active constraint (Active Constraint Schemes $E$ to $G$ ) are better than those of the solvers using the Active Constraint Schemes that assign equal weight to each candidate variable that occurs in an active constraint (Active Constraint Schemes A to D). The relative size of the coefficient of a variable in a constraint is a good measure of its impact on that constraint.

- If the sole purpose is to find the first feasible solution of a MILP problem faster than CPLEX 6.5 (i.e. ignoring the quality of the solution and the amount of speed-up to reach the solution), Active Constraint Scheme $I$ is the best method assuming that the weight calculation using different Active Constraint Schemes is done quickly. 
- Active Constraint Scheme $\mathrm{J}_{\mathrm{G}}$ assigns weight to a candidate variable in an active constraint based on the relative impact of that constraint on the LP relaxation optimal solution (i.e. dual cost of the constraint). But the FSR, FSF and QSR of the solver using Active Constraint Scheme $J_{G}$ are less than most of the other solvers using the Active Constraint Schemes that give equal weight to each active constraint (Table 1). Hence the dual costs of the active constraints are not important for making the branching variable selection decision. Whether the constraint is active in the LP relaxation optimal solution is all that matters.

- The DFS node selection scheme is the best companion for the Active Constraint Schemes for finding the first feasible solution of the MILP problems faster.

- After the integer feasible solution of the MILP problem is found, it is better to use branching variable selection schemes based on objective function measures (e.g. pseudo-cost) to reach the optimal solution faster.

\subsection{Summary of Contributions}

The following is a summary of contributions to knowledge made by this research:

- Developed a new set of branching schemes that dynamically select the variable based on the active constraints in the LP relaxation optimal solution at the node.

- Demonstrated the superiority of the new branching variable selection schemes for finding the first feasible solution of MILP problems by comparing them with two state-of-the-art commercial solvers (CPLEX 6.5 and OSLMSLV 3.0).

- Developed a set of node selection schemes based on integer infeasibilities at the nodes. 
- Identified the best node selection scheme among the new schemes and the standard DFS scheme for finding the first feasible solution of MILP problems.

- Determined the impact of using the developed branching scheme on algorithm speed in reaching the final solution of the MILP problem by comparing it with the commercial implementation in CPLEX.

\subsection{Future Work}

a) Presolving, Node heuristics and Cut generation influence the speed of the B\&B algorithm in finding the first integer feasible solution of MILP problems. Since these features are available in most modern solvers, their interaction with branching schemes based on the active constraints in finding the first integer feasible solution faster needs to be studied.

b) The Active Constraint Solvers use active constraint information to make the branching variable selection decision. This improves the algorithm speed in finding the first integer feasible solution, but is not effective in finding the optimal solution faster. Most of the existing branching variable selection schemes use objective function information. A branching variable selection scheme can be developed that uses the active constraint and objective function information together. Such a scheme would initially give more importance to the active constraint information for finding a good feasible solution faster which can lead to earlier pruning of the B\&B tree. As the algorithm proceeds, it gradually increases the use of the objective function information and decreases the use of the active constraint information. The impact of 
such a branching scheme on the algorithm speed to find the optimal solution should be studied.

c) For some of the MLP problems, all Active Constraint Schemes perform better than CPLEX 6.5 for finding the first feasible solution faster (e.g. 10teams, swath, misc07, nw04). On the other hand, for some of the MILP problems, CPLEX 6.5 outperforms most of the Active Constraint Schemes (e.g. arki001, air04, flugpl). Also among the Active Constraint Schemes, certain schemes perform better on certain problems than others - for example, Active Constraint Scheme G performs better for the enigma problem, Active Constraint Scheme I for arki001, Active Constraint Scheme B and C for harp2 and Active Constraint Schemes E, F and G for noswot. There are several ways of identifying when to use each of the several branching methods (including CPLEX 6.5) to improve the overall results for finding the integer feasible solution faster:

- The classification problem is to divide a given set of points into classes based on certain attributes such that the number of wrong assignments (compared to actual observation) is minimized. There are algorithms available [Chinneck 1996, 2001] for solving classification problems. Consider for example the experimental results of Table 1 which suggest that, under the given conditions, Active Constraint Solver G can find the first feasible solution of the MLLP problems as fast or faster than CPLEX 6.5 solver in around $70 \%$ of the MLP problems. If one has a choice to select different MILP solvers for finding the first feasible solution of MILP problems then he/she would like to use Active Constraint Solver $\mathrm{G}$ for the subset of the given problems that have a higher chance of finding the first feasible 
solution faster. A classifier algorithm can be used to derive the separating hyperplane inequality based on the available results. This inequality can then be used to decide earlier in the B\&B process whether Active Constraint Solver G should be used to find the integer feasible solution of the MILP problem, or whether the CPLEX standard method should be used.

- In some of the LP relaxations, the set of active constraints is such that the available fractional valued integer variables do not have much impact on the active constraints. In such cases, we can let CPLEX choose the branching variable. Some experimental data were collected while solving flugpl, lseu, swath and enigma using Active Constraint Solver G. CPLEX 6.5 performs better than Active Constraint Solver $\mathrm{G}$ for the flugpl and lseu problems and worse for the swath and enigma problems. The weight of the branching variable selected by Active Constraint Scheme G for flugpl and lseu usually decreases as we go deeper into the $\mathrm{B} \& \mathrm{~B}$ tree. On the other hand, the weight of the branching variable selected by Active Constraint Scheme G for swath and enigma usually remains the same or increases as we go deeper into the $B \& B$ tree. So a branching scheme can be developed that selects the branching variable at a given $\mathrm{B} \& \mathrm{~B}$ node using the Active Constraint Scheme if the weight of the selected branching variable indicates that it has considerable impact on the active constraints. Otherwise the branching variable is selected by CPLEX 6.5 (or other) MILP solver. By using a cutoff condition based on the average weight of the selected branching variable at the first five $B \& B$ nodes, we were able to find the first integer feasible solution of flugpl and 1seu using Active Constraint Scheme G after solving 160 and 18 LP 
relaxations respectively. This is a considerable improvement from the original number of more than 2000 LP relaxations for solving each of these problems using Active Constraint Solver G (without cutoff).

- Since each Active Constraint Scheme performs better on some problems than on others, we can select a particular Active Constraint Scheme at the B\&B node based on the circumstances at that node. The decision-making ingredients of the Active Constraint Schemes, such as the constraints that are active, the variables that are fractional, the variable ordering suggested by different schemes, etc., can be used to determine which Active Constraint Scheme is best suited for particular circumstances. Hence the overall heuristic for selecting the branching variable at each $\mathrm{B} \& \mathrm{~B}$ node would be to check the circumstances and to choose the best variable ordering heuristic. 


\section{References}

1. Arntzen, B. C., G. B. Brown, T. P. Harrison, L. L. Trafton. 1995. Global Supply Chain Management at Digital Equipment Corporation. Interfaces 25, 69-93.

2. Ball, M. O., A. Vakhutinsky. 2001. Fault-Tolerant Virtual Path Layout in ATM Networks. INFORMS Journal On Computing 13, 76-94.

3. Beale, E.M.L. 1968. Mathematical Programming in Practice, Pitmans, London.

4. Beale, E.M.L. 1979. Branch and Bound Methods for Mathematical Programming Systems, Annals of Discrete Mathematics 5, 201-219.

5. Beale, E.M.L., J.A. Tomlin. 1970. Special facilities in a general mathematical programming system for nonconvex problems using ordered sets of variables. Proceedings of the Fifth International Conference on Operational Research (Tavistock publication, London). 447-454.

6. Benichou, M., J. M. Gauthier, P. Girodet, G. Hentges, G. Ribiere, O. Vincent. 1971. Experiments in mixed-integer linear programming. Mathematical Programming 1, 7694.

7. Bixby, R. 1996. Personal communication by Linderoth, J. T. and M. W. P. Savelsbergh [Linderoth and Savelsbergh, 1999].

8. Bixby, R. E., M. Fenelon, Z. Gu, E. Rothberg, R. Wunderling. 2000. MIP: Theory and Practice - Closing the Gap. System Modelling and Optimization: Methods, Theory and Applications. Kluwer Academic Publishers 19-49.

9. Bixby, R. E., S. Ceria, C. M. McZeal, M. W. P. Savelsbergh. 1998. An Updated Mixed Integer Programming Library: MIPLIB 3.0. Optima 58, 12-15. 
10. Chinneck, J. W. 1996. An effective polynomial-time heuristic for the minimumcardinality IIS set-covering problem. Annals of Mathematics and Artificial Intelligence $17,127-144$.

11. Chinneck, J. W. 2001. Fast Heuristics for the Maximum Feasible Subsystem Problem. Informs Journal on Computing 13, 210-223.

12. Cornuejols, G., M. Dawande. 1998. A Class of Hard Small 0-1 Programs. Proceedings of the $6^{\text {th }}$ International IPCO Conference 284-293.

13. Dakin, R. J. 1965. A Tree Search Algorithm for Mixed Integer Programming Problems. Computer Journal 8, 250-255.

14. Dantzig, G. B. 1949. Maximization of a linear function of variables subject to linear inequalities. Proceedings of Linear Programming Conference 20-24.

15. Eckstein, J. 1994. Parallel branch-and-bound algorithms for general mixed integer programming on the CM-5. SIAM Journal on Optimization 4, 794-814.

16. Forest, J. J. H., J. P. H. Hirst, J. A. Tomlin. 1974. Practical Solution of Large Scale Mixed Integer Programming Problems with UMPIRE. Management Science 20, 736773.

17. Garey, M. R., D. S. Johnson. 1979. Computers and Intractability: A Guide to the Theory of NP-Completeness. W. H. Freeman and Company.

18. Gauthier, J. M., G. Ribiere. 1977. Experiments in mixed-integer linear programming. Mathematical Programming 12, 26-47.

19. Gilmore, P., R. Gomory. 1961. A Linear Programming Approach to the Cutting Stock Problem. Operations Research 9, 849-859. 
20. Gomory, R. E. 1958. Outline of an Algorithm for Integer Solutions to Linear Programs. Bulletin of the American Mathematical Society 64, 275-278.

21. Hirst, J. P. H. 1969. Features required in Branch-and-Bound Algorithms for (0-1) Mixed Integer Linear Programming. Unpublished manuscript.

22. IBM Corporation. 1997. MIP Solutions User Guide. Online at http://www6.software.ibm.com/sos/features/oslmslv.htm

23. ILOG Corporation. 1999a. CPLEX 6.5 Advanced Reference Manual.

24. ILOG Corporation. 1999b. CPLEX 6.5 Reference Manual.

25. ILOG Corporation. 1999c. CPLEX 6.5 User's Manual.

26. Johnson E. L., G. L. Nemhauser, M. W. P. Savelsbergh. 2000. Progress in Linear Programming-Based Algorithms for Integer Programming: An Exposition. INFORMS Journal on Computing 12, 2-23.

27. Johnson, E. L. 1989. Modeling and Strong Linear Programs for Mixed Integer Programming. Algorithms and Model Formulations in Mathematical Programming, NATO ASI Series $51,1-41$.

28. Karmarkar, N. 1984. A New Polynomial-Time Algorithm for Linear Programming. Combinatorica 4, 373-395.

29. Land, A. H., A. G. Doig. 1960. An Automatic Method for Solving Discrete Programming Problems. Econometrica 28, 497-520.

30. Linderoth, J. T., M. W. P. Savelsbergh. 1999. A computational study of search strategies for Mixed Integer Programming. INFORMS Journal of Computing 11, 173187. 
31. Mitra, G. 1973. Investigation of Some Branch-and-Bound Strategies for the Solution of Mixed Integer Linear Programs. Mathematical Programming 5, 155-170.

32. Mittelman H. 2001. Benchmark for Optimization Software web-site at hitto://plato.la.asu.edu/bench.html.

33. Padberg M., G. Rinaldi. 1987. Optimization of a 532-city symmetric traveling salesman problem by branch and cut. Oper. Res. Lett. 6, 1-7.

34. Padberg, M. W., G. Rinaldi. 1991. A Branch-and-Cut Algorithm for the Resolution of Large-scale Symmetric Traveling Salesman Problems. SIAM Review 33, 60-100.

35. Savelsbergh, M. W. P. 1994. Preprocessing and Probing Techniques for Mixed Integer Programming Problems. ORSA Journal on Computing 6, 445-454.

36. Sherwani, N. 1993. Algorithms for VLSI Physical Design Automation. Kluwer Academic Publishers.

37. Small, R.E. 1965. Mixed Integer Programming by a Branch and Bound Technique. Proc. of the $3^{\text {rd }}$ IFIP Congress 2, 450-451.

38. Tomlin J. A. 1969. An improved Branch-and-Bound Method for Integer Programming. Operations Research 19, 1070-1075.

39. Vance, P. H., C. Barnhart, E. L. Johnson, G. L. Nemhauser. 1997. Airline crew scheduling: A new formulation and decomposition algorithm. Operations Research $45,188-200$.

40. Winston, W. L. 1995. Introduction to Mathematical Programming. Duxbury Press. Second edition. 


\section{Appendices}

Appendix A describes the MILP problems of the test libraries that were used in the experiments. Appendices $\mathrm{B}$ to $\mathrm{H}$ show tables describing the details of the results obtained using different MILP solvers during feasibility/optimality tests. The rows of these tables represent the MILP problems being solved and the columns represent the MILP solvers. These results tables are used to calculate FSR, FSF, FSF', QSR, QSR' and OSR for the solvers. The numbers appearing in boldface in these tables indicate that the corresponding MILP solver (column) is a winner in the comparison of the results for the particular MILP problem (row). In the following tables, some of the numbers are followed by the ' + ' sign. This sign indicates that the solver was terminated before returning the first feasible solution or the optimal solution (as required) because it reached one of the limits. The optimality gaps are either shown in separate tables or in the brackets in the same table that shows the number of solved nodes. 
Test Model test libraries

MILP problems of MIPLIB 3.0 test library are described below.

\begin{tabular}{|c|c|c|c|c|c|}
\hline Problem & ROWS & COLS & Binary & $\begin{array}{l}\text { General } \\
\text { Integer }\end{array}$ & Real \\
\hline 10teams & 230 & 2025 & 1800 & 0 & 225 \\
\hline air03 & 124 & 10757 & 10757 & 0 & 0 \\
\hline air04 & 823 & 8904 & 8904 & 0 & 0 \\
\hline air05 & 426 & 7195 & 7195 & 0 & 0 \\
\hline arki001 & 1048 & 1388 & 415 & 123 & 850 \\
\hline bell3a & 123 & 133 & 39 & 32 & 62 \\
\hline bell5 & 91 & 104 & 30 & 28 & 46 \\
\hline blend2 & 274 & 353 & 231 & 33 & 89 \\
\hline cap6000 & 2176 & 6000 & 6000 & 0 & 0 \\
\hline dano3mip & 3202 & 13873 & 552 & 0 & 13321 \\
\hline danoint & 664 & 521 & 56 & 0 & 465 \\
\hline dcmulti & 290 & 548 & 75 & 0 & 473 \\
\hline dsbmip & 1182 & 1886 & 160 & 32 & 1694 \\
\hline egout & 98 & 141 & 55 & 0 & 86 \\
\hline enigma & 21 & 100 & 100 & 0 & 0 \\
\hline fast0507 & 507 & 63009 & 63009 & 0 & 0 \\
\hline fiber & 363 & 1298 & 1254 & 0 & 44 \\
\hline fixnet6 & 478 & 878 & 378 & 0 & 500 \\
\hline flugpl & 18 & 18 & 0 & 11 & 7 \\
\hline gen & 780 & 870 & 144 & 6 & 720 \\
\hline gesa 2 & 1392 & 1224 & 240 & 168 & 816 \\
\hline gesa2_o & 1248 & 1224 & 384 & 336 & 504 \\
\hline gesa 3 & 1368 & 1152 & 216 & 168 & 768 \\
\hline gesa3_o & 1224 & 1152 & 336 & 336 & 480 \\
\hline gt2 & 29 & 188 & 24 & 164 & 0 \\
\hline harp 2 & 112 & 2993 & 2993 & 0 & 0 \\
\hline khb05250 & 101 & 1350 & 24 & 0 & 1326 \\
\hline 1152lav & 97 & 1989 & 1989 & 0 & 0 \\
\hline lseu & 28 & 89 & 89 & 0 & 0 \\
\hline markshare 1 & 6 & 62 & 50 & 0 & 12 \\
\hline markshare2 & 7 & 74 & 60 & 0 & 14 \\
\hline $\operatorname{mas} 74$ & 13 & 151 & 150 & 0 & 1 \\
\hline $\operatorname{mas} 76$ & 12 & 151 & 150 & 0 & 1 \\
\hline misc03 & 96 & 160 & 159 & 0 & 1 \\
\hline misc06 & 820 & 1808 & 112 & 0 & 1696 \\
\hline misc07 & 212 & 260 & 259 & 0 & 1 \\
\hline mitre & 2054 & 10724 & 10724 & 0 & 0 \\
\hline $\mathrm{mkc}$ & 3411 & 5325 & 5323 & 0 & 2 \\
\hline $\bmod 008$ & 6 & 319 & 319 & 0 & 0 \\
\hline
\end{tabular}


continue...

\begin{tabular}{|c|c|c|c|c|c|}
\hline Problem & ROWS & COLS & Binary & $\begin{array}{l}\text { General } \\
\text { Integer }\end{array}$ & Real \\
\hline $\bmod 010$ & 146 & 2655 & 2655 & 0 & 0 \\
\hline $\bmod 011$ & 4480 & 10958 & 96 & 0 & 10862 \\
\hline modglob & 291 & 422 & 98 & 0 & 324 \\
\hline noswot & 182 & 128 & 75 & 25 & 28 \\
\hline nw04 & 36 & 87482 & 87482 & 0 & 0 \\
\hline p0033 & 16 & 33 & 33 & 0 & 0 \\
\hline p0201 & 133 & 201 & 201 & 0 & 0 \\
\hline p0282 & 241 & 282 & 282 & 0 & 0 \\
\hline p0548 & 176 & 548 & 548 & 0 & 0 \\
\hline p2756 & 755 & 2756 & 2756 & 0 & 0 \\
\hline pk1 & 45 & 86 & 55 & 0 & 31 \\
\hline pp08a & 136 & 240 & 64 & 0 & 176 \\
\hline pp08aCUTS & 246 & 240 & 64 & 0 & 176 \\
\hline qiu & 1192 & 840 & 48 & 0 & 792 \\
\hline qnet1 & 503 & 1541 & 1288 & 129 & 124 \\
\hline qnet1_o & 456 & 1541 & 1288 & 129 & 124 \\
\hline rentacar & 6803 & 9557 & 55 & 0 & 9502 \\
\hline rgn & 24 & 180 & 100 & 0 & 80 \\
\hline rout & 291 & 556 & 300 & 15 & 241 \\
\hline setlch & 492 & 712 & 240 & 0 & 472 \\
\hline seymour & 4944 & 1372 & 1372 & 0 & 0 \\
\hline stein 27 & 118 & 27 & 27 & 0 & 0 \\
\hline stein 45 & 331 & 45 & 45 & 0 & 0 \\
\hline swath & 884 & 6805 & 6724 & 0 & 81 \\
\hline vpm1 & 234 & 378 & 168 & 0 & 210 \\
\hline $\mathrm{vpm} 2$ & 234 & 378 & 168 & 0 & 210 \\
\hline
\end{tabular}


MILP problems of FCTP test library are described below.

\begin{tabular}{llllll}
\hline Problem & ROWS & COLS & Binary & $\begin{array}{l}\text { General } \\
\text { Integer }\end{array}$ & Real \\
& & & & & \\
& & & & & \\
& & & & & 100 \\
$\operatorname{ran} 10 \times 10 \mathrm{~b}$ & 120 & 200 & 100 & 0 & 120 \\
$\operatorname{ran} 10 \times 12$ & 142 & 240 & 120 & 0 & 100 \\
$\operatorname{ran} 10 \times 10 \mathrm{c}$ & 120 & 200 & 100 & 0 & 169 \\
$\operatorname{ran} 13 \times 13$ & 195 & 338 & 169 & 0 & 260 \\
$\operatorname{ran} 10 \times 26$ & 296 & 520 & 260 & 0 & 256 \\
$\operatorname{ran} 8 \times 32$ & 296 & 512 & 256 & 0 & 256 \\
$\operatorname{ran} 16 \times 16$ & 288 & 512 & 256 & 0 & 252 \\
$\operatorname{ran} 12 \times 21$ & 285 & 504 & 252 & 0 & 144 \\
$\operatorname{ran} 6 \times 43$ & 307 & 516 & 258 & 0 & 252 \\
$\operatorname{ran} 12 \times 12$ & 168 & 288 & 144 & 0 & 289 \\
$\operatorname{ran} 14 \times 18$ & 284 & 504 & 252 & 0 & 256 \\
$\operatorname{ran} 17 \times 17$ & 323 & 578 & 289 & 0 & 100 \\
$\operatorname{ran} 4 \times 64$ & 324 & 512 & 256 & 0 & \\
$\operatorname{ran} 10 \times 10 \mathrm{a}$ & 120 & 200 & 100 & 0 & \\
\hline
\end{tabular}


MILP problems of MIP Benchmarks test library are described below.

\begin{tabular}{llllll}
\hline Problem & ROWS & COLS & Binary & $\begin{array}{l}\text { General } \\
\text { Integer }\end{array}$ & Real \\
\hline & & & & & \\
bc & 1913 & 1751 & 483 & 0 & 1268 \\
haprp & 1048 & 1828 & 0 & 1828 & 0 \\
mas284 & 68 & 151 & 150 & 0 & 1 \\
irp & 39 & 20315 & 20315 & 0 & 0 \\
binkar10_1 & 1026 & 2298 & 170 & 0 & 2128 \\
prod1 & 208 & 250 & 149 & 0 & 101 \\
eilD76 & 75 & 1898 & 1898 & 0 & 0 \\
rip2 & 68 & 451 & 450 & 0 & 1 \\
& & & & & \\
\hline
\end{tabular}


MLLP problems of MP Test Model library are described below.

\begin{tabular}{llllll}
\hline Problem & ROWS & COLS & Binary & $\begin{array}{l}\text { General } \\
\text { Integer }\end{array}$ & Real \\
& & & & & \\
& & & & & \\
swath2 & 884 & 6805 & 2406 & 0 & 4399 \\
bienst1 & 576 & 505 & 28 & 0 & 477 \\
bc1 & 1913 & 1751 & 252 & 0 & 1499 \\
bienst2 & 576 & 505 & 35 & 0 & 470 \\
nug08 & 912 & 1632 & 1632 & 0 & 0 \\
qap10 & 1820 & 4150 & 4150 & 0 & 0 \\
dano3_3 & 3202 & 13873 & 69 & 0 & 13804 \\
dano3_4 & 3202 & 13873 & 92 & 0 & 13781 \\
mkc1 & 3411 & 5325 & 3087 & 0 & 2238 \\
neos1 & 5020 & 2112 & 2112 & 0 & 0 \\
seymour1 & 4944 & 1372 & 451 & 0 & 921 \\
dano3_5 & 3202 & 13873 & 115 & 0 & 13758 \\
neos2 & 1103 & 2101 & 1040 & 0 & 1061 \\
neos3 & 1442 & 2747 & 1360 & 0 & 1387 \\
swath1 & 884 & 6805 & 2306 & 0 & 4499 \\
& & & & & \\
\hline
\end{tabular}


Appendix B: Comparison of the Active Constraint Variable Selection Schemes

(using DFS node selection) vs. CPLEX 6.5 for finding the first feasible solution of

the MULP problems of MIPLIB 3.0 library

The information in the following table is used to calculate FSR, FSF and FSF' of the MILP solvers. For each MILP problem, the number of nodes solved by each solver is shown in the table. The numbers appearing in boldface indicate that the corresponding MILP solver (column) finds the first integer feasible solution faster/equally as fast as the CPLEX 6.5 MILP solver for the given MILP problem (row).

MILP Problem The number of B\&B nodes solved to find the first feasible solution

Name

CPLEX Active Constraint Solver

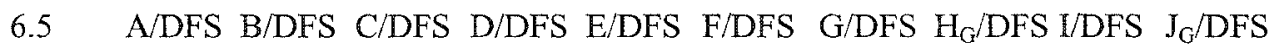

\begin{tabular}{|c|c|c|c|c|c|c|c|c|c|c|c|}
\hline 10teams & 7814 & 234 & 234 & 234 & 661 & 234 & 234 & 661 & 203 & 234 & 1074 \\
\hline air03 & 1 & 6 & 5 & 5 & 5 & 5 & 5 & 5 & 9 & 5 & 5 \\
\hline air04 & 8 & 14 & $1009+$ & $1009+$ & 28 & $1009+$ & $1009+$ & 28 & 114 & $1009+$ & 26 \\
\hline air05 & 35 & 17 & 33 & 33 & 21 & 33 & 33 & 21 & 17 & 33 & 26 \\
\hline arki001 & 296 & $1297+$ & $1297+$ & $1297+$ & $1297+$ & $1297+$ & $1297+$ & $1297+$ & $1297+$ & 189 & $1297+$ \\
\hline bell3a & 16 & 22 & 24 & 23 & 23 & 24 & 22 & 23 & 22 & 23 & 28 \\
\hline bell 5 & 62 & 20 & 19 & 19 & 19 & 19 & 19 & 20 & 20 & 20 & 19 \\
\hline blend 2 & 637 & 45 & $1638+$ & $1638+$ & 98 & $1638+$ & 45 & 45 & 45 & $1638+$ & 79 \\
\hline cap 6000 & 915 & 463 & 463 & 463 & 463 & $1916+$ & $1916+$ & $1916+$ & $1916+$ & $1916+$ & 427 \\
\hline dano3mip & $46+$ & 47 & 47 & 47 & 46 & 47 & 48 & 46 & 46 & 47 & 60 \\
\hline danoint & 769 & 1259 & 779 & $1770+$ & $1770+$ & 1705 & 1702 & 1516 & 660 & 1208 & 400 \\
\hline demulti & 30 & 35 & 51 & 51 & 51 & 44 & 38 & 39 & 48 & 39 & 43 \\
\hline dsbmip & 30 & 18 & 18 & 17 & 14 & 21 & 15 & 15 & 18 & 20 & 32 \\
\hline egout & 14 & 15 & 15 & 15 & 15 & 15 & 15 & 15 & 15 & 15 & 15 \\
\hline enigma & 5975 & $6976+$ & $6976+$ & $6976+$ & $6976+$ & 844 & 844 & 545 & 1075 & 3340 & $6976+$ \\
\hline fast 0507 & 2404 & 37 & 69 & 69 & 43 & 69 & 69 & 43 & 63 & 69 & 65 \\
\hline fiber & 136 & 33 & 37 & 37 & 28 & 37 & 37 & 36 & 18 & 38 & 51 \\
\hline fixnet6 & 168 & 70 & 70 & 70 & 70 & 70 & 70 & 70 & 70 & 70 & 66 \\
\hline flugpl & 65 & 797 & 797 & 801 & $1066+$ & 785 & 785 & $1066+$ & 154 & $1066+$ & $1066+$ \\
\hline gen & 30 & 21 & 21 & 21 & 18 & 20 & 18 & 19 & 21 & 20 & 19 \\
\hline gesa2 & 97 & 63 & 61 & 63 & 66 & 61 & 63 & 65 & 65 & 66 & 66 \\
\hline gesa 2 _o & 110 & 64 & 62 & 64 & 65 & 63 & 64 & 66 & 66 & 66 & 67 \\
\hline gesa3 & 58 & 54 & 59 & 61 & 73 & 53 & 53 & 61 & 53 & 59 & 56 \\
\hline gesa3 o & 59 & 53 & 61 & 74 & 73 & 54 & 54 & 55 & 53 & 53 & 55 \\
\hline
\end{tabular}


continue...

MILP Problem

The number of B\&B nodes solved to find the first feasible solution

Name

CPLEX Active Constraint Solver

6.5 A/DFS B/DFS C/DFS D/DFS E/DFS F/DFS G/DFS $\mathrm{H}_{\mathrm{G}} / \mathrm{DFS}$ I/DFS $\mathrm{J}_{\mathrm{G}} / \mathrm{DFS}$

\begin{tabular}{|c|c|c|c|c|c|c|c|c|c|c|c|}
\hline $\mathrm{gt} 2$ & 84 & 20 & 22 & 22 & 18 & 21 & 21 & 21 & 21 & 23 & 15 \\
\hline harp2 & 392 & $1393+$ & 118 & $\mathbb{1 1 8}$ & $1393+$ & $1393+$ & $1393+$ & $1393+$ & $1393+$ & 139 & $1393+$ \\
\hline khb05250 & 22 & 18 & 18 & 18 & 18 & 18 & 18 & 18 & 18 & 18 & 18 \\
\hline 1152 lav & 17 & 28 & 7 & 7 & 33 & 7 & 7 & 29 & 7 & 7 & 15 \\
\hline 1seu & 146 & 754 & 15 & 15 & 434 & 15 & 15 & $1147+$ & $1147+$ & 36 & 16 \\
\hline markshare1 & 27 & 57 & 57 & 57 & 57 & 56 & 56 & 23 & 62 & 26 & 26 \\
\hline markshare2 & 42 & 70 & 70 & 70 & 70 & 71 & 71 & 66 & 28 & 73 & 71 \\
\hline $\operatorname{mas} 74$ & 82 & 19 & 21 & 21 & 21 & 21 & 21 & 21 & 21 & 21 & 21 \\
\hline $\operatorname{mas} 76$ & 81 & 16 & 16 & 16 & 16 & 16 & 16 & 16 & 16 & 16 & 16 \\
\hline $\operatorname{misc} 03$ & 65 & 4 & 4 & 4 & 5 & 6 & 6 & 5 & 5 & 6 & 6 \\
\hline $\operatorname{misc} 06$ & 20 & 20 & 20 & 20 & 20 & 20 & 20 & 20 & 20 & 20 & 20 \\
\hline $\operatorname{misc} 07$ & 282 & 6 & 8 & 8 & 8 & 9 & 9 & 7 & 7 & 8 & 6 \\
\hline mitre & 279 & 110 & 88 & 88 & 116 & 82 & 82 & 72 & 58 & 84 & 75 \\
\hline mkc & 144 & 142 & 169 & 169 & 194 & 161 & 161 & 213 & 360 & 176 & $1145+$ \\
\hline $\bmod 008$ & 202 & 7 & 6 & 6 & 6 & 7 & 7 & 7 & 7 & 7 & 6 \\
\hline $\bmod 010$ & 24 & 25 & 32 & 32 & 25 & 34 & 34 & 23 & 34 & 34 & 6 \\
\hline $\bmod 011$ & 48 & 17 & 17 & 17 & 17 & 15 & 17 & 17 & 17 & 17 & 17 \\
\hline modglob & 64 & 32 & 32 & 32 & 32 & 32 & 32 & 32 & 32 & 32 & 31 \\
\hline noswot & 33 & $1034+$ & $1034+$ & $1034+$ & $1034+$ & 53 & 53 & 55 & 61 & $1034+$ & $1034+$ \\
\hline nw04 & 468 & 3 & 4 & 4 & 3 & 4 & 4 & 3 & 3 & 4 & 3 \\
\hline p0033 & 17 & 82 & 22 & 22 & 22 & 26 & 26 & 176 & 55 & 22 & 974 \\
\hline p0201 & 67 & 13 & 11 & 11 & 11 & 11 & 11 & 13 & 13 & 11 & 9 \\
\hline p0282 & 22 & 47 & 56 & 56 & 49 & 52 & 52 & 47 & 54 & 46 & 57 \\
\hline p0548 & 129 & 89 & 79 & 79 & 93 & 76 & 76 & 87 & 83 & 87 & 87 \\
\hline $\mathrm{p} 2756$ & 407 & 30 & 29 & 29 & 29 & 31 & 31 & 27 & 33 & 25 & 149 \\
\hline pk1 & 42 & 26 & 26 & 26 & 26 & 24 & 24 & 24 & 24 & 25 & 34 \\
\hline pp08a & 54 & 48 & 49 & 49 & 49 & 49 & 48 & 48 & 48 & 49 & 49 \\
\hline pp08aCUTS & 52 & 30 & 36 & 36 & 36 & 32 & 30 & 30 & 33 & 35 & 31 \\
\hline qiu & 35 & 22 & 35 & 35 & 35 & 22 & 22 & 22 & 24 & 22 & 32 \\
\hline qnet1 & 22 & 133 & 37 & 37 & 68 & 120 & 120 & 54 & 126 & 17 & 27 \\
\hline qnet 1 o & 31 & 142 & 101 & 101 & 97 & 127 & 127 & 71 & 138 & 154 & 44 \\
\hline rentacar & 14 & 9 & 15 & 15 & 15 & 10 & 9 & 9 & 9 & 9 & 14 \\
\hline rgn & 58 & 5 & 5 & 5 & 5 & 5 & 5 & 5 & 5 & 5 & 5 \\
\hline rout & 93 & $1094+$ & 44 & 41 & 50 & 46 & 44 & 63 & 40 & 71 & $1094+$ \\
\hline set1ch & 152 & 93 & 142 & 142 & 142 & 100 & 93 & 93 & 93 & 96 & 140 \\
\hline seymour & 131 & 98 & 110 & 110 & 111 & 110 & 110 & 111 & 214 & 110 & 252 \\
\hline stein 27 & 18 & 16 & 16 & 16 & 16 & 16 & 16 & 16 & 20 & 16 & 19 \\
\hline stein 45 & 30 & 28 & 28 & 28 & 29 & 28 & 28 & 29 & 30 & 27 & 34 \\
\hline swath & 6206 & 32 & 34 & 34 & 25 & 26 & 26 & 27 & 31 & 27 & 27 \\
\hline vpm1 & 38 & 22 & 22 & 22 & 22 & 22 & 22 & 22 & 22 & 23 & 22 \\
\hline vpm2 & 435 & 29 & 33 & 33 & 33 & 29 & 29 & 29 & 32 & 31 & 26 \\
\hline
\end{tabular}

Total \# of results faster/

equal as fast as the 
CPLEX 6.5 solver

Total \# of results comparable to that of the CPLEX 6.5 solver 65

65

65

$64 \quad 65$

65

65

64 
The information in the following table is used to calculate the QSR and QSR' of the MLP solvers. The numbers appearing in boldface indicate that the corresponding MILP solver (column) finds the first integer feasible solution of better/same quality and at least not slower than the CPLEX 6.5 MLP solver for the given MLP problem (row). A "--." sign indicates that no integer feasible solution for that MILP problem is found by the solver within the specified limits.

MILP Problem $\quad \%$ optimality gap at the first integer feasible solution

Name

CPLEX Active Constraint Solver

6.5 A/DFS B/DFS C/DFS D/DFS E/DFS F/DFS G/DFS $\mathrm{H}_{\mathrm{G}} / \mathrm{DFS}$ I/DFS $\mathrm{J}_{\mathrm{G}} / \mathrm{DFS}$

\begin{tabular}{|c|c|c|c|c|c|c|c|c|c|c|c|}
\hline 10teams & 1.29 & 7.00 & 7.00 & 7.00 & 0.76 & 7.00 & 7.00 & 0.76 & 6.43 & 7.00 & 2.65 \\
\hline air03 & 0.00 & 12.57 & 1.19 & 1.19 & 8.15 & 1.19 & 1.19 & 8.15 & 9.04 & 1.19 & 2.07 \\
\hline air04 & 1.46 & 5.11 & -- & -- & 6.99 & -- & -- & 6.99 & 17.20 & -- & 6.54 \\
\hline air05 & 5.58 & 21.36 & 22.53 & 22.53 & 3.71 & 22.53 & 22.53 & 3.71 & 2.42 & 22.53 & 18.08 \\
\hline arki001 & 0.19 & -- & -- & -- & -- & -- & -- & -- & -- & 0.41 & -- \\
\hline bell3a & 0.78 & 48.73 & 50.42 & 48.73 & 48.73 & 50.42 & 46.97 & 48.73 & 46.97 & 49.01 & 50.75 \\
\hline bell5 & 3.01 & 3.95 & 3.34 & 3.34 & 3.34 & 3.34 & 3.34 & 3.95 & 3.95 & 3.34 & 3.95 \\
\hline blend2 & 25.93 & 21.54 & -- & -- & 80.87 & -- & 20.37 & 21.54 & 21.54 & - & 85.72 \\
\hline cap 6000 & 0.04 & 7.88 & 7.88 & 7.88 & 7.88 & -- & -- & -- & -- & - & 2.87 \\
\hline dano3mip & -- & 27.92 & 33.90 & 31.90 & 35.60 & 33.27 & 36.47 & 26.67 & 40.60 & 30.59 & 35.83 \\
\hline danoint & 18.52 & 29.62 & 23.14 & -- & -- & 29.62 & 29.62 & 29.62 & 26.31 & 29.62 & 19.70 \\
\hline dcmulti & 1.80 & 31.19 & 33.22 & 33.22 & 33.22 & 27.84 & 23.21 & 23.21 & 34.19 & 28.36 & 27.89 \\
\hline dsbmip & 8.35 & 5.67 & 1166.3 & 20.00 & 0.00 & 1458.49 & 95.67 & 0.00 & 5.67 & 0.10 & 3.02 \\
\hline egout & 38.23 & 41.44 & 41.44 & 41.44 & 41.44 & 41.44 & 41.44 & 41.44 & 41.44 & 42.19 & 43.39 \\
\hline enigma & 0.00 & -- & - & -- & -- & 0.00 & 0.00 & 0.00 & 0.00 & 0.00 & -- \\
\hline fast 0507 & 4.89 & 4.89 & 4.89 & 4.89 & 1.63 & 4.89 & 4.89 & 1.63 & 6.95 & 4.89 & 5.41 \\
\hline fiber & 69.64 & 93.95 & 94.90 & 94.90 & 95.30 & 94.90 & 94.90 & 95.62 & 92.19 & 94.91 & 95.50 \\
\hline fixnet6 & 83.40 & 91.46 & 91.46 & 91.46 & 91.46 & 91.46 & 91.46 & 91.46 & 91.46 & 91.46 & 91.81 \\
\hline flugpl & 2.47 & 5.85 & 5.85 & 5.85 & -- & 5.85 & 5.85 & -- & 5.85 & -- & -- \\
\hline gen & 0.25 & 0.10 & 0.10 & 0.10 & 0.15 & 0.13 & 0.10 & 0.10 & 0.10 & 0.10 & 0.08 \\
\hline gesa2 & 1.98 & 1.27 & 1.34 & 1.34 & 1.35 & 1.27 & 1.34 & 1.27 & 1.28 & 1.34 & 1.34 \\
\hline gesa2_o & 2.37 & 1.34 & 1.34 & 1.27 & 1.35 & 1.34 & 1.34 & 1.34 & 1.28 & 1.34 & 1.34 \\
\hline gesa3 & 1.30 & 1.27 & 1.62 & 1.77 & 2.05 & 1.27 & 1.27 & 1.41 & 0.65 & 1.41 & 0.70 \\
\hline gesa3_o & 1.31 & 1.27 & 1.62 & 2.07 & 2.08 & 1.27 & 1.27 & 0.65 & 0.65 & 0.70 & 0.65 \\
\hline$g t 2$ & 86.51 & 85.41 & 63.37 & 63.37 & 67.39 & 65.65 & 65.65 & 83.44 & 83.44 & 68.29 & 55.08 \\
\hline harp2 & 5.95 & -- & 28.12 & 28.12 & -- & -- & -- & -- & -- & 24.35 & - \\
\hline khb05250 & 12.66 & 21.09 & 21.09 & 21.09 & 21.09 & 21.09 & 21.09 & 21.09 & 21.09 & 21.09 & 21.09 \\
\hline $1152 \mathrm{lav}$ & 4.91 & 5.19 & 2.46 & 2.46 & 5.28 & 2.46 & 2.46 & 5.11 & 2.40 & 2.46 & 4.91 \\
\hline lseu & 33.99 & 50.43 & 40.51 & 40.51 & 37.34 & 40.51 & 40.51 & -- & -- & 49.81 & 50.90 \\
\hline markshare 1 & 100.00 & 100.00 & 100.00 & 100.00 & 100.00 & 100.00 & 100.00 & 100.00 & 100.00 & 100.00 & 100.00 \\
\hline markshare2 & 100.00 & 100.00 & 100.00 & 100.00 & 100.00 & 100.00 & 100.00 & 100.00 & 100.00 & 100.00 & 100.00 \\
\hline
\end{tabular}


continue...

MILP Problem

Name

CPLEX Active Constraint Solver

6.5 A/DFS B/DFS C/DFS D/DFS E/DFS F/DFS G/DFS $H_{G} /$ DFS I/DFS $J_{G} /$ DFS

\begin{tabular}{|c|c|c|c|c|c|c|c|c|c|c|c|}
\hline $\operatorname{mas} 74$ & 38.89 & 46.45 & 39.59 & 39.59 & 33.96 & 52.87 & 52.87 & 40.40 & 33.59 & 34.83 & 44.63 \\
\hline $\operatorname{mas} 76$ & 13.10 & 15.74 & 14.76 & 14.76 & 10.47 & 14.17 & 14.17 & 12.64 & 11.83 & 8.38 & 15.06 \\
\hline $\operatorname{misc} 03$ & 58.61 & 43.15 & 48.10 & 48.10 & 48.10 & 61.80 & 61.80 & 48.10 & 47.53 & 53.41 & 55.94 \\
\hline misc06 & 0.06 & 0.19 & 0.19 & 0.19 & 0.19 & 0.19 & 0.19 & 0.19 & 0.19 & 0.19 & 0.06 \\
\hline $\operatorname{misc} 07$ & 62.86 & 57.12 & 60.14 & 60.14 & 59.69 & 60.14 & 60.14 & 60.14 & 59.51 & 60.14 & 50.61 \\
\hline mitre & 0.00 & 0.62 & 0.53 & 0.53 & 0.95 & 1.70 & 1.70 & 1.71 & 1.25 & 1.06 & 1.57 \\
\hline $\mathrm{mkc}$ & 67.21 & 85.88 & 138.80 & 138.80 & 60.44 & 102.90 & 102.90 & 61.54 & 67.91 & 99.59 & -- \\
\hline $\bmod 008$ & 5.24 & 21.58 & 5.54 & 5.54 & 5.54 & 22.00 & 22.00 & 20.73 & 20.73 & 22.42 & 5.23 \\
\hline $\bmod 010$ & 0.39 & 0.67 & 12.13 & 12.13 & 2.02 & 9.04 & 9.04 & 1.48 & 1.85 & 9.04 & 0.32 \\
\hline $\bmod 011$ & 192.95 & 44.80 & 44.80 & 44.80 & 44.80 & 36.80 & 44.80 & 44.80 & 44.80 & 44.80 & 44.80 \\
\hline modglob & 30.10 & 1.78 & 1.78 & 1.78 & 1.78 & 1.78 & 1.78 & 1.78 & 1.78 & 1.78 & 1.60 \\
\hline noswot & 26.47 & -- & -- & -- & -- & 26.47 & 26.47 & 22.86 & 26.47 & - & -- \\
\hline nw04 & 7.32 & 5.75 & 3.92 & 3.92 & 5.75 & 3.92 & 3.92 & 5.75 & 10.46 & 3.92 & 10.46 \\
\hline p0033 & 28.17 & 20.94 & 22.30 & 22.30 & 18.40 & 22.30 & 22.30 & 20.94 & 18.40 & 22.30 & 24.60 \\
\hline p0201 & 14.65 & 10.89 & 12.92 & 12.92 & 12.92 & 12.92 & 12.92 & 10.89 & 10.89 & 12.92 & 11.58 \\
\hline p0282 & 48.75 & 54.03 & 53.41 & 53.41 & 54.59 & 53.36 & 53.36 & 54.03 & 53.63 & 43.82 & 53.09 \\
\hline p0548 & 93.14 & 97.65 & 97.58 & 97.58 & 97.65 & 97.61 & 97.61 & 97.65 & 97.66 & 97.65 & 97.66 \\
\hline $\mathrm{p} 2756$ & 86.28 & 92.99 & 92.53 & 92.53 & 92.58 & 92.71 & 92.71 & 89.71 & 92.95 & 92.56 & 22.71 \\
\hline $\mathrm{pk} 1$ & 100.00 & 100.00 & 100.00 & 100.00 & 100.00 & 100.00 & 100.00 & 100.00 & 100.00 & 100.00 & 100.00 \\
\hline pp08a & 72.60 & 78.38 & 79.38 & 79.38 & 79.38 & 78.99 & 78.38 & 78.38 & 78.38 & 79.30 & 78.99 \\
\hline pp08aCUTS & 38.35 & 41.63 & 49.53 & 49.53 & 49.53 & 40.04 & 41.63 & 41.63 & 45.25 & 43.67 & 43.96 \\
\hline qiu & 333.02 & 376.88 & 159.07 & 159.07 & 159.07 & 771.55 & 376.88 & 376.88 & 313.30 & 347.69 & 169.06 \\
\hline qnet1 & 14.44 & 94.34 & 77.07 & 77.07 & 88.19 & 93.50 & 93.50 & 83.49 & 92.77 & 62.45 & 62.34 \\
\hline qnet1_o & 35.20 & 95.30 & 93.47 & 93.47 & 91.26 & 95.35 & 95.35 & 91.88 & 94.71 & 95.19 & 86.07 \\
\hline rentacar & 4.71 & 52.91 & 91.56 & 91.56 & 91.56 & 90.70 & 52.91 & 52.91 & 52.91 & 52.91 & 4.71 \\
\hline rgn & 40.63 & 40.63 & 40.63 & 40.63 & 67.89 & 40.63 & 40.63 & 40.63 & 40.63 & 40.63 & 40.63 \\
\hline rout & 31.05 & -- & 30.15 & 30.15 & 21.40 & 30.24 & 30.24 & 27.61 & 20.29 & 35.70 & -- \\
\hline setlch & 62.41 & 54.47 & 67.35 & 67.35 & 67.35 & 56.41 & 54.47 & 54.47 & 54.47 & 55.50 & 66.58 \\
\hline seymour & 8.16 & 8.01 & 7.37 & 7.37 & 7.37 & 7.37 & 7.37 & 7.37 & 16.21 & 7.37 & 13.15 \\
\hline stein 27 & 31.58 & 31.58 & 31.58 & 31.58 & 31.58 & 31.58 & 31.58 & 31.58 & 35.00 & 31.58 & 31.58 \\
\hline stein 45 & 33.33 & 33.33 & 29.03 & 29.03 & 31.25 & 29.03 & 29.03 & 31.25 & 33.33 & 29.03 & 35.29 \\
\hline swath & 58.95 & 50.37 & 47.05 & 47.05 & 43.30 & 53.73 & 53.73 & 53.54 & 53.57 & 53.54 & 48.07 \\
\hline vpm 1 & 25.40 & 32.97 & 32.97 & 32.97 & 32.97 & 32.97 & 32.97 & 32.97 & 32.97 & 35.76 & 32.97 \\
\hline vpm2 & 42.36 & 42.73 & 48.60 & 48.60 & 49.88 & 45.82 & 42.73 & 42.73 & 45.82 & 47.93 & 38.32 \\
\hline \multicolumn{2}{|c|}{$\begin{array}{l}\text { Total \# of better/equal } \\
\text { optimality gap for the } \\
\text { results that are faster/ } \\
\text { equal to CPLEX } 6.5\end{array}$} & $17 / 6$ & $16 / 5$ & $17 / 5$ & $21 / 3$ & $17 / 6$ & $19 / 5$ & $22 / 6$ & $21 / 6$ & $19 / 7$ & $16 / 7$ \\
\hline \multicolumn{2}{|c|}{$\begin{array}{l}\text { Total \# of faster/equal } \\
\text { results compared to } \\
\text { CPLEX } 6.5\end{array}$} & $42 / 2$ & $40 / 3$ & $40 / 3$ & $39 / 2$ & $43 / 2$ & $44 / 1$ & $44 / 1$ & $43 / 2$ & $46 / 2$ & $41 / 2$ \\
\hline
\end{tabular}


Appendix C: Comparison of the Node Selection Schemes (using Active Constraint

Scheme G) vs. CPLEX 6.5 for finding the first feasible solution of the MILP

problems of MIPLIB 3.0 library

The information in the following table is used to calculate FSR, FSF and FSF' of the MILP solvers. For each MILP problem, the number of nodes solved by each solver is shown in the table. The numbers appearing in boldface indicate that the corresponding MILP solver (column) finds the first integer feasible solution faster/equal to CPLEX 6.5 MILP solver for the given MLP problem (row). Please note that a "-..." sign in these tables indicates that the solution for the corresponding MILP problem is not available from that solver. This can happen if the solver fails before reaching the solution of that problem.

\begin{tabular}{|c|c|c|c|c|c|c|}
\hline \multirow{3}{*}{$\begin{array}{l}\text { MILP Problem } \\
\text { Name }\end{array}$} & \multicolumn{6}{|c|}{ Number of B\&B nodes solved to find first feasible solution } \\
\hline & \multirow{2}{*}{$\begin{array}{l}\text { CPLEX } 6.5 \\
\text { MULP Solver }\end{array}$} & \multicolumn{3}{|c|}{ Active Constraint Solver } & \multirow[b]{2}{*}{$\mathrm{M} / \mathrm{DFS}$} & \multirow[b]{2}{*}{ N/DFS } \\
\hline & & G/DFS & K/DFS & L/DFS & & \\
\hline 10teams & 7814 & 661 & 373 & 29 & 537 & 645 \\
\hline air03 & 1 & 5 & 5 & 7 & 5 & 5 \\
\hline air04 & 8 & 28 & 51 & 64 & 47 & 47 \\
\hline air05 & 35 & 21 & 22 & 23 & 27 & 27 \\
\hline arki001 & 296 & $1297+$ & 378 & $1297+$ & $1297+$ & $1297+$ \\
\hline bell3a & 16 & 23 & 11 & 11 & 24 & 24 \\
\hline bell5 & 62 & 20 & 155 & 18 & $1063+$ & $1063+$ \\
\hline blend2 & 637 & 45 & 326 & $1638+$ & 543 & 204 \\
\hline cap6000 & 915 & $1916+$ & $1916+$ & $1916+$ & 1196 & $1916+$ \\
\hline dano3mip & $46+$ & 46 & $46+$ & $58+$ & $50+$ & 78 \\
\hline danoint & 769 & 1516 & 1106 & 94 & 1725 & 1106 \\
\hline dcmulti & 30 & 39 & 36 & 48 & 37 & 36 \\
\hline dsbmip & 30 & 15 & 22 & 22 & 18 & 28 \\
\hline egout & 14 & 15 & 15 & 16 & 15 & 15 \\
\hline enigma & 5975 & 545 & 694 & 145 & -- & --- \\
\hline fast0507 & 2404 & 43 & 120 & 59 & 90 & 109 \\
\hline fiber & 136 & 36 & 70 & 29 & $1137+$ & $1137+$ \\
\hline fixnet6 & 168 & 70 & 54 & 70 & 54 & 54 \\
\hline flugpl & 65 & $1066+$ & 237 & $1066+$ & 201 & $1066+$ \\
\hline
\end{tabular}


continue...

\begin{tabular}{|c|c|c|c|c|c|c|}
\hline \multirow{3}{*}{$\begin{array}{l}\text { MILP Problem } \\
\text { Name }\end{array}$} & \multicolumn{5}{|c|}{ Number of $B \& B$ nodes solved to find first feasible solution } & \multirow[b]{3}{*}{ N/DFS } \\
\hline & \multirow{2}{*}{$\begin{array}{l}\text { CPLEX } 6.5 \\
\text { MILP Solver }\end{array}$} & \multicolumn{3}{|c|}{ Active Constraint Solver } & \multirow[b]{2}{*}{ M/DFS } & \\
\hline & & G/DFS & K/DFS & $\mathrm{L} / \mathrm{DFS}$ & & \\
\hline gen & 30 & 19 & 20 & 21 & 21 & 19 \\
\hline gesa2 & 97 & 65 & 71 & 105 & 78 & 74 \\
\hline gesa2_o & 110 & 66 & 66 & 107 & 68 & 71 \\
\hline gesa3 & 58 & 61 & 72 & 65 & 78 & 70 \\
\hline gesa3_o & 59 & 55 & 90 & 58 & 79 & 77 \\
\hline $\mathrm{gt} 2$ & 84 & 21 & 21 & 670 & 24 & 21 \\
\hline harp2 & 392 & $1393+$ & $1393+$ & $1393+$ & $\cdots$ & --- \\
\hline khb05250 & 22 & 18 & 18 & 18 & 18 & 18 \\
\hline 1152lav & 17 & 29 & 92 & 72 & 33 & 33 \\
\hline lseu & 146 & $1147+$ & 20 & 66 & 22 & 30 \\
\hline markshare1 & 27 & 23 & 27 & 99 & 23 & 23 \\
\hline markshare2 & 42 & 66 & 69 & 248 & 66 & 66 \\
\hline $\operatorname{mas} 74$ & 82 & 21 & 24 & 65 & 21 & 21 \\
\hline $\operatorname{mas} 76$ & 81 & 16 & 18 & 77 & 16 & 16 \\
\hline $\operatorname{misc} 03$ & 65 & 5 & 8 & 21 & 5 & 5 \\
\hline $\operatorname{misc} 06$ & 20 & 20 & 22 & 23 & 21 & 22 \\
\hline misc07 & 282 & 7 & 10 & 15 & 7 & 7 \\
\hline mitre & 279 & 72 & 72 & 204 & 151 & 120 \\
\hline $\mathrm{mkc}$ & 144 & 213 & 301 & 707 & 325 & 438 \\
\hline $\bmod 008$ & 202 & 7 & 8 & 14 & 13 & 8 \\
\hline $\bmod 010$ & 24 & 23 & 41 & 50 & 41 & 41 \\
\hline $\bmod 011$ & 48 & 17 & 17 & 17 & 17 & 17 \\
\hline modglob & 64 & 32 & 32 & 32 & 33 & 32 \\
\hline noswot & 33 & 55 & 290 & 101 & 126 & 435 \\
\hline nw04 & 468 & 3 & 3 & 8 & 8 & 8 \\
\hline p0033 & 17 & 176 & 414 & 33 & 63 & 271 \\
\hline p0201 & 67 & 13 & 13 & 14 & 13 & 13 \\
\hline p0282 & 22 & 47 & 42 & 44 & 34 & 197 \\
\hline p0548 & 129 & 87 & 167 & $1130+$ & $1130+$ & $1130+$ \\
\hline p2756 & 407 & 27 & 40 & 90 & 153 & 43 \\
\hline pk1 & 42 & 24 & 32 & 100 & $1043+$ & 24 \\
\hline pp08a & 54 & 48 & 48 & 50 & 51 & 48 \\
\hline pp08aCUTS & 52 & 30 & 32 & 33 & 38 & 30 \\
\hline qiu & 35 & 22 & 26 & 22 & 26 & 26 \\
\hline qnet1 & 22 & 54 & 29 & 18 & 15 & 13 \\
\hline qnet1_o & 31 & 71 & 925 & 76 & 31 & 47 \\
\hline rentacar & 14 & 9 & 9 & 36 & 9 & 9 \\
\hline $\operatorname{rgn}$ & 58 & 5 & 5 & 5 & 5 & 5 \\
\hline rout & 93 & 63 & 253 & 187 & 115 & 235 \\
\hline setlch & 152 & 93 & 99 & 153 & 107 & 99 \\
\hline seymour & 131 & 111 & 163 & 148 & 145 & 115 \\
\hline stein27 & 18 & 16 & 16 & 19 & 16 & 16 \\
\hline stein 45 & 30 & 29 & 29 & 33 & 29 & 35 \\
\hline
\end{tabular}


continue...

MILP Problem Number of B\&B nodes solved to find first feasible solution

Name

CPLEX 6.5 Active Constraint Solver

$\begin{array}{lllll}\text { MILP Solver G/DFS } & \text { K/DFS } & \text { L/DFS } & \text { M/DFS } & \text { N/DFS }\end{array}$

$\begin{array}{llllllll}\text { swath } & 6206 & 27 & 32 & 154 & 44 & 43\end{array}$

vpm1 38

vpm2 435

2230

29

$30 \quad 27$

$29 \quad 35$

46

43

35

44

30

40

Total \# of results faster/equal to results of CPLEX 6.5 solver

$44 / 1$

$38 / 1$

$34 / 0$

$35 / 1$

$37 / 0$

Total \# of results comparable to that of CPLEX 6.5 solver 65

64

64

62

62 
The information in the following table is used to calculate the QSR and QSR' of the MILP solvers. The numbers appearing in boldface indicate that the corresponding MILP solver (column) finds the first integer feasible solution of better/same quality and at least not slower than the CPLEX 6.5 MLP solver for the given MILP problem (row). The "--" sign below indicates that no integer feasible solution for that MILP problem is found by the solver within the specified limits.

MILP Problem

Name

\begin{tabular}{|c|c|c|c|c|c|c|}
\hline \multirow{2}{*}{. } & \multirow{2}{*}{$\begin{array}{l}\text { CPLEX } \\
6.5\end{array}$} & \multicolumn{3}{|c|}{ Active Constraint Solver } & \multirow[b]{2}{*}{ M/DFS } & \multirow[b]{2}{*}{$\mathrm{N} / \mathrm{DFS}$} \\
\hline & & G/DFS & $\mathrm{K} / \mathrm{DFS}$ & L/DFS & & \\
\hline 10teams & 1.29 & 0.76 & 0.76 & 0.76 & 0.76 & 0.76 \\
\hline air03 & 0.00 & 8.15 & 8.15 & 8.15 & 8.15 & 8.15 \\
\hline air04 & 1.46 & 6.99 & 2.31 & 5.24 & 5.53 & 5.53 \\
\hline air05 & 5.58 & 3.71 & 3.71 & 3.20 & 3.75 & 3.75 \\
\hline arki001 & 0.19 & - & 0.10 & -- & - & - \\
\hline bell3a & 0.78 & 48.73 & 3.02 & 3.02 & 46.95 & 46.95 \\
\hline bell5 & 3.01 & 3.95 & 4.99 & 2.74 & -- & -- \\
\hline blend 2 & 25.93 & 21.54 & 67.56 & -- & 34.14 & 42.82 \\
\hline cap6000 & 0.04 & -- & - & -- & 0.01 & - \\
\hline dano3mip & -- & 26.67 & -- & - & - & 31.41 \\
\hline danoint & 18.52 & 29.62 & 29.62 & 14.20 & 28.82 & 29.62 \\
\hline demulti & 1.80 & 23.21 & 7.15 & 5.33 & 10.52 & 7.15 \\
\hline dsbmip & 8.35 & 0.00 & 1499.84 & 1.30 & 1.30 & 5.67 \\
\hline egout & 38.23 & 41.44 & 41.44 & 42.20 & 40.54 & 41.44 \\
\hline enigma & 0.00 & 0.00 & 0.00 & 0.00 & -- & -- \\
\hline fast0507 & 4.89 & 1.63 & 2.74 & 2.19 & 2.74 & 3.29 \\
\hline fiber & 69.64 & 95.62 & 92.79 & 92.66 & - & - \\
\hline fixnet 6 & 83.40 & 91.46 & 89.52 & 91.46 & 89.52 & 89.52 \\
\hline flugpl & 2.47 & - & 5.92 & - & 5.95 & -- \\
\hline gen & 0.25 & 0.10 & 0.15 & 0.09 & 0.15 & 0.15 \\
\hline gesa2 & 1.98 & 1.27 & 1.66 & 2.63 & 1.60 & 1.39 \\
\hline gesa2_o & 2.37 & 1.34 & 1.42 & 1.63 & 1.44 & 1.41 \\
\hline gesa3 & 1.30 & 1.41 & 1.00 & 0.88 & 2.00 & 1.65 \\
\hline gesa3_o & 1.31 & 0.65 & 2.38 & 0.94 & 1.86 & 2.16 \\
\hline $\mathrm{gt} 2$ & 86.51 & 83.44 & 83.68 & 92.23 & 83.68 & 83.68 \\
\hline harp2 & 5.95 & -- & -- & -- & -- & -- \\
\hline khb05250 & 12.66 & 21.09 & 21.09 & 21.09 & 21.09 & 21.09 \\
\hline $11521 a v$ & 4.91 & 5.11 & 5.34 & 2.61 & 5.11 & 5.11 \\
\hline Iseu & 33.99 & -- & 38.90 & 51.22 & 38.90 & 38.90 \\
\hline markshare 1 & 100.00 & 100.00 & 100.00 & 100.00 & 100.00 & 100.00 \\
\hline markshare2 & 100.00 & 100.00 & 100.00 & 100.00 & 100.00 & 100.00 \\
\hline
\end{tabular}

$\%$ optimality gap at the first integer feasible solution 
continue...

MILP Problem $\quad \%$ optimality gap at the first integer feasible solution Name

CPLEX
6.5

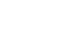

\begin{tabular}{|c|c|c|c|c|c|c|}
\hline $\operatorname{mas} 74$ & 38.89 & 40.40 & 40.07 & 32.41 & 40.40 & 40.40 \\
\hline $\operatorname{mas} 76$ & 13.10 & 12.64 & 8.49 & 20.85 & 12.64 & 12.64 \\
\hline $\operatorname{misc} 03$ & 58.61 & 48.10 & 47.53 & 48.93 & 48.10 & 48.10 \\
\hline $\operatorname{misc} 06$ & 0.06 & 0.19 & 0.33 & 0.23 & 0.12 & 0.33 \\
\hline $\operatorname{misc} 07$ & 62.86 & 60.14 & 54.57 & 60.14 & 60.14 & 60.14 \\
\hline mitre & 0.00 & 1.71 & 1.71 & 1.56 & 1.64 & 1.85 \\
\hline $\mathrm{mkc}$ & 67.21 & 61.54 & 80.93 & 89.77 & 87.67 & 142.90 \\
\hline $\bmod 008$ & 5.24 & 20.73 & 5.54 & 5.15 & 20.94 & 5.54 \\
\hline $\bmod 010$ & 0.39 & 1.48 & 0.77 & 0.45 & 0.77 & 0.77 \\
\hline $\bmod 011$ & 192.95 & 44.80 & 44.80 & 36.80 & 44.80 & 44.80 \\
\hline modglob & 30.10 & 1.78 & 1.78 & 1.78 & 1.63 & 1.78 \\
\hline noswot & 26.47 & 22.86 & 16.22 & 19.44 & 26.47 & 13.16 \\
\hline nw04 & 7.32 & 5.75 & 5.75 & 3.93 & 3.93 & 3.93 \\
\hline p0033 & 28.17 & 20.94 & 18.55 & 18.56 & 18.56 & 18.55 \\
\hline p0201 & 14.65 & 10.89 & 10.89 & 12.03 & 10.89 & 10.89 \\
\hline p0282 & 48.75 & 54.03 & 52.64 & 44.33 & 53.78 & 53.18 \\
\hline p0548 & 93.14 & 97.65 & 97.58 & -- & -- & -- \\
\hline $\mathrm{p} 2756$ & 86.28 & 89.71 & 92.93 & 86.87 & 85.20 & 92.93 \\
\hline pk1 & 100.00 & 100.00 & 100.00 & 100.00 & - & 100.00 \\
\hline pp08a & 72.60 & 78.38 & 78.38 & 78.76 & 77.34 & 78.38 \\
\hline pp08aCUTS & 38.35 & 41.63 & 38.42 & 40.88 & 33.57 & 40.23 \\
\hline qiu & 333.02 & 376.88 & 313.30 & 376.88 & 313.30 & 313.30 \\
\hline qnet1 & 14.44 & 83.49 & 36.03 & 32.65 & 33.95 & 33.95 \\
\hline qnet1_o & 35.20 & 91.88 & 45.58 & 42.47 & 48.00 & 55.74 \\
\hline rentacar & 4.71 & 52.91 & 52.91 & 22.79 & 52.91 & 52.91 \\
\hline rgn & 40.63 & 40.63 & 40.63 & 40.63 & 40.63 & 40.63 \\
\hline rout & 31.05 & 27.61 & 30.44 & 32.78 & 28.63 & 27.19 \\
\hline set1ch & 62.41 & 54.47 & 53.53 & 52.22 & 51.81 & $\mathbf{5 3 . 5 3}$ \\
\hline seymour & 8.16 & 7.37 & 9.60 & 8.84 & 8.84 & 6.30 \\
\hline stein 27 & 31.58 & 31.58 & 31.58 & 27.78 & 31.58 & 31.58 \\
\hline stein 45 & 33.33 & 31.25 & 31.25 & 31.25 & 31.25 & 33.33 \\
\hline swath & 58.95 & 53.54 & 45.36 & 56.83 & 44.92 & 45.40 \\
\hline vpm1 & 25.40 & 32.97 & 35.76 & 29.92 & 32.97 & 35.76 \\
\hline vpm2 & 42.36 & 42.73 & 42.73 & 46.54 & 41.04 & 46.54 \\
\hline \multicolumn{2}{|c|}{$\begin{array}{l}\text { Total \# of better/equal } \\
\text { optimality gap for the } \\
\text { results that are faster/ } \\
\text { equal to CPLEX } 6.5\end{array}$} & $22 / 6$ & $18 / 5$ & $18 / 2$ & $22 / 3$ & $19 / 4$ \\
\hline \multicolumn{7}{|c|}{$\begin{array}{l}\text { Total \# of faster/equal } \\
\text { results compared to }\end{array}$} \\
\hline CPLEX 6.5 & & $44 / 1$ & $38 / 1$ & $34 / 0$ & $35 / 1$ & $37 / 0$ \\
\hline
\end{tabular}

Active Constraint Solver

\begin{tabular}{|c|c|c|}
\hline \multicolumn{3}{|c|}{ Active Constraint Solver } \\
\hline G/DFS & K/DFS & M/DFS \\
\hline
\end{tabular}


Appendix D: Comparison of CPLEX 6.5, Active Constraint Solver G/DFS and

OSLMSLV 3.0 MILP solver for finding the first feasible solution of MULP problems

of MIPLIB 3.0 library

The information in the following table is used to calculate FSR, FSF, FSF', QSR and QSR' of the MILP solvers. For each MILP problem, the number of nodes solved by each solver is shown in the table. The numbers appearing in boldface indicate that the corresponding MILP solver (column) finds the first integer feasible solution faster/equally as fast as the other two solvers for the given MLP problem (row). The optimality gap at the first feasible solution is shown in brackets. The bracketed number appears in boldface if the feasible solution was found faster or equally as fast as the other solvers and the optimality gap at the feasible solution is at least not worst than that of the other solvers. The "--" sign below indicates that no integer feasible solution for that MILP problem is found by the solver within the specified limits and so the optimality gap is unknown.

MILP Problem Name
Number of B\&B nodes solved to find the first feasible solution and the \% optimality gap at the first feasible solution

$\begin{array}{lll}\text { CPLEX 6.5 } & \text { Active Constraint } & \text { OSLMSLV 3.0 } \\ \text { MILP Solver } & \text { Solver G/DFS } & \text { MLP Solver }\end{array}$

\begin{tabular}{llll}
\hline 10teams & $7814(1.29)$ & $661(0.76)$ & $29(2.65)$ \\
air03 & $\mathbb{1}(\mathbf{0 . 0 0 )}$ & $5(8.15)$ & $5(8.52)$ \\
air04 & $\mathbf{8 ( 1 . 4 6 )}$ & $28(6.99)$ & $62(1.47)$ \\
air05 & $35(5.58)$ & $21(3.71)$ & $71(3.59)$ \\
arki001 & $296(0.19)$ & $51714+(-)$ & $194(0.17)$ \\
bell3a & $16(0.78)$ & $23(48.73)$ & $66(5.04)$ \\
bell5 & $62(3.01)$ & $\mathbf{2 0}(3.95)$ & $39(4.44)$ \\
blend2 & $637(25.93)$ & $45(21.54)$ & $280(27.23)$ \\
cap6000 & $915(0.04)$ & $7626+(--)$ & $4886(0.16)$
\end{tabular}


continue...

MILP Problem

Name
Number of $B \& B$ nodes solved to find the first feasible solution and the $\%$ optimality gap at the first feasible solution

\begin{tabular}{|c|c|c|c|}
\hline dano3mip & $46+(--)$ & $46(26.67)$ & $90(21.57)$ \\
\hline danoint & $769(18.52)$ & $1516(29.62)$ & $36(23.61)$ \\
\hline dcmulti & $30(1.80)$ & $39(23.21)$ & $44(2.60)$ \\
\hline dsbmip & $30(8.35)$ & $15(0.00)$ & $37(9.23)$ \\
\hline egout & $14(38.23)$ & $15(41.44)$ & $93(74.29)$ \\
\hline enigma & $5975(0.00)$ & $545(0.00)$ & $3680(0.00)$ \\
\hline fast0507 & $2404(4.89)$ & $43(1.63)$ & $145(6.95)$ \\
\hline fiber & $136(69.64)$ & $36(95.62)$ & $68(73.50)$ \\
\hline fixnet6 & $168(83.40)$ & $70(91.46)$ & $214(81.08)$ \\
\hline flugpl & $65(2.47)$ & $2066+(--)$ & $574(2.43)$ \\
\hline gen & $30(0.25)$ & $19(0.10)$ & $33(0.29)$ \\
\hline gesa2 & $97(1.98)$ & $65(1.27)$ & $91(1.88)$ \\
\hline gesa2_o & $110(2.37)$ & $66(1.34)$ & $143(2.41)$ \\
\hline gesa3 & $58(1.30)$ & $61(1.41)$ & $62(1.36)$ \\
\hline gesa $3 \_0$ & $59(1.31)$ & $55(0.65)$ & $87(3.21)$ \\
\hline $\mathrm{gt} 2$ & $84(86.51)$ & $21(83.44)$ & $267(82.90)$ \\
\hline harp 2 & $392(5.95)$ & $2393+(--)$ & $432(2.04)$ \\
\hline khb05250 & $22(12.66)$ & $18(21.09)$ & $22(11.87)$ \\
\hline $1152 \mathrm{lav}$ & $17(4.91)$ & $29(5.11)$ & $30(5.80)$ \\
\hline 1seu & $146(33.99)$ & $2147+(--)$ & $13(34.43)$ \\
\hline markshare 1 & $27(100.00)$ & $23(100.00)$ & $53(100.00)$ \\
\hline markshare2 & $42(100.00)$ & $66(100.00)$ & $68(100.00)$ \\
\hline $\operatorname{mas} 74$ & $82(38.89)$ & $21(40.40)$ & $41(39.91)$ \\
\hline $\operatorname{mas} 76$ & $81(13.10)$ & $16(12.64)$ & $19(5.62)$ \\
\hline misc03 & $65(58.61)$ & $5(48.10)$ & $80(47.50)$ \\
\hline $\operatorname{misc} 06$ & $20(0.06)$ & $20(0.19)$ & $20(0.11)$ \\
\hline $\operatorname{misc0} 7$ & $282(62.86)$ & $7(60.14)$ & $16(58.44)$ \\
\hline mitre & $279(0.00)$ & $72(1.71)$ & $155231+(--)$ \\
\hline $\mathrm{mkc}$ & $144(67.21)$ & $213(61.54)$ & $2330(45.50)$ \\
\hline $\bmod 008$ & $202(5.24)$ & $7(20.73)$ & $238(29.90)$ \\
\hline $\bmod 010$ & $24(0.39)$ & $23(1.48)$ & $6(0.32)$ \\
\hline $\bmod 011$ & $48(192.95)$ & $17(44.80)$ & $41(21.04)$ \\
\hline modglob & $64(30.10)$ & $32(1.78)$ & $137(3.11)$ \\
\hline noswot & $33(26.47)$ & $55(22.86)$ & $88(16.22)$ \\
\hline nw04 & $468(7.32)$ & $3(5.75)$ & $22(4.85)$ \\
\hline p0033 & $17(28.17)$ & $176(20.94)$ & $100(29.89)$ \\
\hline p0201 & $67(14.65)$ & $13(10.89)$ & $25(13.47)$ \\
\hline p0282 & $22(48.75)$ & $47(54.03)$ & $77(38.41)$ \\
\hline p0548 & $129(93.14)$ & $87(97.65)$ & $335134+(--)$ \\
\hline p2756 & $407(86.28)$ & $27(89.71)$ & $600(81.70)$ \\
\hline pk1 & $42(100.00)$ & $24(100.00)$ & $58(100.00)$ \\
\hline pp08a & $54(72.60)$ & $48(78.38)$ & $80(65.34)$ \\
\hline pp08aCUTS & $52(38.35)$ & $30(41.63)$ & $68(29.46)$ \\
\hline
\end{tabular}


continue...

MILP Problem

Number of B\&B nodes solved to find the first feasible solution and the $\%$

Name

optimality gap at the first feasible solution

$\begin{array}{lll}\text { CPLEX 6.5 } & \text { Active Constraint } & \text { OSLMSLV 3.0 } \\ \text { MILP Solver } & \text { Solver G/DFS } & \text { MLLP Solver }\end{array}$

\begin{tabular}{llll}
\hline qiu & $35(333.02)$ & $\mathbf{2 2 ( 3 7 6 . 8 8 )}$ & $40(563.59)$ \\
qnet1 & $\mathbf{2 2 ( 1 4 . 4 4 )}$ & $54(83.49)$ & $44(12.46)$ \\
qnet1_o & $31(35.20)$ & $71(91.88)$ & $53(28.23)$ \\
rentacar & $14(4.71)$ & $9(52.91)$ & $36(9.98)$ \\
rgn & $58(40.63)$ & $5(40.63)$ & $18(40.63)$ \\
rout & $93(31.05)$ & $\mathbf{6 3 ( 2 7 . 6 1 )}$ & $1457(22.46)$ \\
set1ch & $152(62.41)$ & $\mathbf{9 3 ( 5 4 . 4 7 )}$ & $213(50.20)$ \\
seymour & $131(8.16)$ & $\mathbf{1 1 1 ( 7 . 3 7 )}$ & $253(9.86)$ \\
stein27 & $18(31.58)$ & $16(31.58)$ & $16(31.58)$ \\
stein45 & $30(33.33)$ & $\mathbf{2 9}(31.25)$ & $33(33.33)$ \\
swath & $6206(58.95)$ & $\mathbf{2 7}(\mathbf{5 3 . 5 4 )}$ & $166(38.83)$ \\
vpm1 & $38(25.40)$ & $\mathbf{2 2}(32.97)$ & $38(29.92)$ \\
vpm2 & $435(42.36)$ & $\mathbf{2 9 ( 4 2 . 7 3 )}$ & $76(48.63)$ \\
& & & \\
\hline
\end{tabular}

Total \# of results faster/equal to other solvers (Total \# of results that are faster/equal to other solvers and the optimality gap is better/equal to at least one other solver)

Total \# of comparable results 


\section{Appendix E: Comparison of CPLEX 6.5, Active Constraint Solver G/DFS and \\ OSLMSLV 3.0 MLLP solver for finding the first feasible solution of the MILP problems of FCTP library}

The information in the following table is used to calculate FSR, FSF, FSF', QSR and QSR' of the MILP solvers. For each MILP problem, the number of nodes solved by each solver is shown in the table. The numbers appearing in boldface indicate that the corresponding MILP solver (column) finds the first integer feasible solution faster/equally as fast as the other two solvers for the given MILP problem (row). The optimality gap at the first feasible solution is shown in brackets. The bracketed number appears in boldface if the feasible solution was found faster or equally as fast as the other solvers and the optimality gap at the feasible solution is at least not worst than that of the other solvers.

MILP Problem Name
Number of $B \& B$ nodes solved to find the first feasible solution and the $\%$ optimality gap at the first feasible solution

$\begin{array}{lll}\text { CPLEX 6.5 } & \text { Active Constraint } & \text { OSLMSLV 3.0 } \\ \text { MLP Solver } & \text { Solver G/DFS } & \text { MLLP Solver }\end{array}$

$\begin{array}{llll}\operatorname{ran} 10 \times 10 \mathrm{a} & 39(39.83) & \mathbf{1 6}(\mathbf{3 1 . 5 4 )} & 47(31.77) \\ \operatorname{ran} 10 \times 10 \mathrm{~b} & 55(40.92) & \mathbf{1 5}(\mathbf{3 3 . 7 5 )} & 46(28.59) \\ \operatorname{ran} 10 \times 10 \mathrm{c} & 49(31.24) & \mathbf{1 5}(\mathbf{2 1 . 1 4 )} & 72(34.08) \\ \operatorname{ran} 10 \times 12 & 64(34.62) & \mathbf{1 7}(\mathbf{2 9 . 3 3 )} & 46(30.92) \\ \operatorname{ran} 10 \times 26 & 130(29.67) & \mathbf{2 8}(\mathbf{2 5 . 2 2 )} & 150(23.41) \\ \operatorname{ran} 12 \times 12 & 58(43.15) & \mathbf{2 2 ( 3 1 . 9 2 )} & 73(34.67) \\ \operatorname{ran} 12 \times 21 & 103(34.26) & \mathbf{2 6}(\mathbf{2 3 . 8 6 )} & 126(25.83) \\ \operatorname{ran} 13 \times 13 & 79(36.73) & \mathbf{2 0}(\mathbf{2 4 . 5 9 )} & 66(27.77) \\ \operatorname{ran} 14 \times 18 & 166(41.31) & \mathbf{3 6}(39.43) & 151(34.13) \\ \operatorname{ran} 16 \times 16 & 94(39.07) & \mathbf{2 6}(\mathbf{2 8 . 4 6 )} & 89(27.79) \\ \operatorname{ran} 17 \times 17 & 131(44.08) & \mathbf{3 2}(25.90) & 147(29.96) \\ \operatorname{ran} 4 \times 64 & 85(1.66) & 13(3.66) & \mathbf{1 0}(\mathbf{1 . 7 3 )} \\ \operatorname{ran} 6 \times 43 & 54(3.82) & \mathbf{2 1}(10.17) & 90(8.53) \\ \operatorname{ran} 8 \times 32 & 82(20.16) & \mathbf{2 6}(\mathbf{1 8 . 6 4 )} & 97(19.91)\end{array}$


to other solvers (Total \# of results that are faster/equal to other solvers and the optimality gap is better/equal to at least one other solver)

Total \# of comparable results 
Appendix F: Comparison of CPLEX 6.5, Active Constraint Solver G/DFS and

OSLMSLV 3.0 MILP solver for finding the first feasible solution of the MILP

problems of the MIP Benchmark library

The information in the following table is used to calculate FSR, FSF, FSF', QSR and QSR' of the MILP solvers. For each MILP problem, the number of nodes solved by each solver is shown in the table. The numbers appearing in boldface indicate that the corresponding MILP solver (column) finds the first integer feasible solution faster/equally as fast as the other two solvers for the given MILP problem (row). The optimality gap at the first feasible solution is shown in brackets. The bracketed number appears in boldface if the feasible solution was found faster or equal as fast as the other solvers and the optimality gap at the feasible solution is at least not worst than that of the other solvers.

\begin{tabular}{|c|c|c|c|}
\hline \multirow[t]{2}{*}{$\begin{array}{l}\text { MILP Problem } \\
\text { Name }\end{array}$} & \multirow[b]{2}{*}{$\begin{array}{l}\text { CPLEX } 6.5 \\
\text { MILP Solver }\end{array}$} & \multirow[b]{2}{*}{$\begin{array}{l}\text { Active Constraint } \\
\text { Solver } G / D F S\end{array}$} & \multirow[b]{2}{*}{$\begin{array}{l}\text { OSLMSLV } 3.0 \\
\text { MILP Solver }\end{array}$} \\
\hline & & & \\
\hline $\begin{array}{l}\text { bc } \\
\text { binkar10_1 } \\
\text { eilD76 } \\
\text { haprp } \\
\text { irp } \\
\text { mas284 } \\
\text { prod1 } \\
\text { rlp2 }\end{array}$ & $\begin{array}{l}420(96.42) \\
111(9.68) \\
341(30.93) \\
1491(0.51) \\
255(0.68) \\
147(15.17) \\
59(67.35) \\
129(48.77)\end{array}$ & $\begin{array}{l}10(91.30) \\
154(15.70) \\
6(38.92) \\
1041(0.70) \\
3(0.59) \\
9(8.29) \\
36(74.47) \\
22(46.26)\end{array}$ & $\begin{array}{l}83(92.15) \\
121(6.65) \\
508(55.74) \\
132(0.26) \\
14(11.93) \\
8(10.09) \\
88(106.38) \\
103(48.94)\end{array}$ \\
\hline $\begin{array}{l}\text { Total \# of results faster/equal } \\
\text { to other solvers (Total \# of } \\
\text { results that are faster/equal } \\
\text { to other solvers and the } \\
\text { optimality gap is better/equal } \\
\text { to at least one other solver) }\end{array}$ & $1(1)$ & $5(5)$ & $2(2)$ \\
\hline Total \# of comparable results & 8 & 8 & 8 \\
\hline
\end{tabular}




\section{Appendix G: Comparison of CPLEX 6.5, Active Constraint Solver G/DFS and \\ OSLMSLV 3.0 MILP solver for finding the first feasible solution of the MILP problems of the MP Test Model library}

The information in the following table is used to calculate FSR, FSF, FSF', QSR and QSR' of the MILP solvers. For each MLP problem, the number of nodes solved by each solver is shown in the table. The numbers appearing in boldface indicate that the corresponding MILP solver (column) finds the first integer feasible solution faster/equally as fast as the other two solvers for the given MILP problem (row). The optimality gap at the first feasible solution is shown in brackets. The bracketed number appears in boldface if the feasible solution was found faster or equally as fast as the other solvers and the optimality gap at the feasible solution is at least not worst than that of the other solvers.

MILP Problem Name
Number of B\&B nodes solved to find the first feasible solution and the $\%$ optimality gap at the first feasible solution

$\begin{array}{lll}\text { CPLEX 6.5 } & \text { Active Constraint } & \text { OSLMSLV 3.0 } \\ \text { MILP Solver } & \text { Solver G/DFS } & \text { MILP Solver }\end{array}$

$\begin{array}{llll}\text { bc1 } & 154(89.64) & \mathbf{4 ( 7 7 . 1 7 )} & 13(82.26) \\ \text { bienst1 } & \mathbf{9 ( 8 3 . 0 9 )} & \mathbf{9 ( 8 3 . 4 3 )} & 27(85.70) \\ \text { bienst2 } & \mathbf{1 1 ( 8 1 . 6 8 )} & \mathbf{1 1}(84.37) & 34(82.24) \\ \text { dano3_3 } & 14(0.33) & \mathbf{6 ( 0 . 2 5 )} & 11(0.03) \\ \text { dano3_4 } & 13(0.43) & \mathbf{7}(\mathbf{0 . 3 3 )} & 16(0.24) \\ \text { dano3_5 } & 15(0.59) & \mathbf{1 0}(0.59) & 13(0.37) \\ \text { mkc1 } & 54(10.42) & 75(5.21) & 378(4.64) \\ \text { neos1 } & 807(83.03) & \mathbf{5 9 ( 8 2 . 5 0 )} & 169(82.50) \\ \text { neos2 } & 705(1047.53) & 11866(488.20) & \mathbf{5 8 1}(\mathbf{5 0 4 . 6 3 )} \\ \text { neos3 } & 748(1583.00) & 51093(634.38) & \mathbf{7 2 6}(595.90) \\ \text { nug08 } & 387(7.50) & \mathbf{2}(8.33) & 6(4.91) \\ \text { qap10 } & 758(11.55) & \mathbf{2 ( 7 . 6 2 )} & 23(9.13) \\ \text { seymour1 } & 44(2.87) & 47(3.24) & 58(2.41) \\ \text { swath1 } & 449(48.77) & \mathbf{8 ( 2 3 . 6 8 )} & 104(19.11) \\ \text { swath2 } & 919(54.14) & \mathbf{9 ( 2 6 . 1 3 )} & 98(21.34)\end{array}$


Total \# of results faster/equal to other solvers (Total \# of results that are faster/equal to other solvers and the optimality gap is better/equal to at least one other solver)

Total \# of comparable results 
Appendix H: Comparison of the proposed Active Constraint Solvers in finding the optimal/final solution of the MILP problems of MIPLIB 3.0 library

The information in the following table is used to calculate the OSR of the MILP solvers. For each MILP problem, the number of nodes solved by each solver is shown in the table. The numbers appearing in boldface indicate that the corresponding Active Constraint solver (column) finds the optimal solution faster/equally as fast as the CPLEX 6.5 MLP solver for the given MILP problem (row). If the limit (e.g time or node limit) is reached before the optimal solution is found and the results are comparable, then the optimality gaps are compared in order to decide if the Active Constraint Solver found a better result compared to CPLEX 6.5. The optimality gap of the solution that is found by the solver is shown in brackets. A "_" sign indicates that the problem was not selected for solving by that solver. The "--" sign below indicates that no integer feasible solution for that MILP problem is found by the solver within the specified limits and so the optimality gap is unknown.

$\begin{aligned} & \text { MILP Problem } \\
& \text { Name }\end{aligned}$
$\begin{array}{lll}\text { Number of B\&B nodes solved to find optimal/final solution (\% Optimality gap at } \\
\text { the final solution) }\end{array}$
\begin{tabular}{llll} 
CPLEX 6.5 & Active Constraint & Active Constraint \\
& & Solver O & Solver P \\
\hline & & & \\
10teams & $139160+(0.43)$ & $91160+(0.76)$ & $1288(0.00)$ \\
air03 & $1(0.00)$ & $1+(-)$ & $1+(--)$ \\
air04 & $237(0.00)$ & $237+(5.26)$ & $237+(0.46)$ \\
air05 & $586(0.00)$ & $586+(2.77)$ & $586+(2.19)$ \\
arki001 & $987624+(0.04)$ & - & $101403+(-)$ \\
bel13a & $17037(0.00)$ & $17037+(0.44)$ & $17037+(0.09)$ \\
bel15 & $5424(0.00)$ & $5424+(0.11)$ & $2921(0.00)$ \\
blend2 & $2461(0.00)$ & $2461+(0.50)$ & $2461+(9.56)$
\end{tabular}


continue...

MLP Problem

Name
Number of $B \& B$ nodes solved to find optimal/final solution

CPLEX 6.5

MILP Solver
Active Constraint

Solver O
Active Constraint

Solver $\mathrm{P}$

\begin{tabular}{|c|c|c|c|}
\hline cap 6000 & $1428(0.00)$ & $1428+(--)$ & $1428+(--)$ \\
\hline dano3mip & $46+(--)$ & $46+(26.67)$ & $46+(26.67)$ \\
\hline danoint & $177538+(8.03)$ & $177538+(4.63)$ & $177538+(4.42)$ \\
\hline dcmulti & $905(0.00)$ & $905+(4.41)$ & $905+(0.05)$ \\
\hline dsbmip & $77(0.00)$ & $15(0.00)$ & $\mathbb{1 5}(0.00)$ \\
\hline egout & $1487(0.00)$ & $1487+(14.97)$ & $1477(0.00)$ \\
\hline enigma & $5975(0.00)$ & $545(0.00)$ & $545(0.00)$ \\
\hline fast0507 & $4791+(4.51)$ & $1403+(1.55)$ & $4791+(1.21)$ \\
\hline fiber & $62935(0.00)$ & $62935+(81.84)$ & $56181(0.00)$ \\
\hline fixnet6 & $6800302+(32.74)$ & $632625+(87.53)$ & $6800302+(32.95)$ \\
\hline flugpl & $316(0.00)$ & $316+(--)$ & $316+(--)$ \\
\hline gen & $274(0.00)$ & $274+(0.05)$ & $274+(0.05)$ \\
\hline gesa2 & $140107(0.00)$ & $110133+(0.68)$ & $140107+(0.03)$ \\
\hline gesa2_o & $99861(0.00)$ & $99861+(0.92)$ & $99861+(0.14)$ \\
\hline gesa3 & $1128(0.00)$ & $1128+(1.19)$ & $1128+(0.93)$ \\
\hline gesa3_o & $2580(0.00)$ & $2580+(0.29)$ & $1719(0.00)$ \\
\hline $\mathrm{gt} 2$ & $549(0.00)$ & $549+(83.20)$ & $549+(23.52)$ \\
\hline harp2 & $1554972+(0.20)$ & - & $1554972+(0.19)$ \\
\hline khb05250 & $1474(0.00)$ & $1474+(4.19)$ & $1447(0.00)$ \\
\hline $11521 \mathrm{lav}$ & $362(0.00)$ & $362+(2.28)$ & $362+(0.35)$ \\
\hline lseu & $17196(0.00)$ & $17196+(28.48)$ & $17196+(7.07)$ \\
\hline markshare1 & $52386302+(100.00)$ & - & $36495491+(100.00)$ \\
\hline markshare2 & $21921986+(100.00)$ & - & $20543432+(100.00)$ \\
\hline $\operatorname{mas} 74$ & $6271495+(1.55)$ & - & $6271495+(0.79)$ \\
\hline $\operatorname{mas} 76$ & $593840(0.00)$ & - & $447481(0.00)$ \\
\hline $\operatorname{misc} 03$ & $729(0.00)$ & $729+(9.71)$ & $729+(4.09)$ \\
\hline $\operatorname{misc06}$ & $52(0.00)$ & $52+(0.17)$ & $52+(0.16)$ \\
\hline misc07 & $63400(0.00)$ & $63400+(4.51)$ & $63400+(4.09)$ \\
\hline mitre & $279(0.00)$ & $279+(1.70)$ & $279+(1.65)$ \\
\hline mkc & $394630+(16.20)$ & $15554+(61.54)$ & $394630+(22.85)$ \\
\hline $\bmod 008$ & $5524(0.00)$ & $5524+(2.00)$ & $5524+(2.57)$ \\
\hline $\bmod 010$ & $58(0.00)$ & $58+(0.88)$ & $58+(0.42)$ \\
\hline $\bmod 011$ & $33039(0.00)$ & $7203+(34.10)$ & $28245(0.00)$ \\
\hline modglob & $4302207(0.00)$ & - & $4302207+(0.09)$ \\
\hline noswot & $13735472+(4.88)$ & - & $13735472+(4.88)$ \\
\hline nw04 & $807(0.00)$ & $807+(1.91)$ & $271(0.00)$ \\
\hline p0033 & $262(0.00)$ & $262+(20.89)$ & $262+(16.66)$ \\
\hline p0201 & $595(0.00)$ & $595+(3.24)$ & $595+(1.79)$ \\
\hline p0282 & $265(0.00)$ & $265+(53.41)$ & $265+(0.78)$ \\
\hline p0548 & $6620(0.00)$ & $6620+(97.63)$ & $6620+(27.92)$ \\
\hline $\mathrm{p} 2756$ & $990112+(4.65)$ & - & $990112+(5.01)$ \\
\hline $\mathrm{pk} 1$ & $354518(0.00)$ & - & $311559(0.00)$ \\
\hline pp08a & $11043291+(14.39)$ & - & $10706894+(16.25)$ \\
\hline pp08aCUTS & $3784440(0.00)$ & - & $3137205(0.00)$ \\
\hline
\end{tabular}


continue...

MILP Problem Number of B\&B nodes solved to find optimal/final solution

Name

$\begin{array}{lll}\text { CPLEX 6.5 } & \text { Active Constraint } & \text { Active Constraint } \\ \text { MILP Solver } & \text { Solver O } & \text { Solver P }\end{array}$

\begin{tabular}{llll}
\hline qiu & $19227(0.00)$ & $19227+(208.88)$ & $19227+(0.05)$ \\
quet1 & $116(0.00)$ & $116+(41.48)$ & $116+(14.24)$ \\
quet1_o & $448(0.00)$ & $448+(4.26)$ & $448+(13.71)$ \\
rentacar & $24(0.00)$ & $24+(52.06)$ & $24+(14.36)$ \\
rgn & $2702(0.00)$ & $2702+(4.38)$ & $\mathbf{2 5 4 5 ( 0 . 0 0 )}$ \\
rout & $345690(0.00)$ & - & $345690+(3.92)$ \\
set1ch & $8099339+(32.16)$ & - & $\mathbf{7 9 5 3 4 2 8 + ( 2 9 . 1 2 )}$ \\
seymour & $7941+(6.87)$ & - & $\mathbf{7 9 4 1 + ( 6 . 0 7 )}$ \\
stein27 & $3850(0.00)$ & $\mathbf{2 6 3 9 ( 0 . 0 0 )}$ & $\mathbf{3 3 7 2}(\mathbf{0 . 0 0})$ \\
stein45 & $60223(0.00)$ & $\mathbf{3 9 1 4 9}(0.00)$ & $\mathbf{5 5 4 2 9 ( 0 . 0 0 )}$ \\
swath & $529894+(57.72)$ & - & $\mathbf{5 2 9 8 9 4 + ( 5 0 . 4 0 )}$ \\
vpm1 & $168829(0.00)$ & - & $\mathbf{1 6 8 8 2 9 + ( 6 . 8 3 )}$ \\
vpm2 & $348984(0.00)$ & - & $348984+(3.47)$ \\
& & & \\
\hline
\end{tabular}

Total \# of results faster/equal to

$7 / 0$

$24 / 1$

CPLEX 6.5 solver

Total \# of results comparable to

42

61 that of CPLEX 6.5 solver 


\section{Appendix I: Information on the Software Prototype Program}

The following figure shows the Graphical User Interface (GUI) of the C++ prototype program that compares the effectiveness of the Active Constraint Solvers using particular branching variable selection and node selection schemes with the CPLEX 6.5 MILP solver. This program solves the MLP problems using both solvers and compares their results. The GUI allows specification of the directory containing the MILP problems in MPS format, the directories where the results are to be stored and various MILP solver settings. The prototype has extra features that were used in the experimental stage which are not described in this thesis, hence some of the GUI options are irrelevant to this thesis. The following are the various options/selections allowed in the GUI that are relevant to this thesis.

- Mps Dir: The directory containing the MPS files representing the MILP problems to be solved.

- Cplex Dir: The directory where the results of the CPLEX 6.5 MILP solver are stored.

- Result Dir: The directory where the results of the Active Constraint Solver are stored. The report file that compares the results is also stored here.

- MLP Solving Parameters Control: The presolving, aggregation, node heuristics and cut generation parameter settings. If selected then the corresponding feature of the CPLEX 6.5 MILP solver is turned on. Since the Active Constraint Solver is also based on the CPLEX 6.5 MILP solver, the settings have the same effect on it. 
- Solving limits: This is used to specify limits on solving time (in seconds), the number of solved $B \& B$ nodes, the maximum number of feasible solutions, the memory used by the B\&B tree (in Mbytes) and the node file size limit (in Mbytes) for both solvers. A value of -1 indicates that no limit has been set.

- Node selection strategy: To select the node selection scheme that is to be used by the Active Constraint Solver.

- Branch selection strategy: To select the branching variable selection scheme that is to be used by the Active Constraint Solver.

- Most Impact on Single Active Cons: To select the branching variable that has the maximum weight in a single active constraint instead of maximizing the sum of its weight in all active constraint.

- Logging: Controls CPLEX logging and its frequency. An internal log contains details on the runtime behavior of the Active Constraint Solver.

- Disable After First Feas.: Used for optimality tests. If selected then the Active Constraint Solver is continued up to the first feasible solution and then CPLEX default branching variable selection and node selection strategies are used. 




Figure 10: Graphical User Interface (GUI) of the prototype program 